\title{
Effects Of Commercial Formulations Of Glyphosate On Saccharomyces Cerevisiae
}

Apoorva Ravi Shankar

West Virginia University, apravishankar@mix.wvu.edu

Follow this and additional works at: https://researchrepository.wvu.edu/etd

Part of the Biology Commons, Genomics Commons, and the Molecular Genetics Commons

\section{Recommended Citation}

Ravi Shankar, Apoorva, "Effects Of Commercial Formulations Of Glyphosate On Saccharomyces

Cerevisiae" (2019). Graduate Theses, Dissertations, and Problem Reports. 7458.

https://researchrepository.wvu.edu/etd/7458

This Dissertation is protected by copyright and/or related rights. It has been brought to you by the The Research Repository @ WVU with permission from the rights-holder(s). You are free to use this Dissertation in any way that is permitted by the copyright and related rights legislation that applies to your use. For other uses you must obtain permission from the rights-holder(s) directly, unless additional rights are indicated by a Creative Commons license in the record and/ or on the work itself. This Dissertation has been accepted for inclusion in WVU Graduate Theses, Dissertations, and Problem Reports collection by an authorized administrator of The Research Repository @ WVU.

For more information, please contact researchrepository@mail.wvu.edu. 


\title{
Apoorva Ravi Shankar
}

\author{
Dissertation submitted to the Eberly College of Arts and Sciences \\ at West Virginia University \\ in partial fulfillment of the requirements \\ for the degree of \\ Doctor of Philosophy \\ in \\ Biology
}

Jennifer Gallagher, Ph.D., Chair

Stephen DiFazio, Ph.D.

Timothy Driscoll, Ph.D.

Jennifer Hawkins, Ph.D.

Daniel Panaccione, Ph.D.

Department of Biology

Morgantown, West Virginia

2019

Keywords: Saccharomyces cerevisiae, glyphosate-based herbicides, additives, transport, cell wall, mitochondria

Copyright 2019 Apoorva Ravi Shankar 


\section{ABSTRACT \\ Effects of commercial formulations of glyphosate on Saccharomyces cerevisiae Apoorva Ravi Shankar}

Commercial formulations of glyphosate are among the most extensively used herbicides around the world. The active ingredient, glyphosate, targets the aromatic amino acid pathway. This pathway is absent in mammals, resulting in low toxicity. Different formulations contain varying adjuvants and surfactants, whose synergistic effects are yet to be extensively studied at the cellular level. In this study, I tested multiple commercial formulations that showed a variation in growth phenotype among different yeast strains. To gain a better understanding of response and resistance mechanisms at the genome and transcriptome level, I carried out an in-lab evolution study, along with a transcriptome analysis on exposure to a commercial formulation of glyphosate. Overall, my findings helped identify routes of transport of glyphosate in and out of the cell. The additives in these herbicides were found to have effects on the cell wall, cell cycle regulation, transposable elements, and mitochondrial function. 


\section{ACKNOWLEDGEMENTS}

First and foremost, I would like to thank my advisor, Dr. Jennifer Gallagher, for being incredibly supportive for the past five years. Throughout my PhD, she provided insightful suggestions and gave me the freedom to steer my project in the direction I desired. As much knowledge as she has imparted, she has also been instrumental in helping me be a better version of myself. I have learned to stand up for

myself, work hard and have a voice, and for that I owe it to her completely. I want to thank her for being the advisor who pushed me to be better and believed in me even when I didn't believe I could do it.

I am also grateful to my committee members, Dr. DiFazio, Dr. Driscoll, Dr. Hawkins and Dr. Panaccione for readily offering their time and guidance throughout the course of my PhD.

My sincere thanks to Dr. K Muniyappa for giving me an opportunity to be a part of his lab as a technician, as that was the first step towards pursuing my PhD

I am so thankful to Michael Ayers for being the best lab mate and friend anyone could ever ask for. A heartfelt thank you to Dhanushya Ramachandran for being like an elder sister to me, and being there to support and guide me along the way. I thank Melissa Lehrer for being a wonderful friend, who was there to celebrate the happy times, and also let me vent when I needed it. I would like to thank all the past and present members of the Gallagher lab for making it a great working environment. A special thanks to Amaury Pupo for being so patient and helping me with my computational analysis.

I would like to thank Pragya Srivastava for being the best roommate ever. I would also like to express my heartfelt gratitude to Vikas Agrawal, Srikant Gattu, Sobhit Singh and Swathi Reddy Shetty for being my Morgantown family. This journey would have been much harder without them. 
I would like to take this opportunity to express my gratitude to Dr. and Mrs. Nath for welcoming me into their home when I moved to Morgantown and being a constant support. I am extremely grateful to Dr. \& Mrs. Gopala Krishna who accepted me into their family and have been so supportive.

My time in Morgantown would not have been this fun, if not for the WVU Latin Dance Club. A special thanks to Demian Gaxiola and Marriah Ellington for making it happen and giving me a place to de-stress every week.

I would not have followed my dreams, applied to a PhD program and completed it, without Shilpa Poovaiah, who was always there to listen to me when I was excited about a new research idea and cheer me on and believe in me even when everything failed. I thank Jasmin Thomas for being someone I could always rely on for support; along with Akankhya Baruah, Caleb Rajkumar, Vishal Manay, Tejeshvi Lokesh, Ranjit Subburam, Melwin Reuben and all my friends back home who loved and supported me throughout this journey.

I thank my family, all my aunts, uncles and cousins who called to check on me and were always supportive and happy for me. There will always be a special place in my heart for all my grandparents who showered me with unconditional love and were always so proud.

Last but not the least, I would like to thank my parents for being the most amazing parents, and an inspiration to me throughout my life. I can't thank them enough for their endless support. I thank them for letting me follow my dreams and being there at every step along the way. They made me the person I am today. 


\section{DEDICATION}

This dissertation is dedicated to my parents

OH. \& Ravi Shankar

and

Mrs. Aundathi Ravishankar

For their endless love, support, and encouragement 


\section{TABLE OF CONTENTS}

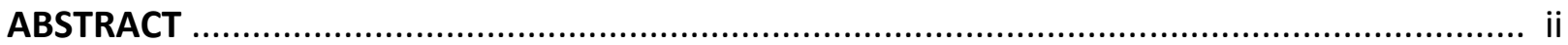

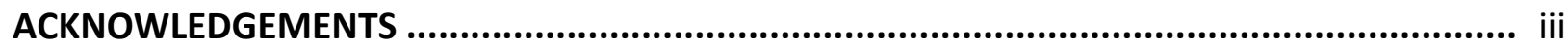

TABLE OF CONTENTS ….................................................................................................... vi

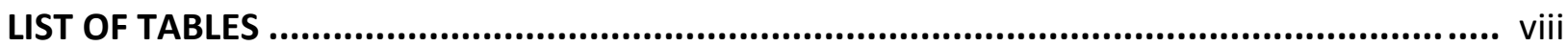

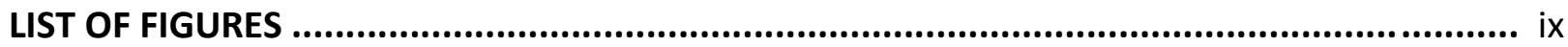

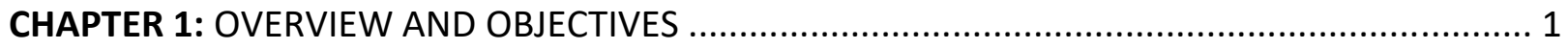

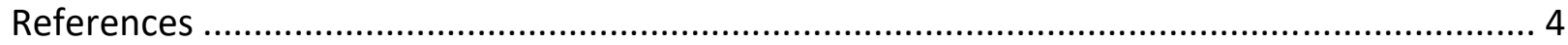

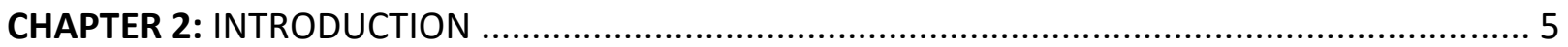

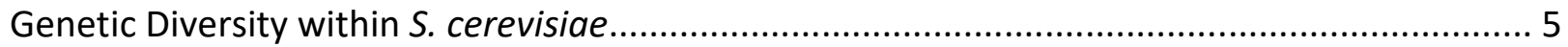

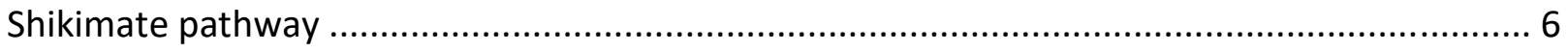

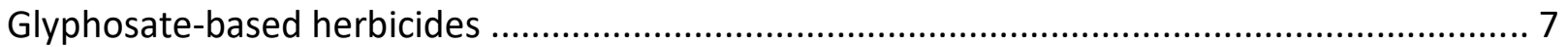

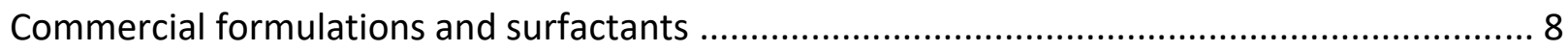

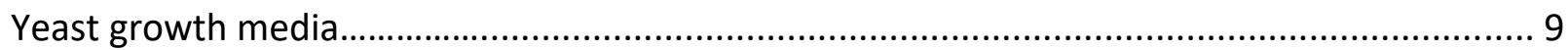

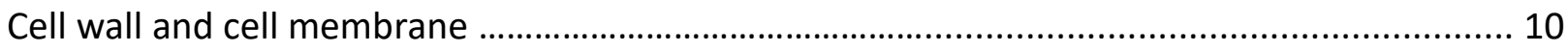

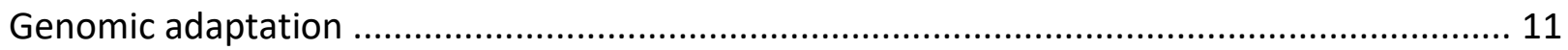

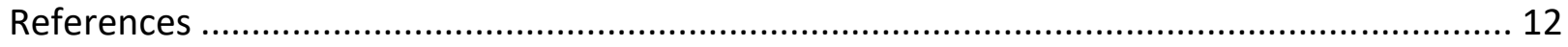

CHAPTER 3: GENETIC VARIATION IN DIP5, AN AMINO ACID PERMEASE, AND PDR5, A MULTIPLE DRUG TRANSPORTER, REGULATES GLYPHOSATE RESISTANCE IN S. CEREVISIAE ...................... 17

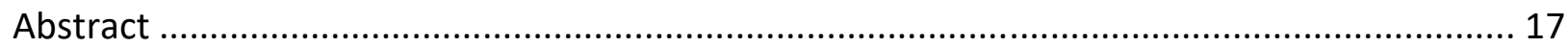

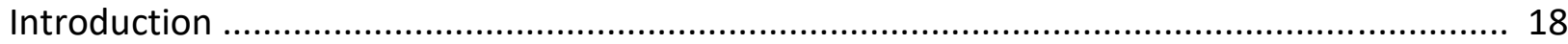

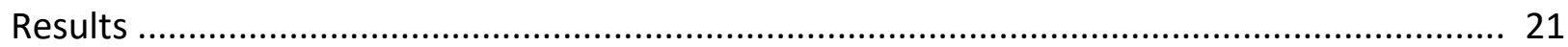

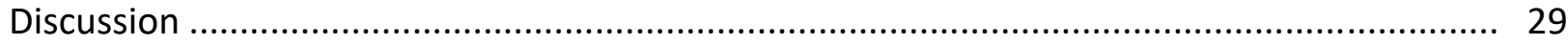

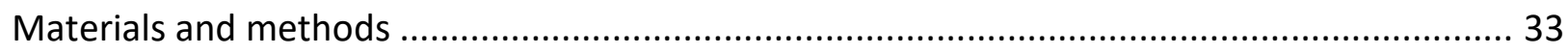

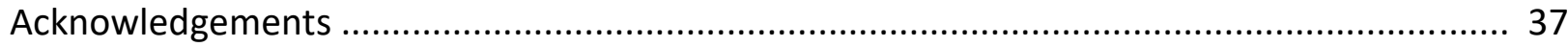

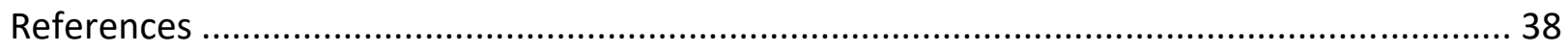

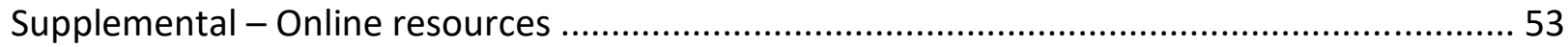

CHAPTER 4: THICKENING OF THE CELL WALL INCREASES RESISTANCE OF S. CEREVISIAE TO

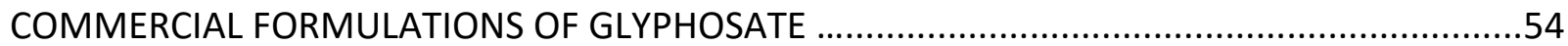




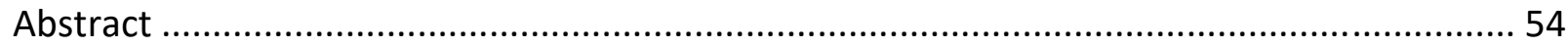

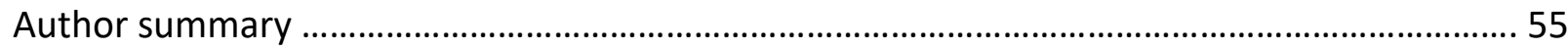

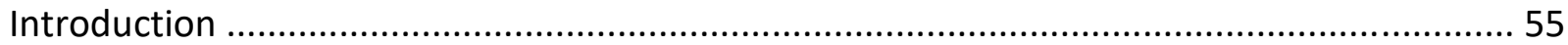

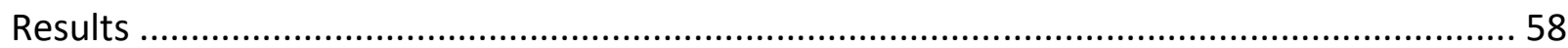

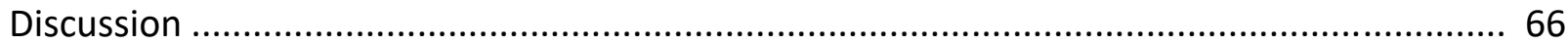

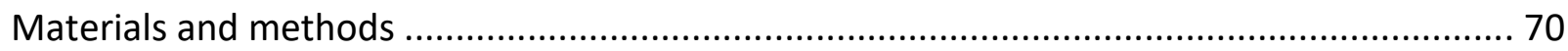

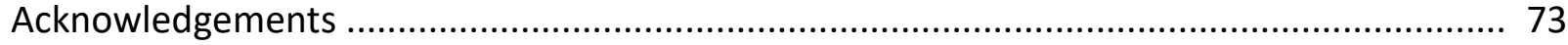

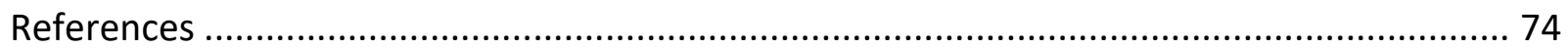

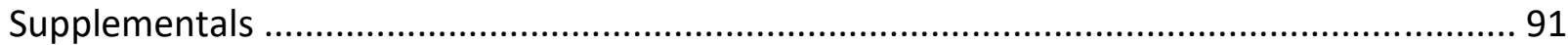

CHAPTER 5: MITOCHONDRIAL METABOLISM IS CRUCIAL IN RESPONSE AND RESISTANCE MECHANISMS OF SACCHAROMYCES CEREVISIAE ON EXPOSURE TO A GLYPHOSATE-BASED

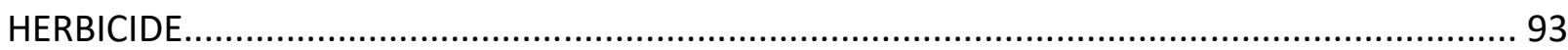

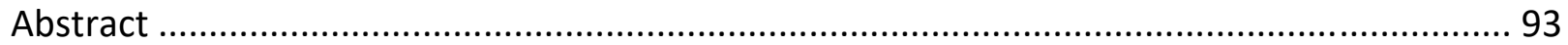

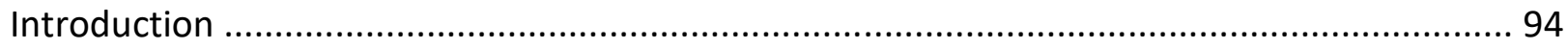

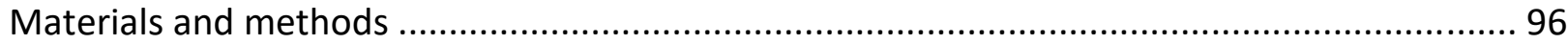

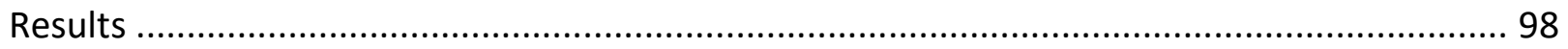

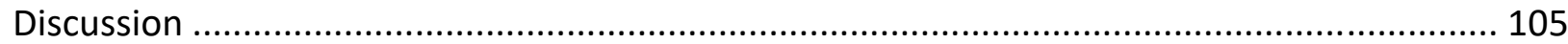

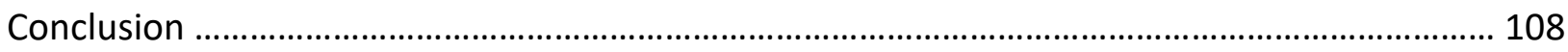

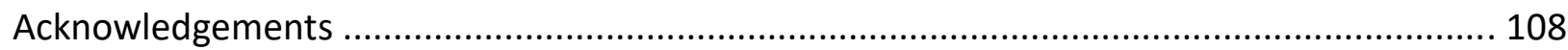

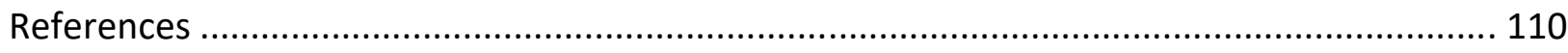

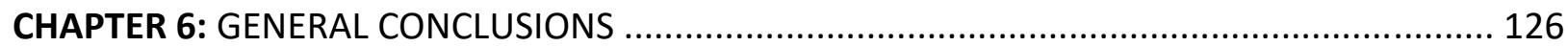

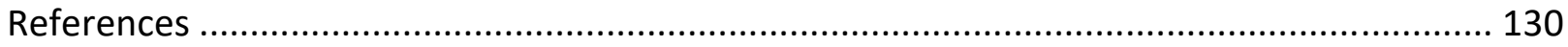




\section{LIST OF TABLES}

List of yeast used in this study

QTL mapping between S288c and YJM789 on 1\% glyphosate on YPD 53

QTL mapping between S288c and YJM789 on 0.15\% glyphosate on WYF 53

QTL mapping between S288c and YJM789 on 0.15\% glyphosate on YM 53

Regions with CNVs flanked by fragile sites in the ILEs $\quad 80$

$\begin{array}{ll}\text { Strain List } & 92\end{array}$

List of strains evolved in respective conditions $\quad 92$

List of genes and number of samples containing SNPs in the ILEs 92

List of genes that underwent Copy Number variation in the ILEs 92

RNAseq analysis of GSY147 with and without Credit41 treatment 92

RNAseq analysis of GSY147 supplemented with WYF, with \& without Credit41 treatment 92

RNAseq analysis of RM11 with and without Credit41 treatment 92

RNAseq analysis of RM11 supplemented with WYF, with \& without Credit41 treatment 92

$\begin{array}{ll}\text { Mitochondrial proteome genes that underwent CNV } & 116\end{array}$ 


\section{LIST OF FIGURES}

$\begin{array}{ll}\text { Aromatic amino acid biosynthetic pathway } & 15\end{array}$

Saccharomyces cerevisiae cell wall and cell membrane structure 16

Genetic variation effects growth inhibition by glyphosate 43

Contribution of the genetic variation within Aro1 to glyphosate resistance $\quad 44$

Different responses of aro1 $\Delta$ yeast on media supplemented with different aromatic Metabolites $\quad 45$

Genetic linkage analysis of glyphosate sensitivity in glyphosate on rich media $\quad 46$

Genetic Linkage analysis of glyphosate sensitivity in glyphosate on minimal media with and without aromatic amino acids

Regulation of DIP5 by glyphosate and aspartic acid $\quad 48$

Aro1 is a pentafunctional enzyme in the shikimate pathway 49

$\mathrm{N}$-rooted phylogenetic tree of Pdr5 protein from genetically different yeast $\quad 50$

$\mathrm{N}$-rooted phylogenetic tree of Dip5 protein from genetically different yeast $\quad 51$

Ectopic expression of PDR5 alleles in S288c and YJM789 with and without ARO1 52

Contribution of genetic variation of different yeast strains to glyphosate resistance 81

Selection of strains resistant to commercial glyphosate formula, Credit41 by In-lab evolutions (ILEs) $\quad 82$

Whole-genome analysis of In-lab evolutions (ILEs) 83

Gene expression analysis of S288c and RM11 on exposure to glyphosate (Credit41) in minimal media $\quad 84$

Cell cycle distribution on treatment with glyphosate and commercial glyphosate formulation $\quad 85$

Growth assay on exposure to calcofluor white and glyphosate-based herbicides 86

Alignment of ILE strains containing mutations in DIP5 87

Cross resistance was conferred in strains evolved in minimal media and WYF $\quad 88$

Sample to sample distance estimation using DESeq2 89 
Flow cytometry of S288c strains show arrest in G1 phase of the cell cycle on treatment with $\mathrm{Cr} 41$

Pure glyphosate inhibits growth to a greater extent than $\mathrm{Cr} 41$ only in strains closely related to $\mathrm{S} 288 \mathrm{c}$

Genetic linkage analysis of glyphosate sensitivity in glyphosate (Credit41) in minimal media

Growth of RM11 and S288c (BY4742) haploid mutants on exposure to Credit 41 (Cr41) 118

Growth of RM11 double knockouts on exposure to Credit 41 (Cr41)

Gene expression analysis of RM11 on exposure to glyphosate $(\mathrm{Cr} 41)$ in minimal media

Analysis of genes affected in the in-lab evolutions and their relatedness to the mitochondria

Change in metal amounts on glyphosate exposure

Growth of RM11 knockouts on exposure to Credit 41 (Cr41) and pure glyphosate (PG) 


\section{CHAPTER 1: OBJECTIVES AND OVERVIEW}

Glyphosate is one of the most commonly used herbicides in the world due to its versatility, ability to control a wide spectrum of weeds, and its minimal toxicity to mammals. Glyphosate targets the shikimate pathway that is absent in mammals, making it less toxic in comparison to most other herbicides (Powles and Preston 2006). However, the increased use of glyphosate is correlated with increased exposure to humans, as measured through human uptake (Mills et al. 2017a). Glyphosate (Nphosphonomethylglycine) the active ingredient, acts as an effective herbicide by targeting the 5enolpyruvylshikimate-3-phosphate synthase (EPSPS) enzyme in the shikimate pathway. This enzyme is essential in plants, fungi, and bacteria because it has a vital role in the aromatic amino acid (phenylalanine, threonine, and tyrosine) biosynthesis pathway. Organisms can undergo adaptations for survival when they come in contact with environmental stressors. Widespread exposure of organisms to glyphosate over extended periods of time gave rise to plants that are resistant to glyphosate-based herbicides (Schütte et al. 2017). Glyphosate resistant organisms have evolved in nature due to exposure, as well as been developed in laboratories via artificial mutagenesis in-vitro (Healy-Fried et al. 2007). Sitedirected mutagenesis can be used to induce specific point mutations in the EPSPS gene, leading to glyphosate resistance (Amrhein et al. 1983); the over-expression of EPSPS in the system also results in glyphosate resistance (Baerson et al. 2002).

Most of the studies carried out to analyze effects of herbicides on organisms use the pure form of the active ingredient, rather than using the commercially available formulations. These commercial formulations are comprised of the active ingredient along with other additives, such as detergents. Though the canonical target of glyphosate and the affected pathway has been studied in great detail, there is still a gap in knowledge when it comes to the commercial formulations that are used extensively 
throughout the world. It is only recently that the importance of studying the effects of these commercial formulations, has come to light. Multiple insects and animals are exposed to these herbicides on a daily basis. Although they do not contain the shikimate pathway, we are unaware of the effects these herbicides have on other pathways. Hence, it is important to identify the changes these products bring about as a whole, at the cellular level.

The purpose of my research was to use the extensive genetic diversity across different yeast strains to gain a better understanding of how organisms adapt to different forms of stress in the environment through analysis of their genome and transcriptome. The main goal of my dissertation work was to identify the changes occurring at the genome and transcriptome levels of eukaryotic cells when exposed to different commercial formulations of glyphosate (CFG). To achieve this, I exposed yeast isolated from different environments to CFG to identify the changes that occur at a cellular level. To further decipher the specifics of these changes, I put forth the following three specific aims for my dissertation work, which characterize the mechanisms of response and resistance to CFG.

\section{Specific Aim 1: Determine mechanisms and regulation of cellular transport of glyphosate using}

S. cerevisiae. The expression and maintenance of membrane proteins involved in the uptake, as well as exporting of glyphosate out of the cell, play a crucial role in the cell's ability to resist growth inhibition on exposure to CFG. Quantitative trait loci (QTL) analysis of strains with varying levels of sensitivity to Credit41 (commercial formulation of glyphosate used for the purpose of this study) in different environments (i.e., rich and minimal) provide insight into the loci contributing to the sensitivity of that strain. I measured the growth of yeast containing different alleles of the transporters in question to pinpoint which genes are involved in activities that aid in the tolerance of the cells to the chemical, i.e., glyphosate. I further studied the sensitive and resistant alleles and their corresponding levels of expression in different strains through reciprocal hemizygosity and qRT-PCR to decipher their correlation with resistance. 
Specific Aim 2: Identify genes contributing to Credit41-resistance. I used the genetic variation in S. cerevisiae to decipher the extent of contribution of the additives to cell toxicity. Using one commercial formulation as a representative of a few formulations with similar phenotypic responses, in this case Credit41 ( $\mathrm{Cr} 41)$, we analyzed the cells at the transcriptome level. Differentially expressed genes were studied to identify the pathways they were involved in, in turn understanding the response mechanisms in a sensitive and resistant strain. Whole-genome sequencing of cells adapted to $\mathrm{Cr} 41$ exposure was carried out to identify the changes at the genome level that resulted in this adaptation.

\section{Specific Aim 3: Determine the contribution of different cellular components regulating}

Credit41-resistance. A QTL analysis was performed between a vineyard strain, resistant to $\mathrm{Cr} 41$ and a Cr41 sensitive laboratory strain. The loci contributing to Credit41 resistance were identified and the specific genes within the loci responsible for the phenotype were identified. The candidate genes were then knocked out in both strains and the response to the environmental stressor were analyzed. Routes used by each strain were analyzed between the different experiments to understand the mechanisms involved in response and resistance to Credit41, and to what extent they vary between each strain. 


\section{References}

Amrhein, N., D. Johänning, J. Schab, and A. Schulz, 1983 Biochemical basis for glyphosatetolerance in a bacterium and a plant tissue culture. FEBS Lett. 157: 191-195.

Baerson, S. R., D. J. Rodriguez, N. a. Biest, M. Tran, J. You et al., 2002 Investigating the mechanism of glyphosate resistance in rigid ryegrass (Lolium ridigum). Weed Sci. 50: 721-730.

Healy-Fried, M. L., T. Funke, M. A. Priestman, H. Han, and E. Schönbrunn, 2007 Structural basis of glyphosate tolerance resulting from mutations of Pro101 in Escherichia coli 5enolpyruvylshikimate-3-phosphate synthase. J. Biol. Chem. 282: 32949-32955.

Mills, P. J., I. Kania-Korwel, J. Fagan, L. K. McEvoy, G. A. Laughlin et al., 2017 Excretion of the herbicide glyphosate in older adults between 1993 and 2016. JAMA - J. Am. Med. Assoc.

Powles, S. B., and C. Preston, 2006 Evolved Glyphosate Resistance in Plants: Biochemical and Genetic Basis of Resistance. Weed Technol. 20: 282-289.

Schütte, G., M. Eckerstorfer, V. Rastelli, W. Reichenbecher, S. Restrepo-Vassalli et al., 2017 Herbicide resistance and biodiversity: agronomic and environmental aspects of genetically modified herbicide-resistant plants. Environ. Sci. Eur. 29:. 


\section{CHAPTER 2: INTRODUCTION}

\section{Genetic Diversity within S. cerevisiae}

S. cerevisiae is one of the most extensively used eukaryotic model organisms for molecular and cellular biology studies, mainly due to its well-established cell architecture and highly conserved fundamental cellular mechanisms (Leland H. Hartwell et al. 2011). As a eukaryotic unicellular organism, it shares many metabolic pathways and complex cell structures with humans and other multicellular eukaryotes. Our extensive knowledge regarding the genetic make-up of yeast makes genetic manipulations easy to carry out. Another key feature that makes S. cerevisiae the ideal model organism for this study is the extent of genetic diversity within the species. Yeast toxicogenomic studies focus on analyzing the effects of different chemicals that we encounter on a regular basis, either directly or indirectly. We examine these effects in terms of changes in phenotype and variation in expression or alteration of the genotype. To decipher the different pathways involved, as well as to what extent these pathways are altered due to polymorphisms, it is convenient to carry out these studies in organisms with a wide range of variation. There are differences in each strain's ability to produce certain amino acids, and these auxotrophies can be utilized to our advantage to identify different pathways that are targeted. The alleviation of certain detrimental effects upon supplementation of these amino acids will be used as a confirmation of the pathway involved.

Different yeast strains have varying levels of sensitivity or resistance to different chemicals. A strain that is sensitive to glyphosate, may not necessarily be sensitive to other stressors; it's resistance profile is influenced by the environment it was exposed to (Landry et al. 2006; Smith and Kruglyak 2008). The variation between strains, in response to chemicals such as glyphosate-based herbicides, could be genetic or epigenetic, and are linked to previous exposure. Agricultural strains, like RM11 and AWRI1631, which have been exposed to glyphosate-based herbicides in the past, are more tolerant to 
glyphosate-based herbicides in comparison to laboratory (S288c) or clinical isolates (YJM789) (RongMullins et al. 2017a; Ravishankar et al. 2019). Yeast isolated in different decades make it possible to analyze the changes occurring in strains over a period of time in nature, answering important questions regarding adaptation mechanisms and a direct correlation with the changes occurring in the genome.

\section{Shikimate pathway}

Humans cannot synthesize nine of the 20 required amino acids; these are obtained through food intake. The aromatic amino acids are three of the essential amino acids in the human diet (Tzin and Galili 2010). These amino acids are obtained upon consumption of plants and fungi, which contain genes coding for the shikimate pathway, also known as aromatic amino acid biosynthesis pathway (Fig 1). The product of the shikimate pathway is chorismate, which serves as a precursor for these aromatic amino acids (Bentley and Haslam 1990). Phosphoenolpyruvate (PEP) and erythrose 4-phosphate are precursors that undergo a multi-step enzymatic reaction, resulting in chorismate. Chorismate then acts as a common precursor for the synthesis of the three aromatic amino acids, tryptophan (W), tyrosine (Y), and phenylalanine (F). This pathway is conserved across most plants, bacteria, and yeast, but not in other eukaryotes such as humans. The regulation of the shikimate pathway is highly complex, and it varies drastically from one organism to another. Though the basic principle of regulation is a feedback mechanism, the factors involved are different in each organism. In S. cerevisiae, Gcn4 is a transcription factor that binds to the promoter, regulating expression of the 3-deoxy-o-arabino-heptulosonate phosphate (DAHP) synthase gene (Viola et al. 2004). DAHP is the first intermediate product of the sevenstep shikimate pathway. The three aromatic amino acids also serve as building blocks for the production of many hormones and secondary metabolites (Herrmann and Weaver 1999), making it indispensable for growth of plants and most microorganisms. This aromatic amino acid biosynthesis pathway is targeted by glyphosate-based herbicides. 


\section{Glyphosate-based herbicides}

Glyphosate-based herbicides are used extensively around the world, due to their high levels of effectiveness as non-specific herbicides and very low animal toxicity. They are usually applied in concentrations ranging from 1.4-3.06 kg ha-1 application ${ }^{-1}$, depending on the crop being cultivated and soil type (Mamy et al. 2010). Glyphosate, a N-phosphonomethyl derivative of glycine, is the active ingredient in these herbicides. In the shikimate pathway, EPSPS (5-enolypyruvylshikimate-3-phosphate synthase) binds to phosphoenolpyruvate, one of its two substrates (Schönbrunn et al. 2001). Glyphosate binds to EPSPS to form a dead-end complex, which in turn inhibits chorismate production. Glyphosate and aminomethylphosphonic acid (AMPA), a primary metabolite of glyphosate, together have among the highest maximum residual limit, the maximum residual limit of up to $500 \mathrm{ppm}$ is permissible as per the Food and Agriculture Organization of the United Nations (Mesnage et al. 2015; Defarge et al. 2016). Some of the known effects of glyphosate exposure in plants are: rapid repression of photosynthesis, inhibition of $\mathrm{CO}_{2}$ assimilation, and a depletion of intermediates of the carbon reduction cycle (Faus et al. 2015). Other than the inhibition of aromatic amino acid biosynthesis, no other effects of glyphosate or the commercial formulations of glyphosate have been studied extensively in yeast.

There are multiple routes that can be taken to achieve glyphosate-resistance. These have been studied extensively in different organisms. Some of the resistance mechanisms are naturally occurring, as in the case of plants, as a survival mechanism. Others were studied by artificially modifying organisms, such as E. coli, in vitro. Some of the most commonly studied mechanisms to develop glyphosate resistance are target-site mutations and target-site duplication. This usually occurs through amino acid substitutions in the EPSPS enzyme, affecting glyphosate's ability to bind to it. Other mechanisms include the ability to degrade the herbicide through metabolism or increase the export of the herbicide from the cell (Sammons et al. 2007). As studies progressed, a number of non-target site 
mutations, and mechanisms other than metabolic regulation, were also found in response to this herbicide (Heap 2016). Initially, glyphosate-resistance was detected in Salmonella typhimurium with a P101S mutation in the EPSPS gene (Comai et al. 1983). Later, many sites apart from P101S were detected within the EPSPS gene in bacteria, and multiple simultaneous mutations within the EPSPS gene proved to be much more effective in conferring glyphosate-resistance (Padgette et al. 1991). With emerging off-target mutations, i.e., mutations in genes that lie outside the shikimate pathway, it is important to understand their effects in greater detail to continue the effective use of glyphosate-based herbicides.

\section{Commercial formulations and surfactants}

All commercial formulations of glyphosate-based herbicides are comprised of the active ingredient, glyphosate, along with additives and adjuvants. The main purpose of adding adjuvants to any herbicide or pesticide is to enhance the activity of the active ingredient, helping it pass the penetration barriers and come in contact with the target molecule. They also serve other purposes such as improving adsorption performance and rain fastness (Willoughby and Stokes 2015). There are different types of adjuvants, mainly surfactants, anti-foaming materials, buffering agents, etc., that are added depending on their purpose. Surfactants are commonly added to glyphosate-based herbicides to facilitate and accentuate emulsification, dispersion, and wetting, as well as carry out other surface modifying properties of liquids (Stanghellini and Miller 1997).

Depending on the particular commercial preparation, the surfactants added vary, and most of this information is proprietary. The laboratory studies usually use pure glyphosate that does not necessarily mimic the effects of the herbicide in the environment, or they fail to mention which formulation was used. This results in conflicting safety regulations due to contradicting results. Antifoaming agents, such as organo/modified polysiloxane, are added to some glyphosate-based herbicides. 
One common surfactant used in many of the glyphosate-based herbicides is polyoxyethylenamine (POEA). These adjuvants individually, as well as in combination with the other adjuvants present, are potentially teratogenic, having adverse effects on embryonic development (Mesnage et al. 2015). Studying the different formulations and at different concentrations is necessary to provide a more applicable insight into their effects.

\section{Yeast Growth media}

The ideal growth conditions for Saccharomyces cerevisiae are at $30^{\circ} \mathrm{C}$ in a high sugar

environment. The two most commonly used media to grow these cells are rich and minimal media. Rich media is commonly referred to as YPD (i.e., yeast-extract, peptone, dextrose). This media does not have any nutritional limitations because it contains everything most yeast strains require, in terms of nutrition, to grow. Yeast cells grow at a fast rate in this media, as the cells actively import amino acids from their environment.

Yeast minimal media, also known as YM, is comprised of nitrogen base and ammonium sulfate, along with sugar, minerals, and vitamins. To grow in this media, the cells need to synthesize their own amino acids by utilizing the nitrogen-base provided and their amino acid biosynthetic pathways. With the wide range of variation across different yeast strains, not all strains have the ability to produce the required essential amino acids (auxotrophies). In a case where a strain cannot produce a certain amino acid, to grow that strain in YM, the media has to be supplemented with that specific amino acid. Amino acid auxotrophies have been used as selectable markers for plasmid transformations and gene knockouts by replacement. When growing cells in this media, it is easy to have complete control of the environment that the cells are exposed to, as we know exactly what is being added to the media, which is not the case with YPD. 
In the case of glyphosate, which targets the chorismate pathway, the media can be supplemented with aromatic amino acids such as tryptophan, tyrosine, and phenylalanine (WYF) to bypass this effect. Also, using rich and minimal media to grow cells causes changes in the modes of cellular uptake of nutrients in the cell, in turn activating specific transport pathways.

\section{Cell wall and cell membrane}

The cell wall comes in contact with the environment containing chemicals like glyphosate and play an important role in the protection of the cell. The cell wall accounts for approximately $10-20 \%$ of a yeast cell's dry weight (Orlean 2012), hence, the cell puts in a considerable amount of metabolic energy into its biogenesis. The cell wall is highly elastic, and is an important contributor to the cell's permeability, shape and maintenance of integrity. The cell wall is mainly comprised of $\beta 1,3$ glucans and mannoproteins (Fig 2). Another important component of the cell wall is chitin, though it is a minor component, it is essential for the structural integrity of the cell wall (Kollár et al. 1997). These components are all linked together to form macromolecular complexes that are known as the flexible building blocks of the cell wall (Lipke and Ovalle 1998). The mannoproteins that lie in the cell wall are highly glycosylated polypeptides, which are comprised of a large number of serine and threonine residues (Orlean 2012).

The yeast cell wall is the outermost layer of the yeast cell. It is porous, and therefore, freely permeable to molecules smaller than 600Da; however, it is the cell membrane that provides stringency to the transport of molecules into and out of the cell. The cell membrane contains proteins that mediate the selective import and export of molecules for the cell. The cell membrane is comprised of a phospholipid bilayer containing the phospholipids, and proteins responsible for transport of molecules across the membrane (Carman and Henry 1989). It considers not only the size of the molecule, but also other characteristics, such as charge, shape, hydrophobicity, and other biochemical properties of the 
molecule. The yeast cell membrane contains ATP-driven transporters, that can broadly be classified into $A B C$ transporters and ion channels. The $A B C$ transporters are mostly highly conserved, and are responsible for the transport of drugs and amino acids across the membrane (Grenson et al. 1970; Balzi and Goffeau 1994). Being the outermost physical barrier, the cell wall is the first layer to come in contact with chemicals like herbicides in the environment.

\section{Genomic adaptation}

The ability of an organism to acclimate to perturbations encountered in its natural environment is crucial for its survival, making it necessary to develop mechanisms to cope with new situations quickly and effectively (Taymaz-Nikerel et al. 2016). Analyzing variation in the growth phenotype is first the approach to identifying the effects that different chemicals have on $S$. cerevisiae. A significant fraction of the phenotypic variation can be explained through variation at the genome and transcriptome levels. Characterization of the resulting changes sheds light on the metabolic pathways involved in the response and resistance mechanisms with respect to the stimuli. The changes at the cellular level, resulting in resistance, can be attained through changes in the genetic sequence, expression levels, or even epigenetic marks.

Genomic structural variations and point mutations are a crucial source of genetic diversity within an organism. These changes can occur in the form of Single Nucleotide Polymorphisms (SNPs), indels, or even through copy number variation (CNV). The occurrence of SNPs or indels, can give rise to a different allele of the gene, which can be crucial in altering the sensitivity of that cell to a certain environmental stimulus. Often times, these changes can have a neutral effect, depending if the mutation is synonymous or nonsynonymous. Even nonsynonymous mutations can, at times, have a neutral effect on the function of the protein. The effects of CNVs in yeast, on the other hand, are yet to be studied exhaustively, leaving gaps in knowledge in terms of its implications on the entire genome 
(Keel et al. 2016).Transposable elements and ncRNAs constitute common fragile sites in yeast (Casper et al. 2002). They are sites that are prone to breakages, which can result in duplication or CNV of genes that are flanked by these common fragile sites. 


\section{References}

Balzi, E., and A. Goffeau, 1994 Genetics and biochemistry of yeast multidrug resistance. BBA - Bioenerg. 1187: 152-162.

Bentley, R., and E. Haslam, 1990 The Shikimate Pathway - A Metabolic Tree with Many Branche. Crit. Rev. Biochem. Mol. Biol. 25: 307-384.

Carman, G. M., and S. a Henry, 1989 Phospholipid biosynthesis in yeast. Annu. Rev. Biochem. 58: 635669.

Casper, A. M., P. Nghiem, M. F. Arlt, and T. W. Glover, 2002 ATR regulates fragile site stability. Cell.

Comai, L., L. C. Sen, and D. M. Stalker, 1983 An Altered aroA Gene Product Confers Resistance to the Herbicide Glyphosate. Science 221: 370-371.

Defarge, N., E. Takács, V. L. Lozano, R. Mesnage, J. S. de Vendômois et al., 2016 Co-formulants in glyphosate-based herbicides disrupt aromatase activity in human cells below toxic levels. Int. J. Environ. Res. Public Health.

Faus, I., A. Zabalza, J. Santiago, S. G. Nebauer, M. Royuela et al., 2015 Protein kinase GCN2 mediates responses to glyphosate in Arabidopsis. BMC Plant Biol. 15: 14.

Grenson, M., C. Hou, and M. Crabeel, 1970 Multiplicity of the amino acid permeases in Saccharomyces cerevisiae. IV. Evidence for a general amino acid permease. J. Bacteriol. 103: 770-777.

Heap, I., 2016 International Survey of Herbicide Resistant Weeds. Online. http://www.weedscience.org/

Herrmann, K. M., and L. M. Weaver, 1999 THE SHIKIMATE PATHWAY. Annu. Rev. Plant Physiol. Plant Mol. Biol. 50: 473-503.

Keel, B. N., A. K. Lindholm-Perry, and W. M. Snelling, 2016 Evolutionary and functional features of copy number variation in the cattle genome. Front. Genet. 7:.

Kollár, R., B. B. Reinhold, E. Petráková, H. J. C. Yeh, G. Ashwell et al., 1997 Architecture of the Yeast Cell Wall. J. Biol. Chem. 272: 17762-17775.

Landry, C. R., J. Oh, D. L. Hartl, and D. Cavalieri, 2006 Genome-wide scan reveals that genetic variation for transcriptional plasticity in yeast is biased towards multi-copy and dispensable genes. Gene 366: 343-351. 
Leland H. Hartwell, F. H. C. R. C., T. I. for S. B. Leroy Hood, C. U. Michael L. Goldberg, F. H. C. R. C. Ann E. Reynolds, and P. U. Lee M. Silver, 2011 Saccharomyces cerevisiae: Genetic Portrait of a Yeast. Genet. From Genes to Genomes 1-20.

Lipke, P. N., and R. Ovalle, 1998 Cell wall architecture in yeast: New structure and new challenges. J. Bacteriol. 180: 3735-3740.

Mamy, L., B. Gabrielle, and E. Barriuso, 2010 Comparative environmental impacts of glyphosate and conventional herbicides when used with glyphosate-tolerant and non-tolerant crops. Environ. Pollut. 158: 3172-3178.

Mesnage, R., N. Defarge, J. Spiroux de Vendômois, and G. E. Séralini, 2015 Potential toxic effects of glyphosate and its commercial formulations below regulatory limits. Food Chem. Toxicol. 84: 133153.

Orlean, P., 2012 Architecture and biosynthesis of the Saccharomyces cerevisiae cell wall. Genetics 192: 775-818.

Padgette, S. R., D. B. Re, C. S. Gasser, D. A. Eichholtz, R. B. Frazier et al., 1991 Site-directed mutagenesis of a conserved region of the 5- enolpyruvylshikimate-3-phosphate synthase active site. J Biol Chem 266: 22364-9.

Ravishankar, A., A. Pupo, and J. E. G. Gallagher, 2019 Thickening of the cell wall increases the resistance of S. cerevisiae to commercial formulations of glyphosate. bioRxiv 760694.

Rong-Mullins, X., A. Ravishankar, K. A. McNeal, Z. R. Lonergan, A. C. Biega et al., 2017 Genetic variation in Dip5, an amino acid permease, and Pdr5, a multiple drug transporter, regulates glyphosate resistance in S. cerevisiae. PLoS One 12:.

Sammons, R. D., D. C. Heering, N. Dinicola, H. Glick, and G. A. Elmore, 2007 Sustainability and Stewardship of Glyphosate and Glyphosate-resistant Crops. Weed Technol. 21: 347-354.

Schönbrunn, E., S. Eschenburg, W. A. Shuttleworth, J. V Schloss, N. Amrhein et al., 2001 Interaction of the herbicide glyphosate with its target enzyme 5-enolpyruvylshikimate 3-phosphate synthase in atomic detail. Proc. Natl. Acad. Sci. U. S. A. 98: 1376-80.

Smith, E. N., and L. Kruglyak, 2008 Gene-environment interaction in yeast gene expression. PLoS Biol. 6: 810-824. 
Stanghellini, M. E., and R. M. Miller, 1997 BIOSURFACTANTS: Their Identity and Potential Efficacy in the Biological Control of Zoosporic Plant Pathogens. Plant Dis. 81: 4-12.

Tzin, V., and G. Galili, 2010 New Insights into the shikimate and aromatic amino acids biosynthesis pathways in plants. Mol. Plant 3: 956-972.

Viola, C. M., V. Saridakis, and D. Christendat, 2004 Crystal Structure of Chorismate Synthase from Aquifex aeolicus Reveals a Novel Beta Alpha Beta Sandwich Topology. Proteins Struct. Funct. Genet. 54: 166-169.

Willoughby, I. H., and V. J. Stokes, 2015 Mixture B New Formulation adjuvant increases the rainfastness and hence effectiveness of glyphosate for rhododendron control. Forestry 88: 172-179. 


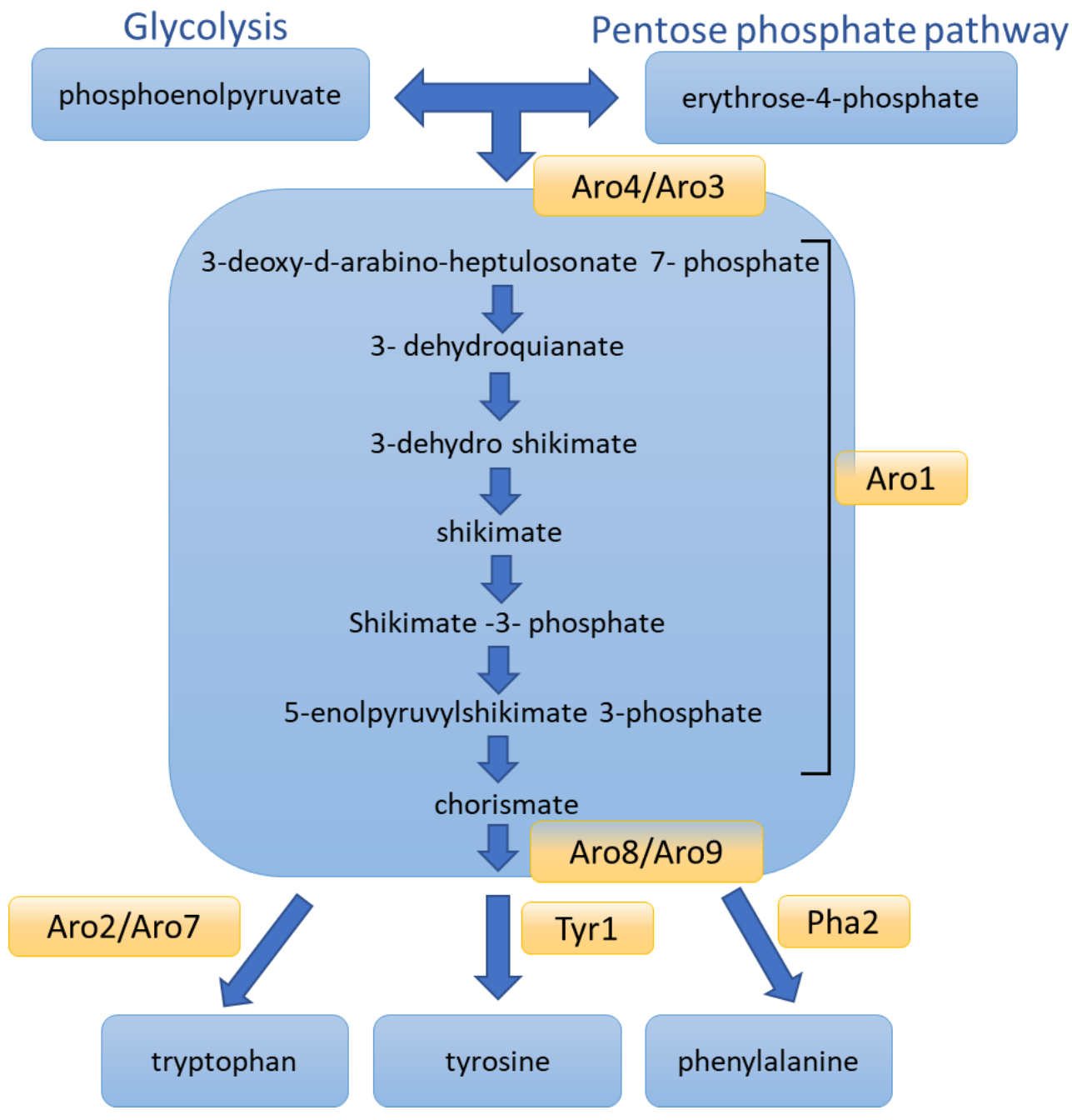

Figure 1: Aromatic amino acid biosynthetic pathway. Phosphoenolpyruvate and erythrose-4-phosphate are the primary substrates of the shikimate pathway. This pathway results in the production of chorismate, which gives rise to tryptophan $(W)$, tyrosine $(Y)$, phenylalanine $(F)$. The genes highlighted in the yellow boxes are the genes encoding the enzyme catalyzing that reaction in S. cerevisiae. 


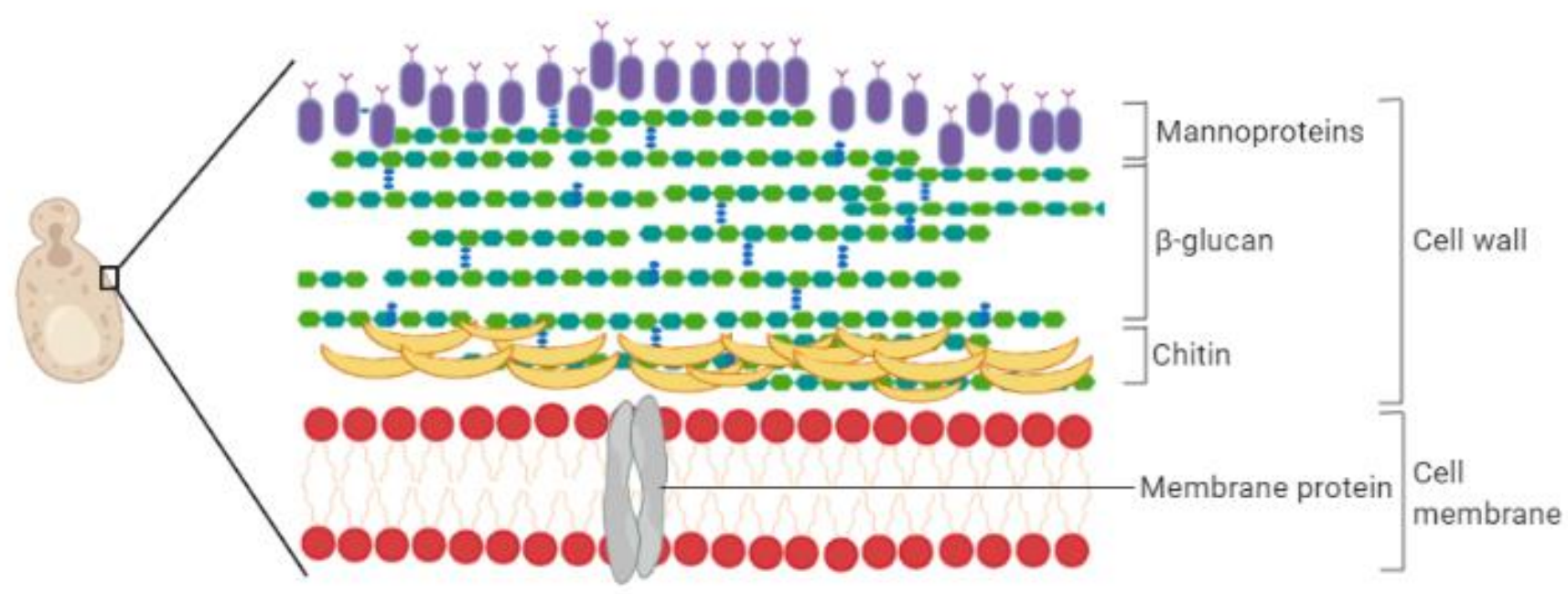

Figure 2: Saccharomyces cerevisiae cell wall and cell membrane structure. The S. cerevisiae cell wall is mainly comprised of $\beta$-glucans, mannoproteins and chitin. The cell membrane is a phosholipid bilayer that is interspersed with membrane proteins. 


\section{CHAPTER 3: GENETIC VARIATION IN DIP5, AN AMINO ACID PERMEASE, AND PDR5, A MULTIPLE DRUG TRANSPORTER, REGULATES GLYPHOSATE RESISTANCE IN S. CEREVISIAE}

A paper published in the journal PLOS ONE

Apoorva Ravishankarף, Xiaoqing Rong-Mullins $\rrbracket$, Kirsten A. McNeal ${ }^{\ddagger}$, Zachery R. Lonergan ${ }^{\ddagger}$, Audrey C. Biega ${ }^{\ddagger}$, J. Philip Creamer, and Jennifer E.G. Gallagher*

Ithese authors contributed equally

Xiaoqing Rong-Mullins

ROLES Investigation- QTL analysis

Apoorva Ravishankar

ROLES Investigation- DIP5 and PDR5, Validation, Writing - review \& editing

$\ddagger$ Kirsten A. McNeal, Zachery R. Lonergan, Audrey C. Biega, J. Philip Creamer

ROLES Investigation- $A R O 1$ investigation

Keywords: glyphosate, Aro1, shikimate pathway, Pdr5, Dip5

Running title: Genetic variation of yeast response to glyphosate

Abstract

S. cerevisiae from different environments are subject to a wide range of selective pressures, whether intentional or by happenstance. Chemicals classified by their application, such as herbicides, fungicides, and antibiotics, can affect non-target organisms. First marketed as RoundUp ${ }^{\mathrm{TM}}$, glyphosate is the most widely used herbicide. In plants, glyphosate inhibits EPSPS, of the shikimate pathway, which is present in many organisms but lacking in mammals. The shikimate pathway produces chorismate, which is the precursor to all the aromatic amino acids, para-aminobenzoic acid, and Coenzyme Q10. Crops engineered to be resistant to glyphosate contain a homolog of EPSPS that is not bound by glyphosate. Here, we show that S. cerevisiae has a wide-range of glyphosate resistance. Sequence comparison between the target proteins, i.e., the plant EPSPS and the yeast orthologous protein Aro1, predicted that yeast would be 
resistant to glyphosate. However, the growth variation seen in the subset of yeast tested was not due to polymorphisms within Aro1, instead, it was caused by genetic variation in an $A B C$ multiple drug transporter, Pdr5, and an amino acid permease, Dip5. Using genetic variation as a probe into glyphosate response, we uncovered mechanisms that contribute to the transportation of glyphosate in and out of the cell. Taking advantage of the natural genetic variation within yeast and measuring growth under different conditions that would change the use of the shikimate pathway, we uncovered a general transport mechanism of glyphosate into eukaryotic cells.

\section{Introduction}

RoundU $\mathrm{p}^{\mathrm{TM}}$ is a non-selective herbicide containing glyphosate and a variety of additives, such as detergents. As a broad-spectrum herbicide, glyphosate, the active ingredient in RoundUp ${ }^{\mathrm{TM}}$, inhibits production of chorismate, the precursor for tryptophan, tyrosine, phenylalanine, Coenzyme Q10, and para-Aminobenzoic acid (PABA), and its supplementation circumvents this growth inhibition in plants [1]. Glyphosate inhibits 5-enolpyruvylshikimate-3-phosphate synthase (EPSPS) in plants, an enzyme of the shikimate pathway (Fig 1A). Sensitive alleles of EPSPS are directly bound by glyphosate $[2,3]$. Changing amino acids in the glyphosate binding site of EPSPS or overexpression of EPSPS confers RoundUp ${ }^{\mathrm{TM}}$ resistance $[4,5]$. The functional ortholog of EPSPS in yeast is Aro1, and it contains additional enzymatic functions (S1A Fig) that are encoded by separate proteins in plants and bacteria [6]. 
Relatively low human acute toxicity, and the broad range of susceptible plants has encouraged widespread use of glyphosate, and its effects on non-target organisms are becoming pervasive [7]. Humans do not have EPSPS and cannot make aromatic compounds, and hence acquire these essential nutrients through their diet or microbiota $[8,9]$. The few reports of acute glyphosate toxicity in humans are likely from the detergents that are part of the commercial formulations [10]. Spraying glyphosate on crops and other weeds also exposes nearby organisms to glyphosate, including insects, bacteria, and fungi. Saccharomyces cerevisiae, a species with tremendous genetic diversity, occupies a wide range of niches, making it well suited for investigations of adaptation to new environmental stressors. There is more genetic diversity (SNPS/ Kb) between two different strains of yeast than among the entire human species [11-14]. There are 60,000 SNPs in the $12 \mathrm{Mb}$ yeast genome, [15] and 10 million SNPs in the 3,200 Mb human genome. [16]. Therefore, yeast is an ideal model organism to address acquisition of glyphosate resistance by examining variable alleles.

Two different mechanisms of glyphosate resistance have been uncovered in plants and bacteria. The first mechanism involves changing the ability of EPSPS to be bound by glyphosate, either by mutations that alter glyphosate binding efficacy (reviewed in [17]) or amplification of the EPSPS gene. The second mechanism involves increased levels of transport pathway elements such as $A B C$ transporters, which move glyphosate to the vacuole and, in turn, neutralize the effect of glyphosate [18]. Pdr5 is an ABC transporter that is orthologous to the human Mdr1, and is often amplified in chemotherapeutic resistant cancers [19]. 
To determine if there were other underlying mechanisms of tolerance, in this study, we characterized the yeast response to glyphosate. In different growth conditions, yeast exhibited a wide range of growth inhibition by glyphosate. The only known target of glyphosate is Aro1; however, polymorphisms in Aro1 were not responsible for the genetic variation of growth inhibition by glyphosate. Supplementing aromatic amino acids, which should bypass the shikimate pathway, improved growth in a dose-dependent manner, but there was still variation in growth. To address the media-dependent genetic variation in glyphosate tolerance, quantitative trait loci (QTL) analysis was carried out between two strains that demonstrated the greatest divergence in phenotypic response to glyphosate. In minimal media, the variation in glyphosate resistance was mapped to an amino acid permease, Dip5. While DIP5 deletion increased glyphosate resistance, expression of the resistant allele further improved growth of yeast on glyphosate, which suggested that the resistant allele has additional functions. Dip5 function was decreased by the addition of aspartic acid, resulting in relieved growth inhibition of all yeast tested in response to glyphosate. However, the magnitude of increased DIP5 mRNA levels in yeast that were treated with glyphosate did not correlate with changes in growth across strains. Together, this suggests that, regardless of the genetic variation in DIP5 among the various yeast strains, the Dip5 protein was similarly regulated by aspartic and glutamic acid. Dip5 can import glyphosate into the yeast cell, and the resistant allele has additional functions that the sensitive allele lacks in response to glyphosate. In addition, variation in glyphosate resistance in rich media was mapped to an $A B C$ pleiotropic drug transporter, Pdr5. Deletion of PDR5 resulted in the loss of glyphosate tolerance, while expression of the resistant allele 
conferred glyphosate resistance and expression of the sensitive allele did not. While it is likely that there are many proteins that regulate the response to and the transport of glyphosate, this study sought to identify the divergent genes between the two strains.

\section{Results}

There is a tremendous amount of phenotypic variation among yeast strains in response to different forms of stress. As rich media (YPD) contains all the required amino acids, it represses amino acid biosynthetic pathways and permits yeast to transport amino acids from the media into the cell. Six genetically diverse yeast strains isolated from different environments were grown on YPD supplemented with glyphosate (Fig 1B), RM11(a vineyard isolate), AWRI1631 (used for commercial wine making), S288c (a laboratory strain), YJM789 (a clinical isolate), UWOPS05.217.3 (isolated from bertam palm nectar), and YPS128 (isolated from the soil under an oak tree). These strains showed little change in growth on solid media in the presence of $0.1 \%$ volume/volume glyphosate $(78 \mathrm{mM})$ on YPD. The growth of YJM789, a clinical isolate, was reduced by the addition of $0.1 \%$ glyphosate in YPD (Fig 1B). At levels ten times higher, the growth of YJM789 was nearly completely inhibited, and the growth of S288c was slightly reduced. The commercial preparation of glyphosate contains additives, such as detergents, to increase the tissue penetration of glyphosate. To better reflect conditions that organisms would be exposed to, we chose to use the commercial preparation of glyphosate (RoundUp ${ }^{\mathrm{TM}}$ ) rather than pure glyphosate. In minimal media (YM), yeast were required to make all amino acids using ammonium sulfate as the sole nitrogen source. Under this growth condition, S288c, 
YJM789, and YPS128 were sensitive to $0.15 \%$ glyphosate, and all the strains tested, grew more slowly on $0.25 \%$ glyphosate treatment (Fig 1C). AWRI1631, RM11, and UWOPS05.217.3, which are agricultural isolates, demonstrated higher glyphosate tolerance in YM. Hence, among the agricultural isolates RM11 was found to be the most resistant to glyphosate exposure, followed by AWRI1631 and UWOPS05.217.3, whereas, YJM789 was the most sensitive, followed by YPS128 and S288c. When aromatic amino acids (tryptophan (W), tyrosine (Y), and phenylalanine (F)) were added back to YM (WYF), growth inhibition of S288c and YPS128 were alleviated (Fig 1C), and the growth of YJM789 showed improvement at lower glyphosate levels.

Numerous polymorphisms in the plant and bacterial homologs of Aro1 result in glyphosate resistance [17]. We predicted that yeast would be resistant to RoundUp ${ }^{\mathrm{TM}}$ by comparing mutations that have been mapped in AroA from $E$. coli and the yeast amino acid sequence of Aro1 [4, 20, 21] (S1B Fig). Yet, different strains of yeast showed differences in sensitivity to glyphosate that could be rescued with the supplementation of WYF. The polymorphisms in Aro1 across these six different yeast strains were outside the glyphosate binding domain (Fig 2A). To determine if polymorphisms in Aro1 contributed to the variation in glyphosate resistance, ARO1 was knocked out. The RM11 heterozygous aro1 $/$ /ARO1 strain was sporulated, and the tetrads were dissected onto YPD. A lethal mutation segregated in a 2:0 pattern that was linked to NatR in two independent knockouts, i.e., the aro1 $\Delta$ was inviable in RM11 (S1D Fig). NatR corresponds to a gene that provides resistance to nourseothricin. ARO1 was successfully knockout in S288c and YJM789 haploid yeast, and could grow if WYF was supplemented (Fig 2). 
To determine if $A R O 1$ was an essential gene in RM11, or if RM11 contained an allele that was synthetically lethal in combination with an aro1 deletion, RM11 (wild-type) was crossed with S288c aro1 (viable mutant), sporulated, and dissected onto YPD (S1E Fig). Fourteen tetrads were dissected, and 34 spores were viable. Twelve viable aro1 $\Delta$ segregants from the F1 hybrids ruled out the possibility that Aro1 ${ }^{\mathrm{RM} 11}$ gained an essential function, but instead aro1 $\Delta$ was synthetically lethal with an unknown allele present in the RM11 genome and not present in the S288c genome. The unknown allele was found to be unlinked to the size of the colony, the $M A T, H O$, and $A R O 1$ loci. In conclusion, the Aro1 deletion in most strains was found to be viable, with an exception of RM11.

To assess the impact of the genetic variation of Aro1 in response to glyphosate, four different alleles of $A R O 1$ were cloned under their endogenous promoter and terminator into plasmids and transformed into aro1 $\triangle$ yeast. ARO1 from AWRI1631 and RM11 represent alleles that are present in glyphosate resistant yeast, while ARO1 from S288c and YJM789 are alleles from less tolerant strains. While $A R O 1$ was essential in RM11, plasmids encoding alleles of $A R O 1$ were transformed into the heterozygous diploid, and the haploid knockouts with the plasmid were recovered after sporulation (Fig 2B). S288c aro1 $\Delta$ and YJM789 aro1 1 were viable on YPD and WYF but not on YM (Fig 2C and D). It can be concluded that all alleles of ARO1 could complement aro1 1 mutation in RM11, S288c, and $\mathrm{YJM} 789$ yeast because the aro1 1 yeast with plasmid expressed $A R O 1$ grew on YM (Fig 2) to similar levels as the wild-type parents carrying 
an empty plasmid. When grown on YM with glyphosate, yeast cells showed no difference in growth irrespective of the ARO1 allele expressed. However, S288c and YJM789, with ARO1 expressed from the plasmid, consistently grew slightly better than yeast expressing chromosomal ARO1. The mRNA levels of $A R 01$ from RM11, YJM789, and YJM789 aro1 $\triangle$ were quantified. In YJM789, ARO1 expressed from both the chromosomal location and from a plasmid was approximately the same (S1C Fig). There was a slight decrease in the levels of ARO1 mRNA found when YJM789 was exposed to glyphosate. In YJM789, expression of $A R O 1^{\mathrm{RM} 11}$ was no different from $A R O 1^{\mathrm{YJM} 798}$. Yet, there was an increase in ARO1 mRNA levels in RM11 compared to YJM789 in untreated growth conditions.

The primary target of glyphosate is inhibition of Aro1 in the chorismate pathway, and the genetic variation resulting in growth inhibition in response to glyphosate, was not due to genetic differences in Aro1. Therefore, the genetic variation in response to glyphosate could be due to genetic variation in other components of the chorismate pathway or in an unrelated pathway. To explore the differences in the chorismate pathway between S288c and YJM789, the aro1 knockouts were tested on various media. Chorismate is the precursor for aromatic amino acids, PABA, and coenzyme Q10 (CoQ10). para-aminobenzoic acid (PABA) is essential for the production of folic acid derivatives, and can be converted to Coenzyme Q10 precursor (Fig 1A). CoQ10 is involved in respiration, and is not essential for yeast viability. The commercial preparation of YM contains PABA and folate [22] and is noted here as YM+PABA. S288c aro1 $\Delta$ could not grow on WYF-PABA (YM without PABA, supplemented with WYF), while the wild-type parent could grow. In YJM789 aro1 , growth was only slightly reduced on WYF-PABA as 
compared to its wild-type parent; this difference in growth between S288c aro1 $1 \Delta$ and YJM789 aro1 $\Delta$ suggested the existence of an alternative pathway present in YJM789 that is absent in S288c. YJM789 was able to convert WYF to PABA and influence cell growth. RM11 required ARO1 for viability, and therefore, the knockout could not be tested (Fig 3A). Additionally, PABAfree and folate-free media was tested, where no change in growth was seen in comparison to PABA-free media, and thus this avenue was not further examined.

To determine if supplementation of yeast with downstream metabolites synthesized from chorismate could bypass the growth inhibition in the presence of glyphosate, yeast cells were grown on media supplemented with WYF in either the presence or absence of PABA. To assess whether PABA had a role in glyphosate growth inhibition, yeast cells were also tested on PABAfree media, supplemented with WYF. S288c aro1 $\Delta$ were more sensitive than the wild-type parent to glyphosate on WYF media (Fig 3B), while YJM789 aro1 $\Delta$ was more resistant to glyphosate than the wild-type parent. Only growth of RM11 on YM with glyphosate showed a slight decrease, while no change was detected when RM11 was grown in other conditions.

While genetic variation leads to differences in glyphosate resistance, the phenotypic response to glyphosate was not due to variation in ARO1. We chose to map the genes associated with glyphosate resistance in S288c and YJM789 because of the variation in their growth in YPD, WYF, and YM with glyphosate. We tested the growth of 125 recombinant haploid segregants from a hybrid of S288c and YJM789 [23]. For S288c in YPD with 1\% glyphosate, one locus of 
interest was identified on chromosome 15 with a LOD score of 35.5 (Fig 4A). PDR5, an ABC transporter that confers resistance to a wide-range of structurally diverse chemicals, [24, 25] was located within this region (Fig 4B). PDR5 has previously been implicated in plant response to glyphosate [18]. The role of each allele of PDR5 was tested in YJM789 and S288c diploid hybrids. PDR5 was knocked out from each parent and mated with the other parent. The resulting hemizygous strains are identical, except for the PDR5 allele that the hybrid inherited from one parent (Fig 4C). The $P D R 5^{\mathrm{YJM} 789} / \triangle$ hybrid was sensitive to glyphosate on YPD, while the $P D R 5^{\mathrm{S} 288 \mathrm{c}} / \Delta$ hybrid demonstrated the same level of tolerance as that of the wild-type hybrid. The sensitivity of the $P D R 5^{\mathrm{YJM} 789} / \Delta$ hybrid was similar to that of the homozygous knockout mutant. Pdr5 is a highly polymorphic protein, where the hyper-variability of its genetic sequence allows for greater specificity and tolerance to a wide array of chemicals per allele [26]. Between YJM789 and S288c, there is a 5\% difference in amino acid sequence. Among twelve available sequences that were analyzed, no obvious polymorphism was found in common among the yeast that were sensitive to glyphosate on YPD (S2 Fig).

Among the S288c and YJM789 segregants grown on WYF, an additional peak on chromosome 16 was found to be linked to glyphosate response (Fig 5A). The aforementioned peak on chromosome 15 associated with PDR5 in YPD was observed; however, the peak fell below the level of significance (LOD < 3.65) in YM with WYF. Additionally, the same peak on chromosome 16 was also identified when yeast were grown on YM without WYF supplementation (Fig 5B). Fourteen candidate genes were in the region under this peak (Fig $5 \mathrm{C}$ ), and were evaluated using the yeast knockout collection, where the likely candidate was DIP5 [27]. The knockout 
collection was constructed in an $\mathrm{S} 288$ c related strain, BY4741 that has multiple amino acid auxotrophies and several other differences from GSY147 (another S288c related strain) [28]. To circumvent the amino acid auxotrophies and achieve normal growth, BY4741 was supplemented with histidine, uracil, methionine, and leucine (HULM). BY4741 is highly sensitive to glyphosate and to accommodate for this, the level of glyphosate was reduced to $0.1 \%$. This reduction allowed for a more pronounced rescue of BY4741 dip5L.

Dip5 is a high affinity permease for aspartic and glutamic acid [29] and when these amino acids are in excess, the localization of the protein at the plasma membrane is reduced [30]. To assess if down-regulation of Dip5 at the plasma membrane could rescue glyphosate-induced growth inhibition, cells were grown in glyphosate media supplemented with aspartic acid. Aspartic acid (D) rescue was similar to that of WYF rescue and was specific. Whereas, the addition of other amino acids (HULM) did not rescue glyphosate sensitive yeast (Fig 5D). Aspartic acid is not a product of the shikimate pathway. Hence, the increased glyphosate tolerance of yeast when supplemented with aspartic acid suggests that glyphosate was imported into yeast via Dip5, where the increased tolerance is achieved through aspartic acid-mediated downregulation of Dip5. The role of each allele of DIP5 was tested in reciprocal hemizygous YJM789 and S288c diploid hybrids. DIP5 was knocked out from each parent, which was then mated with the other parent. The resulting hemizygous strains are identical except for the allele of DIP5 the hybrid inherited from one parent (Fig 5E). The wildtype hybrid, the homozygous mutant, and $D I P 5^{\mathrm{S} 288 \mathrm{c}} / \Delta$ hybrid were sensitive to glyphosate on minimal media, while the $D I P 5^{\mathrm{YJM} 789} / \triangle$ hybrid was resistant. The addition of WYF, supplements amino acids from the shikimate pathway. With 
$0.15 \%$ glyphosate, only the homozygous mutant diploid hybrid could be rescued by the addition of WYF. However, the addition of aspartic acid recused all strains.

As in the case of Pdr5, we could not identify a single polymorphism that was associated with all the resistant strains. However, as the presence of the DIP5 $5^{\mathrm{S288c}}$ allele decreased glyphosate resistance while presence of the DIP5 $5^{\mathrm{YJM} 789}$ conferred tolerance, the responsible polymorphism could be contributing to a hypomorphy-induced resistance-such as a mutation in the promoter sequence resulting in decreased expression of DIP5. This hypomorphy could be suspected to be responsible for the resistance of the YJM789 DIP5 allele, as DIP5 is expressed in levels greater than two-fold higher in S288c yeast as compared to YJM789 in YM [31]. In contrast, the expression of DIP5 increased in glyphosate treatment in YJM789 and to a lesser extent in AWRI1631 and RM11, but not S288c (Fig 6A). Growth inhibition of the other strains of yeast on exposure to glyphosate was rescued by addition of aspartic acid (Fig 6B). Additionally, the homozygous dip5 $\Delta$ mutant, did have improved growth with the addition of WYF compared to the DIP5 ${ }^{\mathrm{S} 288 \mathrm{c}}$ hemizygous diploid. To quantitate the ability of different amino acids to regulate the function of Dip5, quantitative growth assays were performed with two haploid S288c strains (BY4741 and GSY147). In the absence of glyphosate, deletion of DIP5 did not change the growth of either strain, except GSY147 dip5 $\Delta$ grew slightly slower than GSY147 in YPD (Fig 6C). Both dip5 strains grew better then wild-type controls when glyphosate was added (Fig 6C). The addition of aspartic acid downregulates Dip5 at the plasma membrane [30]. The improved growth of the dip5 $\Delta$ mutants on addition of aspartic acid suggests that import of 
glyphosate by Dip5 is a general mechanism, however there are other aspartic acid regulated modes of transport into the cell.

Because aro1 $\Delta$ mutants are sensitive to many drugs [32], we transformed alleles of PDR5 into both wild-type and aro1 $\triangle$ mutants to determine if expression of $P D R 5^{5288 c}$ could bypass sensitivity of aro1 1 in the presence of glyphosate. On YPD, both S288c and YJM789 aro1 mutants did not grow in the presence of glyphosate. Expression of each allele of PDR5 in YJM789 aro1 decreased growth in WYF media and completely abolished growth when supplemented with glyphosate (S4 Fig). Surprisingly, the YJM789 aro1 $\Delta$ mutant grew on WYF with glyphosate but not on YPD with glyphosate, where growth was abolished with the ectopic expression of any allele of PDR5.

\section{Discussion}

When grown in minimal media, the canonical target of glyphosate, the shikimate pathway, was affected and could be rescued by supplementation of downstream metabolites in most yeast. In this entire study, treatment with glyphosate implies treatment with a commercial formulation of glyphosate, Credit41. Utilizing an existing recombinant haploid yeast collection, two different loci linked to genetic differences in glyphosate response in S288c and YJM789 yeast were identified. Expression of $P D R 5^{\mathrm{S288c}}$ in YJM789 rescued sensitivity to glyphosate in rich media, while deletion in S288c conferred sensitivity. Pdr5 is the yeast ortholog of Mdr1, a multiple drug transporter that is highly polymorphic in yeast [15]. The second gene linked to 
glyphosate response was DIP5, which encoded an aspartic acid and glutamic acid permease [29]. The identification of these two genes furthers our understanding of the mechanisms by which glyphosate is transported in and out of the cell.

$\mathrm{S} 288 \mathrm{c}$ is a domesticated strain of $\mathrm{S}$. cerevisiae and has been the subject of extensive phenotypic, molecular and genetic analysis. Conducting studies on one genetic background limits the perspective. For example, aro1 mutants displayed differences in viability in RM11, S288c, and YJM789 strains and their growth on nutrient limiting media. Unlike S288c, ARO1 was essential for growth of RM11 on rich media but that was not from polymorphisms or a novel function in Aro1 ${ }^{\mathrm{RM} 11}$ but from a synthetic lethal interaction with an unknown allele in the RM11 genome. YJM789 aro1 mutants grew poorly on glyphosate and WYF alone, and were rescued by expressing an extra copy of PDR5 but only in WYF, when the function of Aro1 to produce chorismate was thought to be bypassed. RNA analysis of a slightly different glyphosate formulation found genes regulating membrane stress in response to glyphosate [33]. The sensitivity to the commercial preparation of glyphosate could only be in part a response due to other additives for two reasons. The yeast growth profiles were not the same in YPD and WYF and expression of Pdr5 alleles in WYF only showed a partial rescue. Previous studies did not directly identify the target of glyphosate in rich media, but protein coding polymorphisms in Pdr5 $5^{\text {S288c }}$ and Pdr5 ${ }^{A W R I 1631}$ would affect export of glyphosate. 
The best characterized target of glyphosate is 5-enolpyruvylshikimate-3-phosphate synthase in the shikimate pathway, and indeed all strains showed improved growth with WYF remediation. In addition, S288c and YJM789 aro1 1 cells grew slower on WYF with glyphosate than wild-type parental strains. This may be an indirect effect, as $\mathrm{S} 288 \mathrm{c}$ aro1 $\Delta$ yeast have been found to be sensitive to multiple drugs [32]. An alternative explanation is that there are other targets of glyphosate that become affected when the primary target of glyphosate is deleted.

Nevertheless, the increased toxicity of glyphosate when downstream metabolites of the shikimate pathway were provided, could be an indication of the presence of non-canonical glyphosate targets. Even sensitivity to glyphosate in rich media was unexpected. The shikimate pathway is down-regulated by the presence of aromatic amino acids, and the expression of Aro1 decreased in YPD compared to YM in S288c [34]. No change in Dip5 protein levels was detected in S288c by previous studies, while Pdr5 protein levels decreased in YM compared to YPD [34]. In rich media, nutrient transport pathways are unregulated and biosynthetic pathways are down regulated. While in minimal media, the opposite effect is observed in pathway regulation and expression. Also, there may be additional glyphosate-sensitive targets and future work will address their identification. Reports have found that glyphosate chelates calcium, manganese, iron, and magnesium [35] and glyphosate-sensitive soybean have lower levels of these minerals [36]. The shikimate pathway was originally identified years after the invention of glyphosate, by identifying increased levels of shikimate in glyphosate treated plants [2], similar methods may be applied to identify other affected pathways. 
Dip5 is located at the plasma membrane, and it transports aspartic acid and glutamic acid into the yeast cell. When there is excessive aspartic acid, Dip5 is targeted for endocytosis via arrestins and through ubiquitination it is targeted for degradation [30,37]. Deletion of DIP5 and expression of DIP5 $5^{\mathrm{YJM} 789}$ further increased glyphosate tolerance of the cell. In yeast cells that expressed only the DIP5 $5^{\mathrm{YJM} 789}$ allele, glyphosate resistance increased in YM, compared to yeast containing the DIP5 ${ }^{\mathrm{S288c}}$ allele which did not show the same. We proposed that Dip5 at the plasma membrane is at least one of the proteins involved in transporting glyphosate into the cell. This process is regulated by phosphorylations that promote ubiquitination [30], but there were no polymorphisms at any of these known residues (S3 Fig). Within the first 74 nucleotides there were three SNPs. From global transcriptomics [31] and mRNA expression it has been determined that the level of DIP5 mRNA is two-fold lower in YJM789. As S288c (GSY147) dip5D rescue was less pronounced than BY4741 dip5 $\Delta$ it can be concluded that Dip5 may be differently regulated between these strains. In addition, DIP5 ${ }^{\mathrm{S} 288 \mathrm{c}}$ may be regulated differently than DIP5 ${ }^{\mathrm{YJM} 789}$ because there was no rescue in DIP5 $5^{\mathrm{S288c}}$ with the addition of aspartic acid to levels of glyphosate tested here. During reciprocal hemizygosity we see yeast expressing DIP5 ${ }^{\mathrm{YJM} 789}$ was not the same as the knockout, which suggests that there is an additional function of DIP5 $5^{\mathrm{YJM} 789}$ compared to DIP5 ${ }^{\text {S288c }}$. The lower levels of Dip5 in YJM789 at the membrane which will internalize glyphosate may be downregulated faster than Dip5 ${ }^{5288 c}$. The addition of glyphosate increased the amount of DIP5 mRNAs in all the strains tested. The addition of aspartic acid rescued all strains including dip5 mutants suggesting that there are other transporters of glyphosate. 
In this study, we have uncovered one path of glyphosate import and one path of glyphosate export, and identified the differences within these transporters in the various strains. The polymorphisms in DIP5 and PDR5 propose a model for the entry and pumping out of glyphosate from the cell, respectively. We propose that polymorphisms, and differences in the Dip5 protein levels, change the amount of glyphosate transported into the cell. We hypothesize that Dip5 ${ }^{\text {YJM789 }}$ transports less glyphosate than Dip5 ${ }^{\text {S288C }}$ and both alleles are down-regulated by the addition of aspartic acid which could be through ubiquitination (Ub) and endocytosis. Once inside the yeast, glyphosate inhibits Aro1 and possibly other non-canonical targets. Either glyphosate or metabolized products are then transported out of the yeast by Pdr5 with the S288c allele being more active than the YJM789 allele. The allele present, in turn has a correlation with growth inhibition on exposure to glyphosate. Additional studies may reveal targets of glyphosate outside the shikimate pathway that could be classified as non-canonical targets. This study focused on differences of two yeast strains that varied in their glyphosate transport pathways. With the widespread use of glyphosate, encroachment of developments into pristine areas, and the efforts to control weeds and invasive species in state parks, glyphosate resistance is likely to continue its spread in the wild.

\section{Materials \& Methods}

\section{Yeast strains and plasmids}

Previously published strains and their derivatives are in S1 Table. ARO1, DIP5 and PDR5 were knocked out using homologous recombination with the dominant drug resistance NatR or KanR as previously described [38] and listed in S1 Table. In YJM789, DIP5 was replaced with 
dip5::KanR using BY4741 dip5 as the template and the following primers 5' AAA GTA CCA CAT ATC TAA CG 3' and 5' GTG ATA CCT GTA CAC TAT GGT TCC 3'. Cloning of ARO1 alleles was done by PCR amplified from genomic DNA using primers as follows 5'ARO1 5'ATG ACC ATG ATT ACG CCA AGC TTG CAT GCC TGC AGG TCG AGC CAA TCT CAC AGA TTT AAT ATA G3', 3'ARO1 5'TAT ATT GAT CAC CGA TAT ATG GAC TTC CAC ACC AAC TAG TAA TTC TTC AGT GAA TAA ACG GGC C3', 5'PDR5 5'GAT TAC GCC AAG CTT GCA TGC CTG CAG GTC GAC TCT AGA CTA ATC CAA TTC AGT TGT CTC3', and 3'PDR5 5'ATC ACC GAT ATA TGG ACT TCC ACA CCA ACT AGT TTC GGA CAG ATA ATG ATA TAA TAT ATC3'. All cloned genes were accompanied by their surrounding intergenic sequences up to the neighboring upstream and downstream genes. Genes were cloned via homologous recombination into the Xbal and Spel sites of pGS36 plasmid with hygromycin resistance [39]. Plasmids were kept under selection with hygromycin after LiAc chemical transformations [40]. ARO1 was knocked out with a PCR cassette containing NatR in YJM789K5a, GSY147, and RM11 MATa/MAT $\alpha$ and plated onto YPD containing $1 \mu \mathrm{g} / \mathrm{ml}$ nourseothricin. RM11 MAT $\alpha$ ho::KanR was crossed to GSY147 MATa, aro1:NatR and selected on YM with nourseothricin and G418. RM11 aro1 1 / ARO1 diploid carrying plasmids expressing ARO1 were sporulated and dissected onto YPD with hygromycin to select for the plasmid. There was genetic variation in tolerance of strains to hygromycin in YM. RM11 strains grown in YM required twice as much hygromycin $(250 \mu \mathrm{g} / \mathrm{ml})$ as required in YPD to maintain plasmids under hygromycin selection. Hemizygous hybrid yeast strains were constructed by transforming the wild-type parent with pGS36 and mating it with the mutant parent. Hemizygous yeast were selected with hygromycin and nourseothricin or G418 depending on the dominant selectable markers. The respective hemizygous genotypes and their markers are listed in S1 Table. The 
haploid recombinant segregant collection between YJM789 and S288c (S96) was previously generated [23].

\section{Media and chemicals}

All yeast strains were grown in nutrient rich media (YPD) or minimal media (YM) which includes $2 \%$ dextrose, $6.7 \mathrm{~g} / \mathrm{L}$ yeast nitrogen base and $20 \mathrm{~g} / \mathrm{L}$ agar in solid media. WYF plates contained 20 $\mu \mathrm{g} / \mathrm{ml}$ tryptophan, $30 \mu \mathrm{g} / \mathrm{ml}$ tyrosine and $50 \mu \mathrm{g} / \mathrm{ml}$ phenylalanine added to YM plates while D plates were supplemented with $100 \mu \mathrm{g} / \mathrm{ml}$ aspartic acid. Plates lacking para-Aminobenzoic acid (PABA) were made from yeast nitrogen base lacking PABA (Sunrise ${ }^{\circledR}$ ). Credit $^{\circledR} 41$ Extra $^{-1}$ contained $41 \%$ glyphosate and surfactants. The haploid recombinant segregant collection contained lys 2 and lys5 alleles segregating in the cross and therefore, lysine was added to media for these strains. YM and WYF media were also supplemented with histidine, uracil, leucine, and methionine for BY4741 strains. Yeast were grown overnight to saturation and then diluted to 0.1 OD units (approximately 1 X107 cells) and serially diluted 10-fold. Dilutions were then stamped on to plates. Growth was scored on solid media after 2-3 days of growth, relative to a control without glyphosate to account for how many of the spots grew. Quantitative growth assays were carried out in a TECAN automatic plate reader as previously described [41].

\section{RNA quantitation}


Total RNA was isolated by hot phenol extraction [42] and precipitated from cultures grown to mid-log phase and treated for 90 minutes with $0.25 \%$ glyphosate. RNA treated with DNAse I was then converted to cDNA using Invitrogen SuperScript ${ }^{\circledR}$ III First-Strand kit according to the manufacturer's directions. cDNA from biological duplicates was amplified in triplicate using specific primers in SSO FAST and normalized using a standard curve on a BioRad real-time PCR system. ARO1 primers used were upstream 5'ACCGACTGGTTAGGTATCCG3' and downstream 5'CCTAAACTGTGCAAGGCGTA 3'. 25S rRNA and genomic DNA were used to normalize samples with the following primers upstream 5'GACTACTTGCGTGCCTTGTTG3' and downstream 5'CCGTTCCCTTGGCTGTG3'. PDR5 primers used were 5'GTT GGC TGT TGG TGT TGC TA3' and downstream 5'AAC TAC AGG TGT CAG TGG CA3'.

DIP5 primers used were upstream 5'CTG CTG CTT TGG TCA TTC AA3' and downstream 5' TGG TTA GGA CCT CCA CCA AG3'.

\section{Mapping genetic linkage}

Quantitative trait loci (QTL) mapping for S96 x YJM789 haploid segregants in three different conditions was carried out as previously described [43]. The statistical threshold for each trait was calculated independently with 1000 permutations using R package qtl with EM method. Genes and coordinates under the peak of association for each condition was referenced using Saccharomyces Genome Database [44]. QTL scores are listed in S2-4 Table. 


\section{Acknowledgments}

We thank Ashok Bidwai for critical reading of the manuscript. Thanks to Luke Evans for suggesting the use of RoundUp ${ }^{\mathrm{TM}}$. Barbara Dunn and Gavin Sherlock provided the yeast used here and pGS36. Angela Lee generously shared the entire BY4741 yeast knockout collection. Zhenglong Gu and Xiaoxian Guo shared the YJM789 pdr5 knockout. West Virginia University PSCoR and West Virginia University Senate Grant provided initial funding. ACB was funded by WVU Summer Undergraduate Research Experience. ZRL was funded by NSF REU-Biological Responses to the Environment from Genes to the Ecosystem DBI 1156627. This work was funded by NSF-MCB 1614573. 


\section{References}

1. Haderlie LC, Widholm JM, Slife FW. (1972) Effect of glyphosate on carrot and tobacco cells. Plant Pathology 60(1): 40-3.

2. Amrhein N, Deus B, Gehrke P, Steinrücken HC. (1980) The site of the inhibition of the shikimate pathway by glyphosate. 66(5): 830-834.

3. Schönbrunn E, Eschenburg S, Shuttleworth WA, Schloss JV, Amrhein N, Evans JNS, et al. (2001) Interaction of the herbicide glyphosate with its target enzyme 5-enolpyruvylshikimate 3phosphate synthase in atomic detail. Proceedings of the National Academy of Sciences 98(4): 1376-1380. 10.1073/pnas.98.4.1376.

4. Healy-Fried ML, Funke T, Priestman MA, Han H, Schönbrunn E. (2007) Structural basis of glyphosate tolerance resulting from mutations of Pro101 in escherichia coli 5enolpyruvylshikimate-3-phosphate synthase. Journal of Biological Chemistry 282(45): 3294932955. 10.1074/jbc.M705624200.

5. Rogers SG, Brand LA, Holder SB, Sharps ES, Brackin MJ. (1983) Amplification of the aroA gene from escherichia coli results in tolerance to the herbicide glyphosate. Applied and Environmental Microbiology 46(1): 37-43.

6. Duncan K, Edwards RM, Coggins JR. (1987) The pentafunctional arom enzyme of saccharomyces cerevisiae is a mosaic of monofunctional domains. The Biochemical Journal 246(2): 375-86.

7. Tanney JB, Hutchison L. (2010) The effects of glyphosate on the in vitro linear growth of selected microfungi from a boreal forest soil. Canadian Journal of Microbiology 56(2): 138-44.

8. Reeds PJ. (2000) Dispensable and indispensable amino acids for humans. J Nutr 130(7): 1835S-40S.

9. Gill SR, Pop M, Deboy RT, Eckburg PB, Turnbaugh PJ, Samuel BS, et al. (2006) Metagenomic analysis of the human distal gut microbiome. Science 312(5778): 1355-1359. 312/5778/1355 [pii]. 
10. Bradberry SM, Proudfoot AT, Vale JA. (2004) Glyphosate poisoning. Toxicological Reviews 23(3): 159-67.

11. Liti G, Carter DM, Moses AM, Warringer J, Parts L, James SA, et al. (2009) Population genomics of domestic and wild yeasts. Nature 458(7236): 337-41.

12. Brem RB, Kruglyak L. (2005) The landscape of genetic complexity across 5,700 gene expression traits in yeast. Proceedings of the National Academy of Sciences of the United States of America 102(5): 1572-7.

13. Stranger BE, Forrest MS, Dunning M, Ingle CE, Beazley C, Thorne N, et al. (2007) Relative impact of nucleotide and copy number variation on gene expression phenotypes. Science 315(5813): 848-53.

14. Keinan A, Clark AG. (2012) Recent explosive human population growth has resulted in an excess of rare genetic variants. Science 336(6082): 740-3.

15. Wei W, McCusker JH, Hyman RW, Jones T, Ning Y, Cao Z, et al. (2007) Genome sequencing and comparative analysis of saccharomyces cerevisiae strain YJM789. Proceedings of the National Academy of Sciences of the United States of America 104(31): 12825-30.

16. International Human Genome Sequencing Consortium. (2004) Finishing the euchromatic sequence of the human genome. Nature 431(7011): 931-45.

17. Pollegioni L, Schonbrunn E, Siehl D. (2011) Molecular basis of glyphosate resistancedifferent approaches through protein engineering. The FEBS Journal 278(16): 2753-66.

18. Ge X, d'Avignon DA, Ackerman JJ, Sammons RD. (2010) Rapid vacuolar sequestration: The horseweed glyphosate resistance mechanism. Pest Manag Sci 66(4): 345-348.

19. Gottesman MM, Fojo T, Bates SE. (2002) Multidrug resistance in cancer: Role of ATPdependent transporters. Nature Reviews Cancer 2(1): 48-58.

20. Eschenburg S, Healy ML, Priestman MA, Lushington GH, Schönbrunn E. (2002) How the mutation glycine96 to alanine confers glyphosate insensitivity to 5-enolpyruvyl shikimate-3phosphate synthase from escherichia coli. Planta 216(1): 129-135. 
21. Funke T, Han H, Healy-Fried ML, Fischer M, Schonbrunn E. (2006) Molecular basis for the herbicide resistance of roundup ready crops. Proceedings of the National Academy of Sciences of the United States of America 103(35): 13010-5.

22. Marbois B, Xie LX, Choi S, Hirano K, Hyman K, et alClarke CF. (2010) Para-aminobenzoic acid is a precursor in coenzyme Q6 biosynthesis in saccharomyces cerevisiae. Journal of Biological Chemistry 285(36): 27827-27838. 10.1074/jbc.M110.151894.

23. Steinmetz LM, Sinha H, Richards DR, Spiegelman JI, Oefner PJ, McCusker JH, et al. (2002) Dissecting the architecture of a quantitative trait locus in yeast. Nature 416(6878): 326-30.

24. Leppert G, McDevitt R, Falco SC, Van Dyk TK, Ficke MB, Golin Jet al. (1990) Cloning by gene amplification of two loci conferring multiple drug resistance in saccharomyces. Genetics 125(1): 13-20.

25. Balzi E, Wang M, Leterme S, Van Dyck L, Goffeau A. (1994) PDR5, a novel yeast multidrug resistance conferring transporter controlled by the transcription regulator PDR1. Journal of Biological Chemistry 296: 2206-2214.

26. Guan W, Jiang H, Guo X, Mancera E, Xu L, Xu Let al. (2010) Antagonistic changes in sensitivity to antifungal drugs by mutations of an important $A B C$ transporter gene in a fungal pathogen. PloS One 5(6): e11309.

27. Giaever G, Chu AM, Ni L, Connelly C, Riles L, Véronneau S, et al. (2002) Functional profiling of the saccharomyces cerevisiae genome. Nature 418(6896): 387-91.

28. Winston F, Dollard C, Ricupero-Hovasse SL. (1995) Construction of a set of convenient saccharomyces cerevisiae strains that are isogenic to S288C. Yeast 11(1): 53-5.

29. Regenberg B, Holmberg S, Olsen LD, Kielland-Brandt MC. (1998) Dip5p mediates highaffinity and high-capacity transport of L-glutamate and L-aspartate in saccharomyces cerevisiae. Curr Genet 33(3): 171-177.

30. Hatakeyama R, Kamiya M, Takahara T, Maeda T. (2010) Endocytosis of the aspartic acid/glutamic acid transporter Dip5 is triggered by substrate-dependent recruitment of the 
Rsp5 ubiquitin ligase via the arrestin-like protein Aly2. Mol Cell Biol 30(24): 5598-5607. 10.1128/MCB.00464-10 [doi].

31. Zheng W, Zhao H, Mancera E, Steinmetz LM, Snyder M. (2010) Genetic analysis of variation in transcription factor binding in yeast. Nature 464(7292): 1187-91.

32. Hillenmeyer ME, Fung E, Wildenhain J, Pierce SE, Hoon S, Lee W, et al. (2008) The chemical genomic portrait of yeast: Uncovering a phenotype for all genes. Science 320(5874): 362-365. 10.1126/science.1150021 [doi].

33. Sirisattha S, Momose Y, Kitagawa E, Iwahashi H. (2004) Genomic profile of roundup treatment of yeast using DNA microarray analysis. Environ Sci 11(6): 313-23.

34. Newman JRS, Ghaemmaghami S, Ihmels J, Breslow DK, Noble M, DeRisi JL, et al. (2006) Single-cell proteomic analysis of S. cerevisiae reveals the architecture of biological noise. Nature 441(7095): 840-846.

35. Madsen HL, Christensen H, Gottlieb-Petersen C. (1978) Stability constants of copper (II), zinc, manganese (II), calcium, and magnesium complexes of $\mathrm{N}$-(phosphonomethyl) glycine (glyphosate). Acta Chem Scand 32: 79-83.

36. Cakmak I, Yazici A, Tutus Y, Ozturk L. (2009) Glyphosate reduced seed and leaf concentrations of calcium, manganese, magnesium, and iron in non-glyphosate resistant soybean. Eur J Agron 31(3): 114-119.

37. O'Donnell AF, Huang L, Thorner J, Cyert MS. (2013) A calcineurin-dependent switch controls the trafficking function of alpha-arrestin Aly1/Art6. J Biol Chem 288(33): 24063-24080. 10.1074/jbc.M113.478511 [doi].

38. Goldstein AL, McCusker JH. (1999) Three new dominant drug resistance cassettes for gene disruption in saccharomyces cerevisiae. Yeast 15(14): 1541-53.

39. Wenger JW, Schwartz K, Sherlock G. (2010) Bulk segregant analysis by high-throughput sequencing reveals a novel xylose utilization gene from saccharomyces cerevisiae. PLoS Genetics 6(5): e1000942. 
40. Gietz RD, Schiestl RH. (2007) Frozen competent yeast cells that can be transformed with high efficiency using the LiAc/SS carrier DNA/PEG method. Nature Protocols 2(1): 1-4.

41. Rong-Mullins X, Winans MJ, Lee JB, Lonergan ZR, Pilolli VA, Weatherly LM, et al. (2017) Proteomic and genetic analysis of S. cerevisiae response to soluble copper leads to improvement of antimicrobial function of cellulosic copper nanoparticles. Metallomics 9(9) 1304-1315.

42. Collart MA, Oliviero S. (2001) Preparation of yeast RNA. Current Protocols in Molecular Biology. John Wiley \& Sons, Inc. 10.1002/0471142727.mb1312s23.

43. Gallagher JE, Zheng W, Rong X, Miranda N, Lin Z, Dunn B, et al. (2014) Divergence in a master variator generates distinct phenotypes and transcriptional responses. Genes \& Development 28(4): 409-21.

44. Christie KR, Hong EL, Cherry JM. (2009) Functional annotations for the saccharomyces cerevisiae genome: The knowns and the known unknowns. Trends in Microbiology 17(7): 28694. 
A.
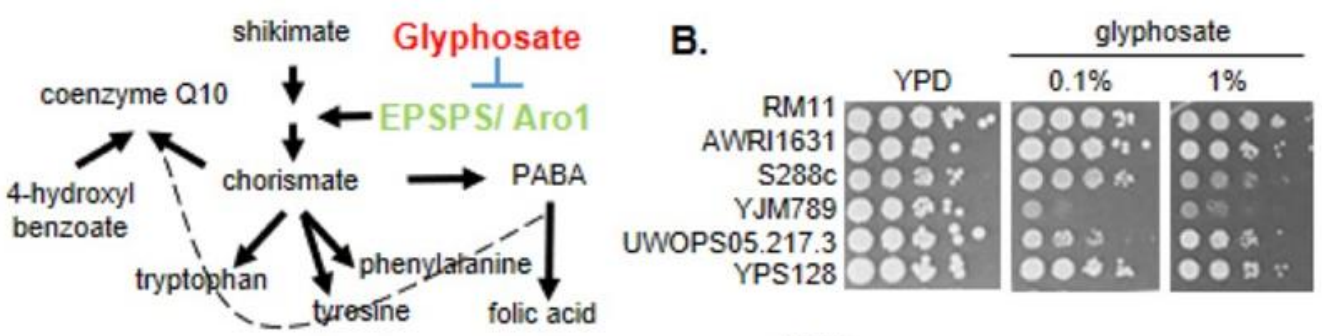

C.

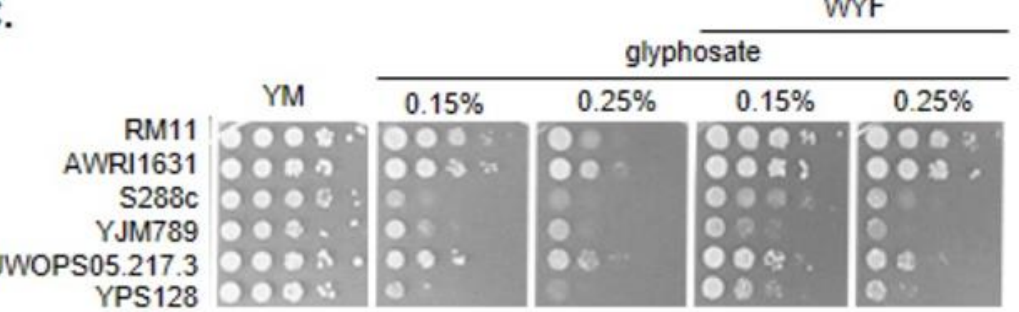

Fig 1 Genetic variation effects growth inhibition by glyphosate A. Shikimate pathway produces the precursor for phenylalanine, tyrosine, tryptophan, para-Aminobenzoic acid (PABA), folic acid and Coenzyme Q10. The canonical target of glyphosate is EPSPS in plants and Aro1 is the yeast homolog of EPSPS. PABA and 4-hydroxylbenzoate can be converted to Coenzyme Q10. B. Serial dilution of genetically diverse yeast on rich media (YPD) with dilutions of glyphosate as indicated (1\% vol/ vol is equivalent to $78 \mathrm{mM}$ ). C. Serial dilution of genetically diverse yeast on minimal media with glyphosate. Aromatic amino acids, tryptophan $(\mathrm{W})$, tyrosine $(\mathrm{Y})$ and phenylalanine $(\mathrm{F})$ were added to YM plates to make WYF.

(Survival assay performed by Kirsten McNeal) 
A.

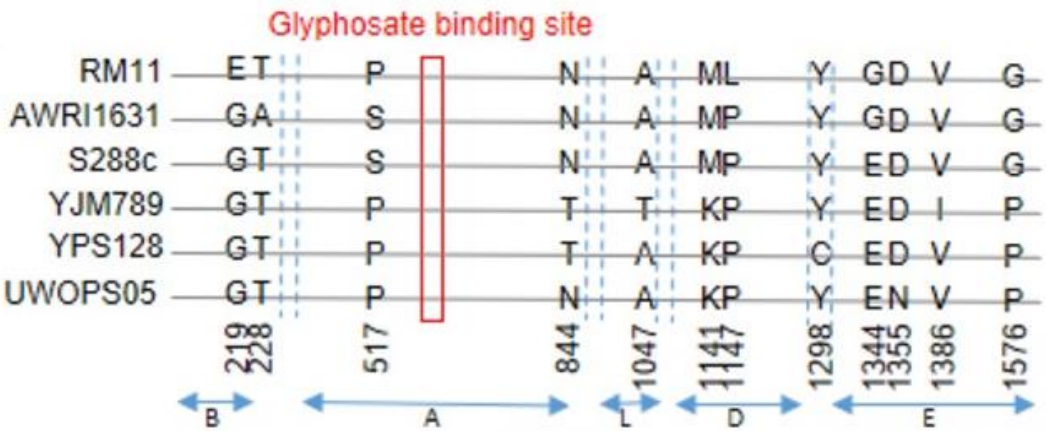

B.

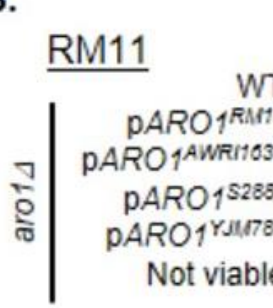

YPD

YPD $1 \%$ glyphosate $\quad$ YM

YM $0.15 \%$ YM $0.25 \%$

glyphosate glyphosate
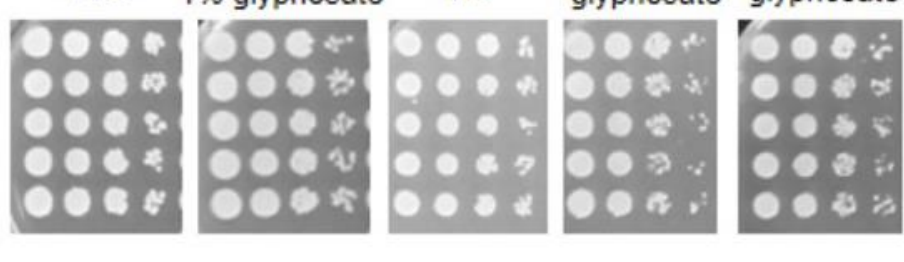

c.
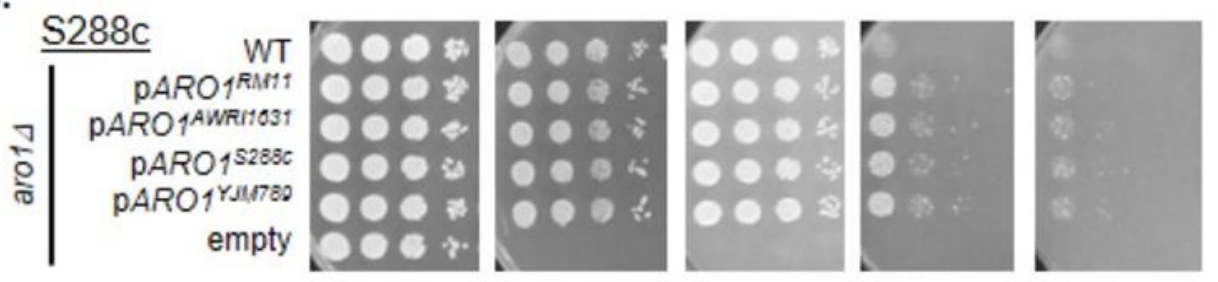

D.
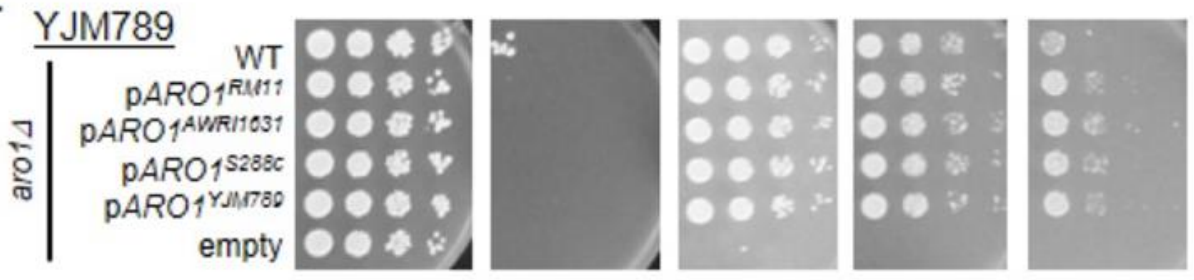

Fig 2 Contribution of the genetic variation within Aro1 to glyphosate resistance. A. Protein alignment of Aro1 from genetically diverse yeast strains. B. Serial dilutions of haploid aro1 $1 \Delta$ in RM11, C. S288c, and D. YJM789, expressing different alleles of plasmid encoded ARO1 were grown on YPD and YM with and without glyphosate. Rows labeled empty have pGS36 plasmid with no ARO1. Parental strains with the endogenous ARO1 expressed from the chromosome labeled WT carry an empty plasmid (pGS36).

(Survival assay performed by Zachary Lonergan) 


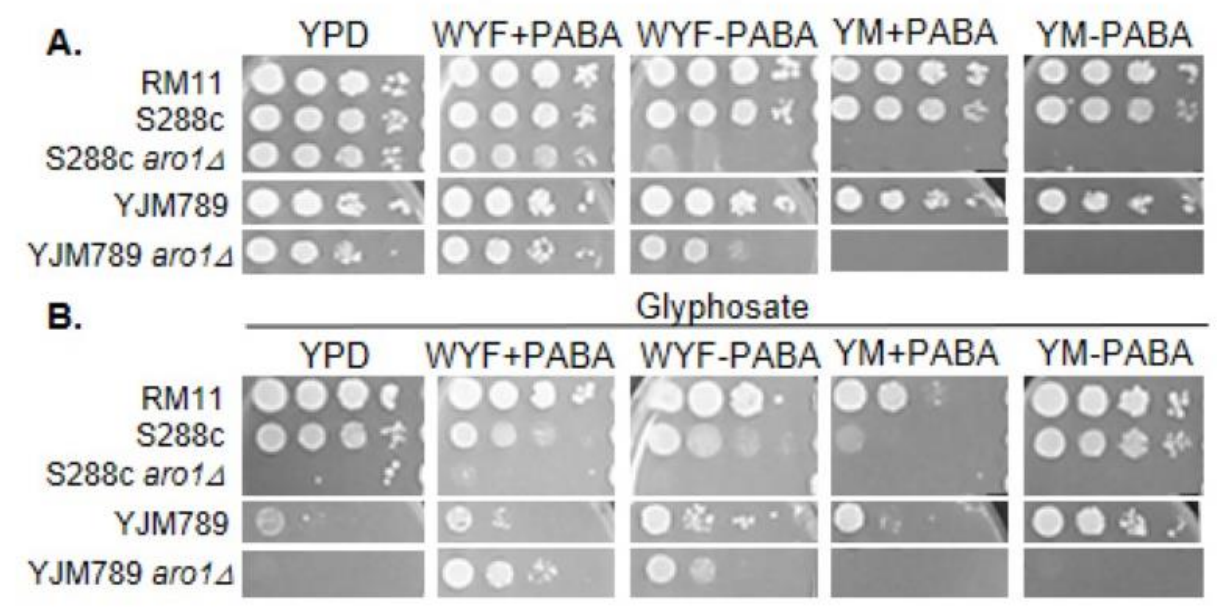

Fig 3 A. Different responses of aro1 $\Delta$ yeast on media supplemented with different aromatic metabolites. Wild-type RM11 was compared to S288c (GSY147) and YJM789 with and without $A R O 1$, three days in minimal media supplemented with aromatic amino acids (+WYF) or paraaminobenzoic acid (+PABA) or without these metabolites (-WYF or -PABA). B. Different responses of aro1 $1 \Delta$ yeast on glyphosate. Wild-type RM11 was compared to S288c (GSY147) and YJM789 with and without ARO1 on $0.15 \%$ glyphosate three days in minimal media supplemented with +WYF or +PABA or without these metabolites (-WYF or -PABA).

(Deletion mutants made and survival assay performed by Jennifer Gallagher) 


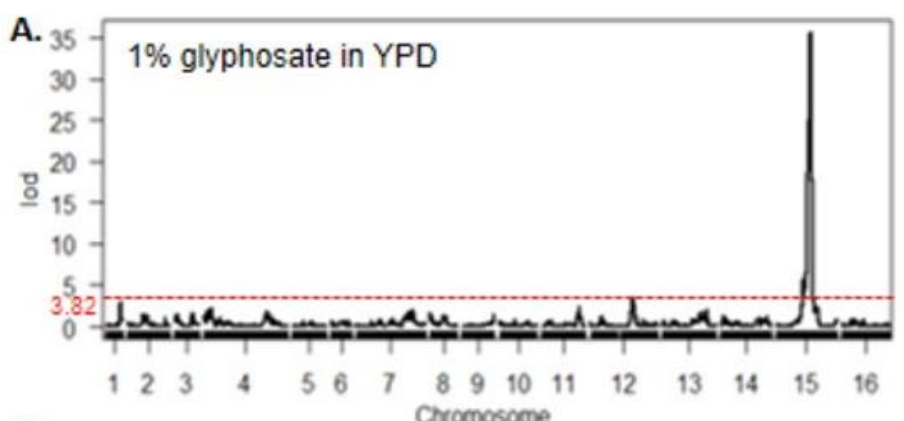

в.

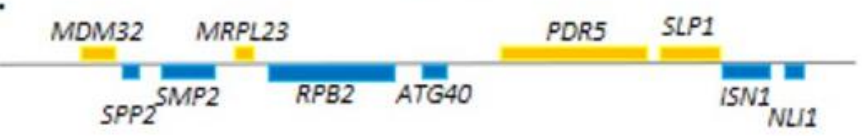

C.

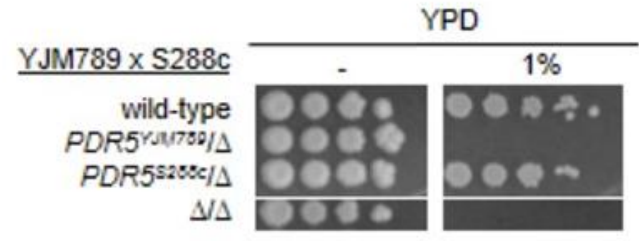

Fig 4 Genetic linkage analysis of glyphosate sensitivity in glyphosate on rich media A. Genetic linkage of sensitivity of YJM789 to $1 \%$ glyphosate in YPD. LOD score ( $y$-axis) was mapped across the yeast genome (x-axis) with chromosomes numbered left to right. The LOD significant levels (alpha = 0.05 ) was 3.82 and was marked by a red dashed line. $\mathbf{B}$. The genomic loci under the peak located on chromosome 15 contains 10 genes. Genes encoded on the top strand are in yellow and genes encoded on the bottom strand are blue. C. Serial dilution of S288c (GSY147) x YJM789 hybrids in which the entire PDR5 coding region in either parent was deleted $(\Delta)$, crossed, and the resulting hemizygotes were grown on rich media with and without $1 \%$ of glyphosate.

(Figure 4A QTL analysis was performed by Xiaoqing Rong-Mullins; Figure 4B and 4C, deletion mutants made, and follow-up analysis was performed by Apoorva Ravi Shankar) 
A.

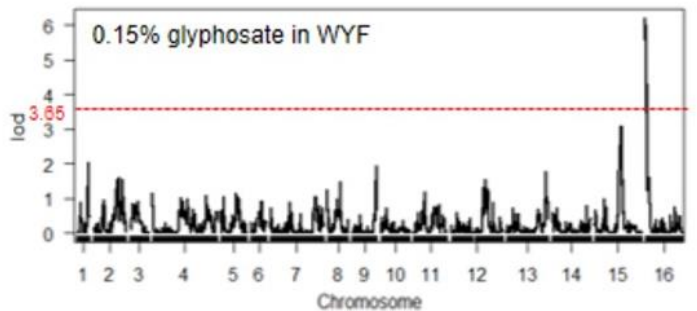

B.

C.
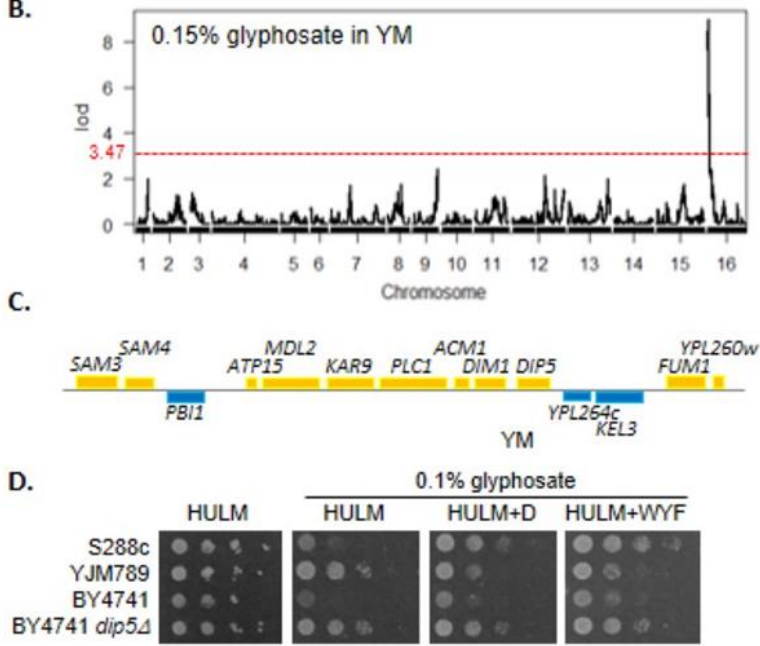

E.

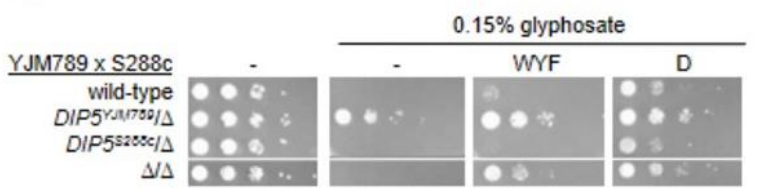

Fig 5 Genetic Linkage analysis of glyphosate sensitivity in glyphosate on minimal media with and without aromatic amino acids. A. Genetic linkage of sensitivity of YJM789 to $0.15 \%$ glyphosate in yeast minimal media supplemented with aromatic amino acids (WYF). LOD score (y-axis) was mapped across the yeast genome (x-axis). The LOD significant levels (alpha $=0.05$ ) was 3.65 and was marked by a red dashed line. B. Genetic linkage of sensitivity of YJM789 to $0.15 \%$ glyphosate in yeast minimal media (YM). LOD score ( $y$-axis) was mapped across the yeast genome ( $x$-axis). The LOD significant levels (alpha $=0.05$ ) was 3.47 and was marked by a red dashed line. $C$. The genomic loci under the peak located on chromosome 16 contains 14 genes. Genes encoded on the top strand are in yellow and genes encoded on the bottom strand are blue. D. Serial dilutions of S288c (GSY147), YJM789 and BY4741 with DIP5 knocked out grown on YM (HULM), WYF, aspartic acid (D) with glyphosate at the concentrations indicated. Histidine, uracil, leucine and methionine (HULM) were supplemented for growth of BY4741. E. Serial dilutions of S288c (GSY147) x YJM789 hybrids. The entire DIP5 coding region in either parent was deleted $(\Delta)$, crossed, and the resulting hemizygotes were grown on solid media YM, WYF, aspartic acid (D) with glyphosate at the indicated concentrations and supplemented.

(Figure 5A and 5B QTL analysis was performed by Xiaoqing Rong-Mullins; Figure 5C, 5D and 5E, deletion mutants made, and follow-up analysis was performed by Apoorva Ravi Shankar) 
A.

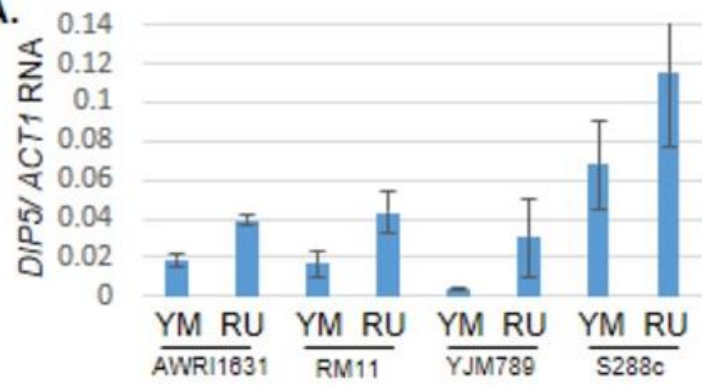

B.

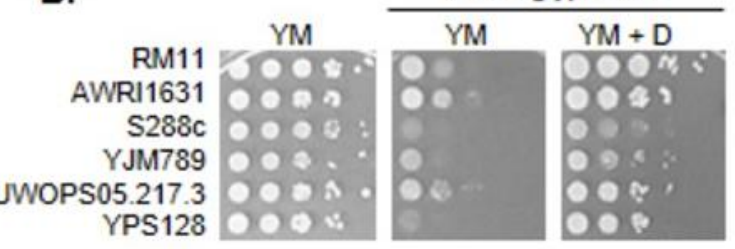

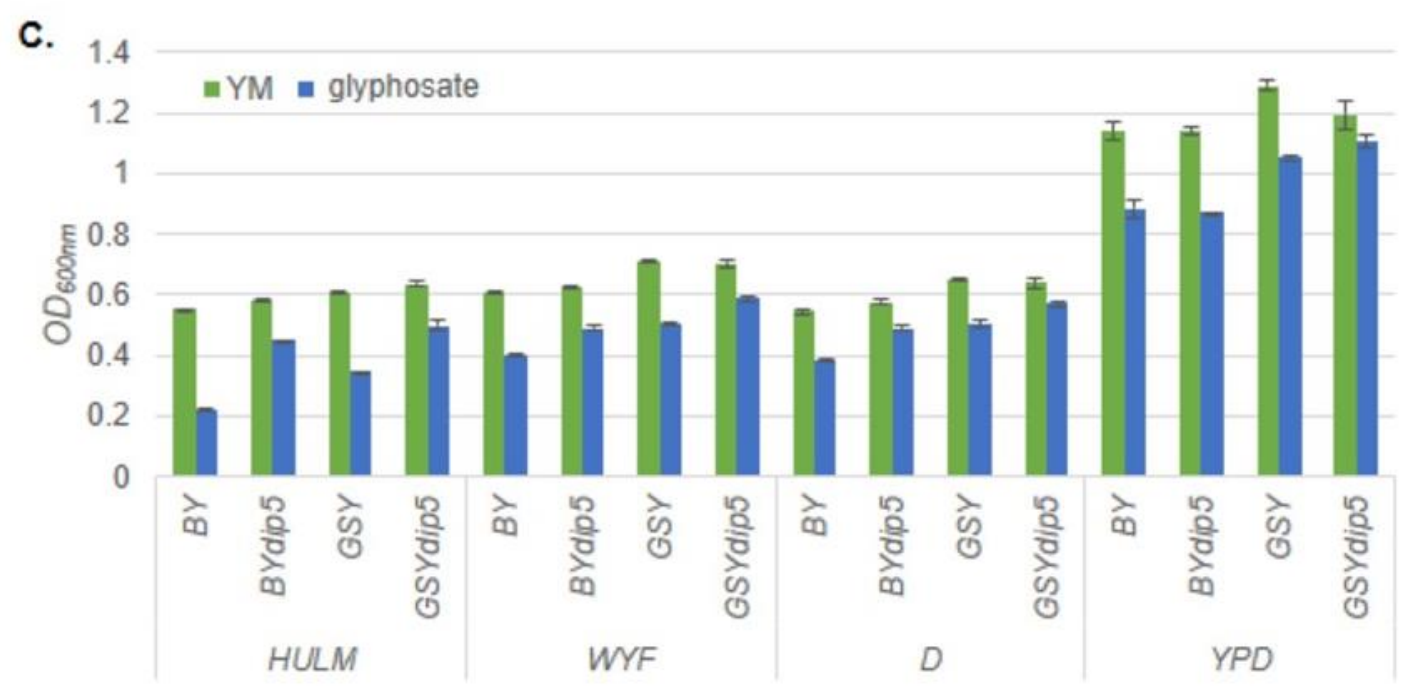

Fig 6 Regulation of DIP5 by glyphosate and aspartic acid. A. RNA expression levels of DIP5 mRNA in AWRI1631, RM11, YJM789 and S288c grown in YM with and without $0.25 \%$ glyphosate (RU). Q RTPCR of DIP5 mRNA levels are normalized to ACT1 mRNA. B. Serial dilution of genetically diverse yeast on minimal media with glyphosate supplemented with aspartic acid. C. Different responses of dip5 $\Delta$ yeast in liquid media supplemented with different aromatic metabolites and amino acids on exposure to glyphosate. BY4741 and GSY147 were grown in the presence of glyphosate $(0.1 \%$ in HULM, WYF and D and 1\% in YPD) and the optical density was measured in log phase (10 hr). (Figure 6 q-RT PCR and quantitative growth assay was performed by Apoorva Ravi Shankar) 


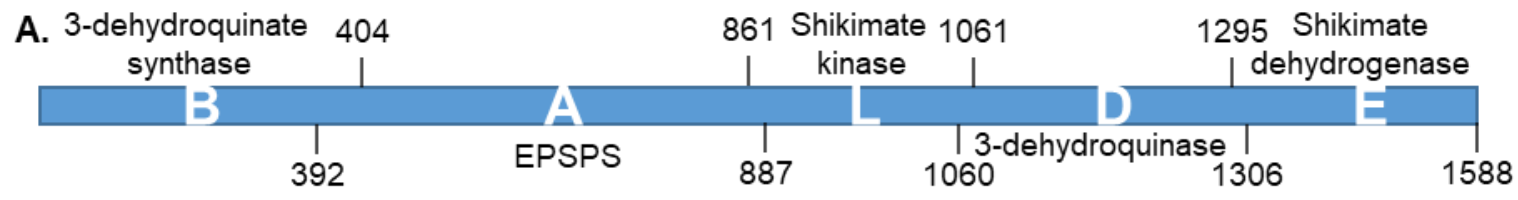

B.

\begin{tabular}{|c|c|}
\hline $\begin{array}{l}\text { Goose grass } \\
\text { Morning glory } \\
\text { Soybean } \\
\text { E. coli } \\
\text { Yeast } \\
\text { Agrobacteria }\end{array}$ & $\begin{array}{l}\text { NAGTAMRPLTA } \\
\text { NAGTAMRPLTA } \\
\text { NAGTAMRPLTA } \\
\text { NAGTAMRPLAA } \\
\text { TASRFLTSLAA } \\
\text { NAATGCRL-TM }\end{array}$ \\
\hline & ${ }^{*}{ }^{*}$ sं̄ \\
\hline
\end{tabular}

D. ARO1/ARO1
A
B
C
D
C.

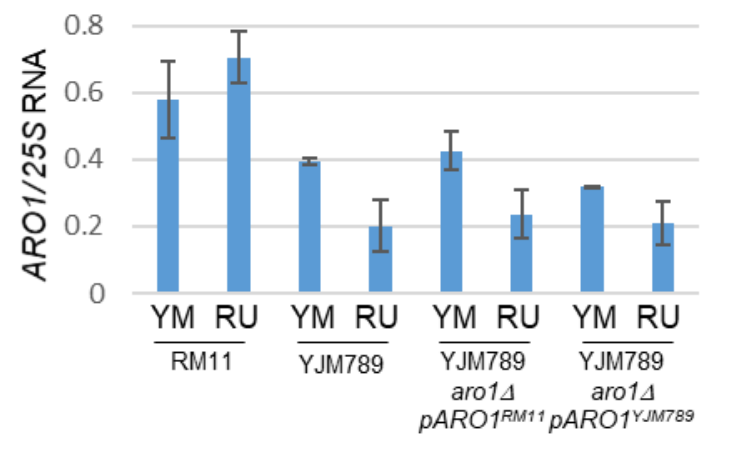

E.

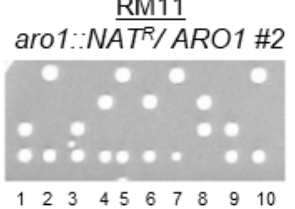

RM11 ARO1 x S288C aro1 $\because N A T^{R}$

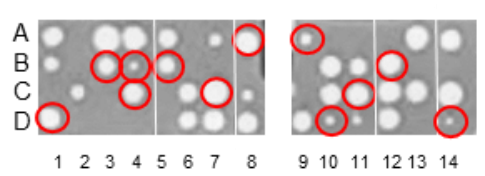

S1 Fig. Aro1 is a pentafunctional enzyme in the shikimate pathway. A. Schematic of enzyme functions in Aro1 with bacterial proteins AroB (3-dehydroquinate synthase amino acids 1-392), AroA (EPSPS amino acids 404-861), AroL (shikimate kinase amino acids 887-1060), AroD (3dehydroquinase amino acids 1061-1295), and AroE (shikimate dehydrogenase amino acids 13061599). B. Alignment of the EPSP glyphosate binding site across different species. In red are residues that when mutated confer resistance to glyphosate in E. coli. C. RNA expression levels of ARO1 mRNA from RM11, YJM789 and YJM789 aro1D carrying different alleles ARO1 grown in YM with and without $0.25 \%$ glyphosate. Q RT-PCR mRNA of ARO1 levels are normalized to $25 S$ rRNA. D. Tetrad dissections of RM11 heterozygous knockout of $A R O 1$ compared to wild-type RM11 diploid on YPD. Tetrads were numbered and haploid segregant germinating from a single spore are lettered. Plates were incubated at $30^{\circ} \mathrm{C}$ for two days. E. Tetrad dissections of RM11 wildtype and S288c aro1 $\triangle$ hybrids (F1) were incubated for five days before being photographed. Haploid segregants from F1 yeast with aro1 $1 \Delta$ were circled. 


\begin{tabular}{|c|c|c|c|c|}
\hline \multirow{10}{*}{ Pdr5 } & & $1 \%$ & $0.25 \%$ & $0.25 \%$ \\
\hline & & YPD & YM & WYF \\
\hline & $L 1528$ & 3.5 & 0.5 & 0.5 \\
\hline & RM11 & 4 & 1.5 & 0.5 \\
\hline & ]$_{B C 187}$ & 4 & 1.5 & 1 \\
\hline & $\Gamma K 11$ & 0 & 0 & 0.5 \\
\hline & L YJM339 & 3 & 3 & 1.5 \\
\hline & $\Gamma^{S 288 c}$ & 0 & 0.5 & 0.5 \\
\hline & $\|_{1 P S 128}$ & 3 & 0.5 & 3.5 \\
\hline & $I_{Y P S 163}$ & 3.5 & 2.5 & 3.25 \\
\hline & $D B V P G 6044$ & 2 & 0.5 & 0 \\
\hline & $T_{Y 55}$ & 4 & 3 & 4 \\
\hline & - UWOPSO52173 & 0.5 & 0 & 1.5 \\
\hline & YJM789 & 0.5 & 1.5 & 0.5 \\
\hline
\end{tabular}

S2 Fig. N-rooted phylogenetic tree of Pdr5 protein from genetically different yeast. Branch length was determined by UPGMA in ClustalW. Relative growth of yeast on glyphosate was normalized to growth with no treatment. 


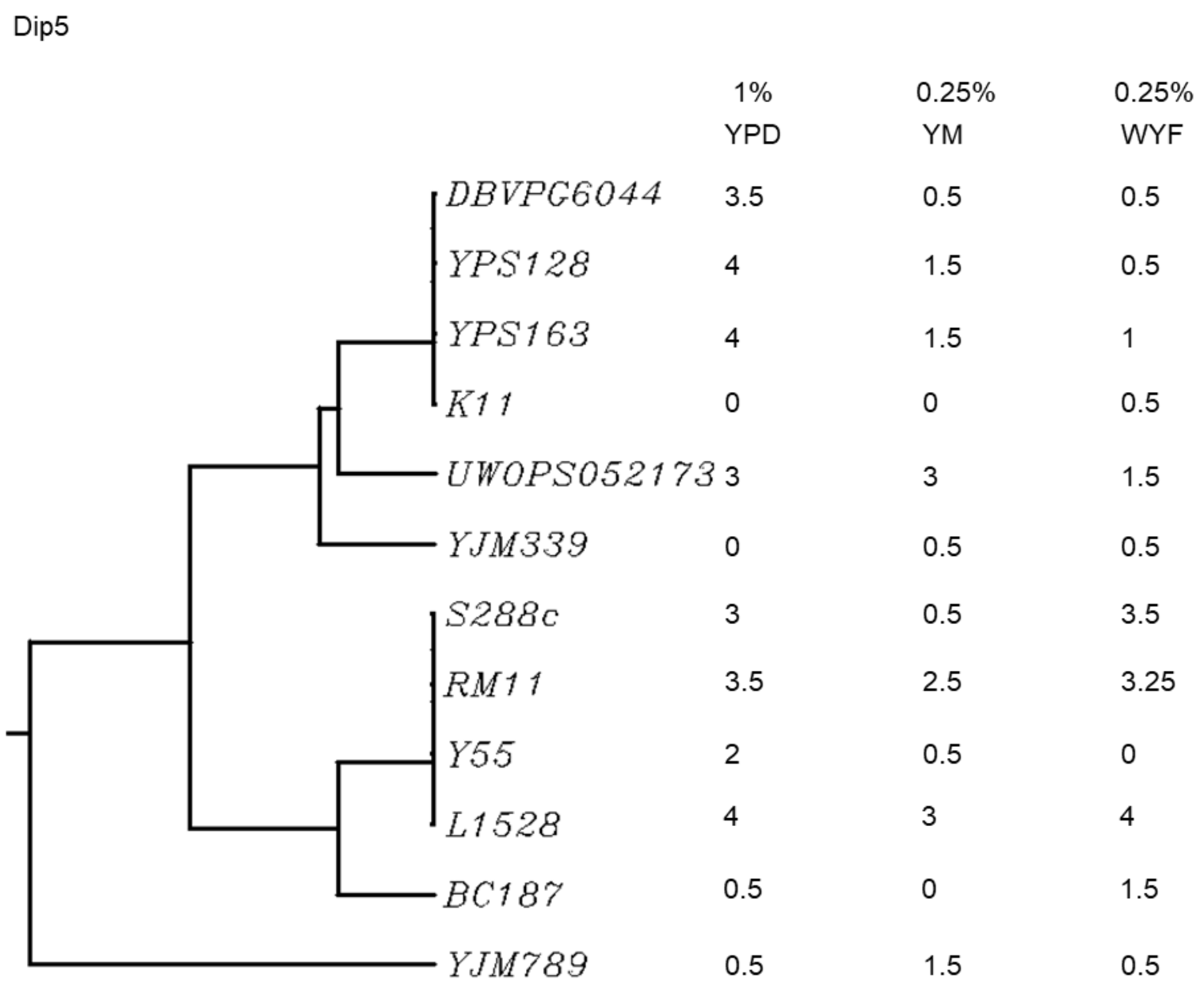

S3 Fig. N-rooted phylogenetic tree of Dip5 protein from genetically different yeast. Branch length was determined by UPGMA in ClustalW. Relative growth of yeast on glyphosate was normalized to growth with no treatment. 


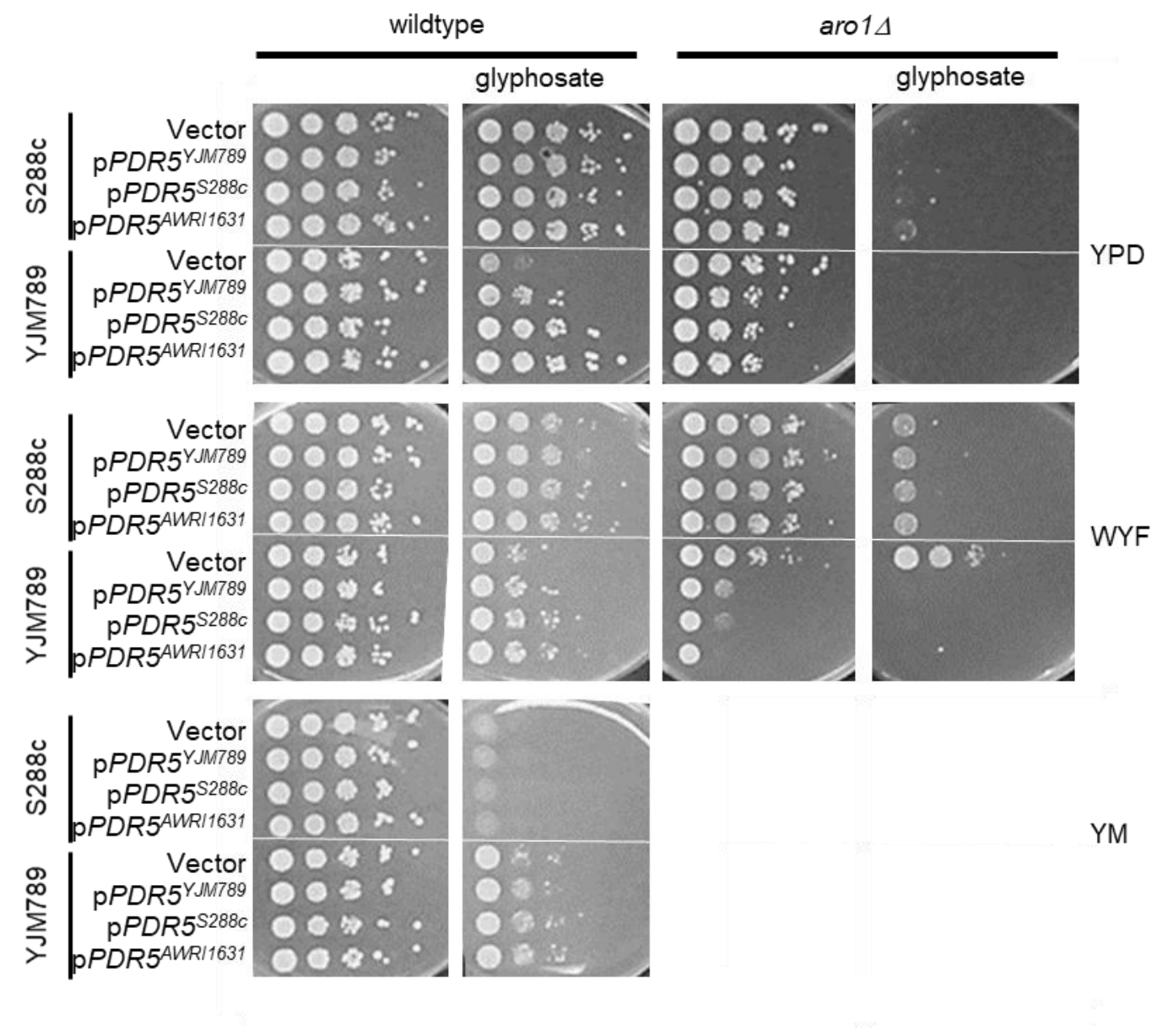

S4 Fig. Ectopic expression of PDR5 alleles in S288c and YJM789 with and without ARO1. ARO1 was knocked out in S288c and YJM789. PDR5 was cloned and expressed from its native promoter from a plasmid. Yeast were grown on YPD (rich media) with 1\% glyphosate, YM (minimal media) with $0.25 \%$ glyphosate and WYF (yeast minimal media supplemented with aromatic amino acids) with $0.25 \%$ glyphosate. (wild-type section of the figure made by Apoorva) 


\section{Supplemental Online resources}

Please use the links below to access the supplemental tables

S1 Table List of yeast used in this study.

https://doi.org/10.1371/journal.pone.0187522.s005

(XLSX)

S2 Table QTL mapping between S288c and YJM789 on 1\% glyphosate on YPD.

https://doi.org/10.1371/journal.pone.0187522.s006

(XLSX)

S3 Table QTL mapping between S288c and YJM789 on 0.15\% glyphosate on WYF. https://doi.org/10.1371/journal.pone.0187522.s007

(XLSX)

S4 Table QTL mapping between S288c and YJM789 on 0.15\% glyphosate on YM.

https://doi.org/10.1371/journal.pone.0187522.s008

$(\mathrm{XLSX})$ 


\title{
CHAPTER 4: THICKENING OF THE CELL WALL INCREASES RESISTANCE OF S. CEREVISIAE TO COMMERCIAL FORMULATIONS OF GLYPHOSATE
}

\author{
Apoorva Ravishankar, Amaury Pupo, and Jennifer E.G. Gallagher* \\ Paper submitted to G3: Genes, Genomes, Genetics
}

\begin{abstract}
The use of glyphosate-based herbicides is widespread, and despite its extensive use, its effects are yet to be deciphered completely. The additives in commercial formulations of glyphosate, though labeled as inert when used individually, have adverse effects when used in combination with other additives and the active ingredient. As a species, Saccharomyces cerevisiae has a wide range of resistance to glyphosate-based herbicides. To investigate the underlying genetic differences between sensitive and resistant strains, global changes in gene expression were measured when yeast were exposed to a commercial formulation of glyphosate (CFG). Changes in expression of genes involved in numerous pathways, such as DNA replication, MAPK signaling, meiosis, and cell wall synthesis were found. Because so many diverse pathways were affected, these strains were then subjected to in-labevolutions (ILE) to select mutations that confer increased resistance. Common fragile sites were found to play a role in adaptation mechanisms used by cells to attain resistance with long-term exposure to a CFG. The cell wall structure acts as a protective barrier in alleviating the stress caused by exposure to CFG; the thicker the cell wall, the more resistant the cell is against CFG. Hence, a detailed study of the changes occurring at the genome and transcriptome levels is essential to better understand the possible effects of CFG on the cell as a whole.
\end{abstract}




\section{Author Summary}

We are exposed to various chemicals in the environment on a daily basis. One such chemical that comes in direct contact with the food we consume are herbicides. This makes the study and thorough investigation of these chemicals crucial. Some of the most commonly used herbicides around the world are glyphosate-based. Their mode of action, affects a biosynthetic pathway that is absent in most higher eukaryotes and so it is deemed safe for consumption. However, there are many additives included in these herbicides to increase its effects. Thorough testing of these commercially available herbicides is essential to decipher all the potentially adverse effects that they could have on a cell. Saccharomyces cerevisiae has a wide range of genetic diversity, making it suitable to test different chemicals and identify any harmful effects. In this study, we exposed yeast cells to four glyphosatebased herbicides available in the market, to understand what effects they could have on a cell. We found that the additives in the herbicides have an effect on the cell wall and the mode of entry of glyphosate into the cell.

\section{Introduction}

Glyphosate based herbicides are among the most commonly used broad spectrum herbicides around the world (Duke and Powles 2008) because of its low toxicity to mammals, high efficacy, and affordability in comparison to other herbicides (Powles and Yu 2010; Duke 2018). Glyphosate acts by inhibiting the aromatic amino acid biosynthesis produced by the shikimate pathway. It targets the 5enolpyruvylshikimate-3-phosphate synthase (EPSPS) enzyme that plays a crucial role in the conversion of phosphoenolpyruvate (PEP) and 3-phospho-shikimate to phosphate and 5-enolpyruvylshikimate-3phosphate (Amrhein, et al. 1980; Powles \& Preston, 2006). Glyphosate binds to EPSPS in plants, in turn inhibiting the shikimate pathway and the production of tryptophan (W), tyrosine (Y), phenylalanine (F), parabenozic acid, and Coenzyme Q10 (Haderlie et al. 1977). 
Organisms undergo different modes of adaptation to attain resistance to an environmental stressor. In the case of glyphosate-based herbicides, the routes to attaining resistance are classified into two categories: 1. Target site associated resistance, and 2. Non-target site resistance (Yuan, et al. 2007). Target site associated resistance is comprised of resistance mechanisms involving changes in the EPSPS gene and the shikimate pathway. Typically, either the glyphosate binding site in EPSPS is mutated or EPSPS is over expressed. Non-target site resistance is attained due to changes outside the shikimate pathway. One of the most commonly studied forms of non-target site resistance is vacuole related changes (Hereward et al. 2018; Moretti et al. 2017). Recent studies showed that genes involved in glyphosate transport are one of the non-target resistance mechanisms (Rong-Mullins, Ravishankar et al. 2017). Some yeast strains encode a CFG resistant pleiotropic drug response protein, Pdr5 (Rong-Mullins, Ravishankar et al. 2017). The Pdr5 protein is involved in transport of glyphosate and CFG additives out of the cell. Another non-target resistance mechanism comes from the protein Dip5, a glutamic and aspartic acid permease; its deletion leads to glyphosate tolerance (Rong-Mullins, Ravishankar et al. 2017).

There are hundreds of commercial formulations of glyphosate (CFG) available in the market. The most challenging aspect of studying the effects of these CFG is that the label lists the concentration of glyphosate present in the mixture but does not provide details regarding the adjuvants added or the concentrations they are added in. It is crucial to monitor and study these CFG as a whole, along with the adjuvants, as the additives often alter the effectiveness of the principal component and other factors such as its extent of biodegradability ( Mesnage et al. 2013). Some of the identified additives are polyoxyethylamines (POE), quaternary ammonium compounds, and some heavy metals (Defarge et al. 2018; Nicolas Defarge et al., 2016; Nørskov et al. 2019). As the contributions of these additives to the toxicity of the herbicide have recently been uncovered, they are now being studied in more detail (Jacques et al. 2019). However, there is still a large gap in knowledge in terms of the changes that these additives inflict on the genome and transcriptome of organisms. A diverse group of stressors triggers the 
environmental stress response (ESR), which is seen in the change of global, yet similar pattern of gene expression. The changes in transcript levels of genes observed in the state of ESR are involved in various cellular processes, such as energy generation and storage, defense against reactive oxygen species, protein folding, DNA repair, etc., and the protection of these pathways are vital to the proper functioning of the cell (Gasch et al. 2000).

The yeast cell wall is the first physical barrier of the cell to encounter any environmental stressor. The yeast cell wall is $15-30 \%$ of the dry weight of the cell (Orlean 1997). The cell wall is mainly comprised of an inner layer made of polysaccharides and an outer scaffold made of mannoproteins (Klis, et al. 2002; Orlean, 2012; Stewart \& Stewart, 2018). The mannoproteins, $\beta-1,3$ glucans and $\beta-1,6$ glucans form the major components of the cell wall, with chitin forming non-covalent bonds with some glucans as a minor component. The cell wall is highly dynamic in nature and it can adapt to various physiological and morphological conditions (Aguilar-Uscanga and François 2003). The cell wall functions to stabilize internal osmotic pressure, protect the cell against mechanical injury and chemical stress, maintain the cell shape, and provide a scaffold for glycoproteins (Stewart and Stewart 2018). The outer protein layer of the cell wall contains at least 70 proteins, which may vary depending on the environment and the type of stressors the cells are exposed to (De Groot et al. 2005; Zlotnik et al. 1984). Proteins containing a large amount of $\mathrm{N}$ - and $\mathrm{O}$-linked mannoses are called mannoproteins, many of which are found on the outermost layer of the cell wall (Zlotnik et al. 1984). Some of these structural cell wall proteins are rich in serine and threonine, and undergo a post translational addition of a glycosyl phosphotidylinositol (GPI) anchor (Nuoffer et al. 1991). Sed1 is one of many GPI-anchored mannoproteins in the cell wall bound to a glucan. Stationary phase cells show an increase in Sed1 levels (Shimoi et al. 1998). Cells in stationary phase tend to be much more resistant to various environmental stressors (WernerWashburne et al. 1993) and induction of these proteins is usually observed on exposure to stress (Shimoi et al. 1998). 
S. cerevisiae has a wide range of genetic variation within the species. Some of this variation pertains to the origin of these strains, in terms of their prior exposure to different stressors. In this study, we used the genetic variation in yeast to assess the effects of CFG on the transcriptome of different strains of yeast. We also analyzed various modes of attaining resistance to CFG that lie outside the shikimate pathway. CFG caused a pleiotropic response in exposed cells. They affected numerous pathways involved in various vital processes for the cell's survival such as, meiosis, DNA replication, cell wall proteins, MAPK and HOG signaling pathways, etc. The high osmotic response (HOG pathway) aids in survival of the cell in case of cell wall stress (García et al. 2009). Cell wall structure plays an important role in resistance to CFG. Some cell wall proteins such as Sed1, when expressed in high numbers, contributed to the thickness of the cell wall and aided in tolerance to CFG. Another interesting aspect was the contribution of Ty elements to adaptation of tolerance mechanisms against CFG. Numerous genes that lie between Ty elements underwent duplication during the process of developing adaptation mechanisms on CFG exposure. Many of these changes could have a cumulative response in the cells developing resistance against CFG.

\section{Results}

\section{Variation in growth in the presence of glyphosate-based commercial formulations}

Four genetically diverse haploid strains with well annotated genomes (S1 Table (Song et al. 2015)) were chosen to test for differences in growth when exposed to different commercial formulations of glyphosate (CFG). RM11 is a vineyard strain (Mortimer et al. 1994; Török et al. 1996) and AWRI1631 is descended from a commonly used strain for commercial wine manufacturing (Borneman et al. 2008). YJM789 is derived from a clinical isolate, isolated from an immunocompromised AIDS patient (McCusker et al. 1994; Tawfik et al. 1989). The last strain included was a commonly used laboratory strain with an S288c background (GSY147) (Engel et al., 2014a; R K Mortimer \& Johnston, 1986; Wenger 
et al. 2010). These strains were exposed to commercial formulations of glyphosate-based herbicides on solid media, with pure glyphosate as a control. We exploited this variation to decipher the effects of the different commercially available formulations of glyphosate-based herbicides. There are a large number of formulations available, many of which contain a mixture of multiple active ingredients, such as pelargonic acid, diquat, imazapic, and other chemicals ("Corn and soybean herbicide chart" 2013). The ones selected for this study were chosen because they were labeled as containing glyphosate as the sole active ingredient in the herbicide, hence the variation observed is the clear effect of the putatively inert ingredients such as adjuvants.

When grown with CFG on rich media (YPD), AWRI1631 and RM11 continued to grow in the presence of 1\% glyphosate in all the formulations (Fig 1A), whereas, YJM789 and S288c displayed a growth defect, and S288c was extremely sensitive to only Weed Pro (WP) as compared to the other three formulations. On minimal media (YM), where cells synthesize their own amino acids from the nitrogen-base provided, ammonium sulfate in this case, there was greater variation between the formulations evn on use of lower concentrations of glyphosate, especially in the case of WP where all the strains were sensitive; however, the pattern was generally consistent among the other formulations, with S288c showing maximum sensitivity and AWRI1631 showing resistance to a larger extent (Fig 1B). As glyphosate targets the aromatic amino acid pathway, supplementing the minimal media with tryptophan, tyrosine, and phenylalanine (WYF) should bypass the inhibition of the shikimate pathway and restore growth. However, rescue with WYF and the CFG was variable, and growth improved to a great extent though the cells did not fully recover (Fig 1C). AWRI1631 and RM11 growth inhibition was less compared to YJM789 and S288c. S288c was the most effected by all the formulations. All the strains were sensitive to WP, and supplementing WYF did not show considerable rescue in this formulation. This indicates that there are other factors influencing the cell growth other than the active ingredient, and these vary from one formulation to the other. Dip5 is an amino acid permease that allows entry of 
glyphosate into the cell (Rong-Mullins et al. 2017a). It is downregulated in the presence of excess aspartic acid (Hatakeyama et al. 2010) and adding back aspartic acid did rescue the cells significantly, but not entirely in all the formulations, especially in case of S288c (Fig 1D). The addition of excess aspartic acid to cells treated with pure glyphosate rescued the growth inhibition. In case of CFG, the alleviation of growth inhibition was not much with addition of aspartic acid. YJM789 was an exception to this, as there was a significant rescue on treatment with Credit $41(\mathrm{Cr} 41)$ when supplemented with aspartic acid. The growth inhibition resulting from exposure to WP was alleviated to a greater extent on addition of aspartic acid in comparison to WYF, but not completely. In comparison, when yeast were grown on pure glyphosate (PG) the variation in growth was similar to that of $\mathrm{Cr} 41$, Compare and Save (CAS), and Super Concentrated (SC) but to a lesser extent, which supports the hypothesis that the additives do contribute to growth inhibition. YJM789 was the exception as it was the most sensitive to the pure glyphosate in all conditions. To consider a formulation that is representative of a phenotype similar to most commercial formulations, $\mathrm{Cr} 41$ was used for all further experiments.

\section{Polygenic trait with a strain, and condition-based pattern in response to $\mathrm{Cr} 41$}

There are tens of thousands of SNPs between these four strains. To explore how genomes change to permit adaptation to high levels of $\mathrm{Cr} 41$, the yeast were serially passaged in media containing Cr41 by performing In-Lab-Evolutions (ILEs). Three biological replicates were passaged in YM, YM supplemented with WYF and YPD media, with $0.25 \%$ glyphosate from the $\mathrm{Cr} 41$ in minimal media and $1 \%$ in rich media respectively (Fig $2 \mathrm{~A}$ ). The strains were passed through six passages by transferring $1 \%$ culture to fresh media. Once the resistant populations were identified, single colonies were isolated. These strains were then released from the selective pressure for two passages and then the $\mathrm{Cr} 41$ resistance was confirmed (Fig 2B-E). This step was performed in order to ensure that the resistance wasn't entirely due to epigenetic mechanisms that cannot be detected through whole-genome sequencing. The resistant cells sequenced for each strain along with the condition are listed in S2 Table. 
The ILEs selected a large number of SNPs as well as duplicated regions. The synonymous mutations were filtered out and only the genes containing non-synonymous mutations were taken into consideration. A total of 148 genes (S3 Table) accumulated at least one non-synonymous SNP within the coding region among all the sequenced strains treated with $\mathrm{Cr} 41$. The genes that accumulated more than one SNP and/or indel in any of the samples from different strains were prioritized and focused on for this study. Dip5 in part transports glyphosate into the cell as shown in our previous study (Rong-Mullins et al. 2017a), and contained SNPs in three of the sequenced samples (Fig S1).

Principal Component Analysis (PCA plots) were plotted for each sample taking all the SNPs into consideration in order to identify the major sources of variation. The main reason behind doing this was to ensure that the main sources of variation corresponds to the biological conditions and was not a mere effect of experimental bias. An R-package, pcadapt (Luu et al. 2017) visualized the patterns that naturally exist between the strains. Hence, resistant strains clustered together and so did the sensitive ones when the data from all the conditions was pooled together (Fig $3 \mathrm{~A}$ ). The underlying background genetic differences between each strain dominated the genome comparisons. Further analysis of the strain specific PCA plots showed that the clustering of the controls vs. the Cr41 treated samples in S288c was based on PC2 (Fig 3B), and PC1 did the same in the case of RM11 (Fig 3C).

Sections of the genome containing as few as two to as many as a hundred genes increased in copy number (CN) as shown by copy number variation analysis (CNV). All the strains in this study were haploid and having a $\mathrm{CN}$ of less than one can only occur at nonessential genes. In this analysis any genes that were partially duplicated were excluded under the assumption that the change in copy number is associated with synteny or the other genes present in their immediate surroundings (S4 Table). Any gene that underwent a CNV in a treated cell but also had an increase in $\mathrm{CN}$ in any control sample, was filtered out. This made it apparent that there is an underlying connection between the strain and the condition (i.e. YM, YM with WYF or YPD) it was evolved under. As in the case of YJM789, CNVs occurred 
only in cells that were evolved in minimal media with WYF. S288c had the most genes (81 genes) that underwent CNV while RM11 did not have any, which was one of the most CFG resistant strain. As per the Venn-diagram, some of the S288c genes that underwent CNV were found in all the conditions, but many of which were found in cells evolved in minimal media supplemented with WYF. Though these genes underwent duplication in a specific condition (i.e., WYF), they did not belong to a single pathway. Their functionality ranged from mitochondrial maintenance (as in the case of $M R X 14, M R L P 1$ ) to biosynthesis of secondary metabolites such as INO2. This led to the hypothesis that the route used to attain resistance was dependent on the media type. Hence, if a certain strain was evolved in YM, it may not be resistant to the CFG in YPD. To confirm this hypothesis, semi quantitative growth assays of the evolved strains was carried out in different combinations of media conditions. The strains evolved in rich media did not confer cross resistance in minimal media. However, those evolved in minimal media and WYF concur cross resistance (Fig S2).

Many regions that underwent CNV were flanked by fragile sites, 12 out of 16 to be precise, mainly consisting of transposable elements (Lemoine et al. 2005) and one long regulatory ncRNA, ICR1 (Table 1). Fragile sites are defined as regions of the genome that make it difficult for the cell to undergo replication and sometimes result in chromosome breakage. Studies in other organisms such as $C$. elegans and human cells have shown evidence of DNA damage on exposure to CFG (Koller et al. 2012; Jacques et al. 2019). Past studies lack conclusive evidence to declare that Ty (yeast transposable elements) elements are involved in fragile site rearrangements resulting in CNV of certain regions. However, there is evidence to prove that these elements are involved in processes such as translocations, deletions, etc. (Dunham et al. 2002; Roeder \& Fink, 1980). Most of the regions undergoing increase in copy number and being flanked by Ty elements were found in S288c cells, evolved in the presence of $\mathrm{Cr} 41$. All the genes that contained non-synonymous SNPs or underwent CNV (S4 Table) were referred to as affected genes because they were affected by the Cr41 treatment (Fig 
3D). The regions that underwent CNV were large sections containing multiple genes from different pathways such as MAP kinase/ Hog1 pathway, mitochondrial genes, DNA damage repair pathways, spindle formation, metal transporters, cell wall and cell membrane.

\section{Transcriptome analysis revealed genes contributing to that glyphosate-tolerance}

To determine if there was a correlation between the genes that underwent changes in the ILES and those that are differentially expressed on exposure to $\mathrm{Cr} 41$, an RNAseq experiment was carried out. The RNA was extracted and sequenced from cells exposed to $\mathrm{Cr} 41$ in minimal media and media supplemented with WYF. The transcriptome analysis was performed on two strains, one of which was sensitive (S288c) to Cr41 exposure and the other, resistant (RM11). To decide on which two strains to consider for this study, we also took into account that the two strains had the most variation in the ILE study in terms of number of SNPs and the genes that underwent CNV. S288c, the sensitive strain treated with $\mathrm{Cr} 41$, had a much higher number of differentially expressed genes from the RNAseq (Fig 4A). In YM, 1100 genes (S5 Table) and 438 genes (S6 Table) in media supplemented with WYF were differentially expressed in S288c when exposed to $\mathrm{Cr} 41$ with a final concentration of $0.25 \%$ glyphosate. In RM11, only 58 (S7 Table) and 53 (S8 Table) genes were differentially expressed in YM and media supplemented with WYF, respectively. RM11 differentially expressed few genes in both conditions i.e., minimal media and media supplemented with WYF (Fig 4B) and had a higher correlation in terms of number of common genes, with the conditions under which the cells were exposed to Cr41. KEGG pathway enrichment analysis of the differentially expressed genes in \$288c revealed that a large number of these genes corresponded to pathways involved in cell cycle, meiosis, DNA replication and MAPK signaling pathways, especially in WYF (Fig 4C and 4D). Among many others, SED1 is one of the cell wall genes that are differentially regulated along with a few transposable elements in S288c, but no SNPs were found in the ILEs. The downregulated genes mainly associated with biosynthesis of secondary metabolites and amino acids in both minimal media and media supplemented with WYF. 


\section{S288c cells arrested in G1 on exposure to Cr41 but not pure glyphosate}

The RNAseq data showed upregulation of the expression of cell cycle regulator genes encoding proteins that are G1 phase regulators such as Rad53, Cdc28, Nrm1 and Swi4 (Bertoli et al. 2013). Increased expression of these genes suggested that cells were arresting in $\mathrm{G} 1$ on exposure to $\mathrm{Cr} 41$. To ascertain if it was the glyphosate itself or a cumulative effect of all the additives in CFG that caused cell cycle arrest, cells treated with $\mathrm{Cr} 41$ and pure glyphosate were subjected to flow cytometry. RM11 and S288c were grown to log phase and then the asynchronous populations were exposed to $0.25 \%$ pure glyphosate and $\mathrm{Cr} 41$ (Fig S4). Within 30 minutes of $\mathrm{Cr} 41$ exposure, $70 \%$ of the population arrested in $\mathrm{G} 1$ and remained the same over the course of 6 hours while the culture density was maintained consistently (Fig 5). This was not the case in the untreated and pure-glyphosate treated cells. In these two cases the populations were evenly distributed across the G1, G2, and S phases. RM11 cells did not show any changes in cell cycle with respect to the two treatments. It was only S288c, the Cr41 sensitive strain that underwent $\mathrm{G} 1$ arrest almost immediately on exposure to $\mathrm{Cr} 41$.

\section{sed1 1 mutants are highly sensitive to $\mathrm{Cr} 41$ exposure}

The genes commonly affected in the ILE study and differentially expressed in the RNAseq analysis belonged to various pathways, involving functions ranging from cell wall proteins, mitochondrial proteins, MAPK related proteins to meiosis (S4 Table). The BY4741 (also an S288c derived strain) knockout collection was used to test the growth phenotype of the different genes as representatives from some of these pathways. Five genes were selected for further characterization, all of which were associated with the cell wall or cell membrane. DET1 plays an integral role in intracellular sterol transport (Sullivan et al. 2009). PST1 encodes a GPI protein that works with Ecm33 to maintain cell wall integrity (Pardo et al. 2004). The presence of Ecm33 could be the cause for lack of sensitivity in pst1 mutants on exposure to $\mathrm{Cr} 41$. SED1 is a gene that is expressed as a major stress induced cell wall 
glycoprotein (Shimoi et al. 1998). FLO11 is another gene that encodes a GPI-anchored cell wall protein, whose transcription is regulated by the MAPK pathway (Rupp 1999). The flo11 1 mutants showed growth defects in minimal media. $V B A 5$ is a paralog of $V B A 3$. It codes for a plasma membrane protein that plays a role in amino acid uptake (Shimazu et al. 2012). Not all the knockout genes changed growth in response to $\mathrm{Cr} 41$ exposure as many of the genes may work in unison to have an overall effect on resistance to the treatment. Another possibility is the specific mutation could have a gain of function effect, which cannot be mimicked by using a knockout collection. One of the cell wall genes that had a definitive response was SED1. SED1 was found in both, the ILE data with its copy number doubled, and expression decreased by $1.475 \log 2$ fold in the transcriptome analysis. The sed $1 \Delta$ mutant was extremely sensitive to $\mathrm{Cr} 41$ exposure in both rich and minimal media (Fig 6A). In the S288c ILE strain that developed resistance to $\mathrm{Cr} 41$, SED1 underwent duplication in all media conditions, i.e., minimal media with and without WYF, and rich media. Down-regulation of SED1 gene in YM in S288c (sensitive strain) could contribute to the sensitivity of the cells to Cr41. No mutations were found in SED1 from the ILE sequencing, but there are several insertions and deletions found in RM11, YJM789 and AWRI1631 in reference to S288c (Fig 6B). Sed1 is a stress-induced structural GPI (glycosylphosphatidylinositol) cell wall glycoprotein (Shimoi et al.1998), hence its sensitivity to Cr41 due to the additives could be affecting the integrity of the cell.

\section{Calcofluor white (CFW) rescues growth inhibition of S288c caused by Cr41 exposure}

The analysis of affected genes in the evolved strains and the differentially expressed genes gave rise to a significant number of genes that are associated with the cell wall. To test if the cell wall played an important role in effectiveness of $\mathrm{Cr} 41$, one sensitive and one resistant strain that was treated with Cr41 was exposed to Calcofluor white (CFW). CFW is known for its property of inducing cell wall stress, as it is a chitin antagonist, and results in increased deposition of chitin making the cell walls thicker 
(Liesche, et al. 2015; Roncero and Duran 1985). A quantitative liquid growth assay was carried out using S288c and RM11. The growth of RM11 was inhibited by pure glyphosate (PG) and to a greater extent by Cr41. Treating the RM11 samples with CFW did not have a significant effect in YM for Cr41 or PG ( $p$ value $=0.19$ and 0.503$)$. In WYF, it was significant with $\mathrm{Cr} 41$ ( $p$-value $=0.011)$ but not PG ( $p$-value $=0.08)$. All strains closely related to S288c showed a higher sensitivity to pure glyphosate compare to Cr41 over the first 50 hours (Fig S5). Treating S288c cells in YM exposed to pure glyphosate with CFW did not have a significant effect ( $p$-value $=0.378)($ Fig 6C-E). However, CFW alleviated growth inhibition caused by Cr41 significantly $(p$-value $=0.024)$. Cells treated with CFW have increased cell wall volume by about $30 \%$ and the wall/cell ratio also increases significantly (Liesche et al. 2015). The increase in cell wall thickness could be the main contributor to the rescue of cells treated with $\mathrm{Cr} 41$. This indicated that the additives in $\mathrm{Cr} 41$, along with the glyphosate have a cumulative effect on the cell wall of sensitive strains, such as S288c, which was alleviated by CFW treatment. The increase in cell wall thickness on CFW treatment helps the cells withstand the effects of $\mathrm{Cr} 41$, possibly reducing the amount of $\mathrm{Cr} 41$ entering into the cell. However, treating cells exposed to only pure glyphosate with CFW does not result in alleviation of growth inhibition. This implies that CFW is not rescuing cells from the glyphosate in Cr41, but rather alleviating the effects of the additives on the cell wall.

\section{Discussion}

Glyphosate based herbicides are most commonly used around the world because of the specificity of glyphosate, in acting solely on the aromatic amino acid pathway, that is absent in humans and other higher eukaryotes. The additives and surfactants present in the commercial formulations are chosen due to their intrinsic inert and non-toxic properties. There are variations from one CFG to the other in terms of these additives, which results in different extents of effectiveness of the herbicide (Defarge et al. 2018). The ingredients that are added in CFG considered non-active, enhance the potency of glyphosate and were not inert, as seen by changes in the transcriptome. A crucial part is the study of 
all the changes occurring within the cell to make the herbicide more effective due to the addition of additives and surfactants. The additives in the CFG along with other functions, play a key role in the entry of the active ingredient into the cell. This is done by first compromising the first protective physical barrier that they come in contact with, the cell wall. The effectiveness varies based on the structure and composition of the cell wall along with the alleles of the different mannoproteins that the cell contains. When the concentrations and combinations of additives change, this also changes their cumulative effect on the cell. When multiple CFG were tested on different strains of yeast, this resulted in variation in their growth phenotype. The strains chosen for this study have been isolated from different environments as prior exposure was suspected to have an effect of the extent of tolerance to CFG. The agricultural isolates (RM11, AWRI1631) were more resistant to CFGs and glyphosate compared to the laboratory strain (S288c) and the clinical isolate (YJM789). The agricultural isolates may have come in contact with glyphosate or similar herbicides in the past, which could have led to development of resistance mechanisms based on prior exposure. The lab strain and clinical isolate are not in environments commonly exposed to CFG, making it less likely for them to have been exposed in the past, contributing to their sensitivity.

The genetic variation between these strains was used to gain a better understanding of the effects of the CFGs as a whole at the genome and transcriptome levels. In case of some stressors, adaptation can occur through a specific route depending on the regions of the genome most affected by the stressor. Response to CFG is a polygenic trait, as the different components of the herbicide act together to affect many genes that contribute to various pathways. Hence, adaptation can result from small modifications that occur in many different genes as a cumulative effect, as well as one particular change that could have caused a selective sweep and dominated the population. Large sections of the genome were found to have undergone CNV, many of which were flanked by Ty elements and ncRNAs. Regulatory elements have been shown to play an important role in causing elevated genetic variation 
that facilitates adaptation (McClintock 1984). S. cerevisiae has five families of LTR retrotransposons, and they comprise about 3\% of the entire genome (Garfinkel 2005). Mutations in certain DNA repair genes results in expression of common fragile sites, which when induced, often undergo translocations, deletions, duplications, etc. (Casper et al. 2002). The ILE strains contained mutations in genes involved in many pathways, some of which were contributors of DNA repair mechanisms (such as RAD53, UME6, and $M S H 3$ ) as well. Any of these alterations could have induced fragile site expression, in turn resulting in duplication of genes present between two Ty elements. Having the Ty elements in these regions provided the opportunity for these genes to undergo duplication, and those with a beneficial effect were retained as the population progressed. Over the course of serial passaging of these strains, the duplicated regions, along with the effects of SNPs in some of the genes, may have provided the advantage that the cells needed to overcome sensitivity to Cr41. To assess if there was an overarching contribution by a particular gene that underwent modification, or if it was the cumulative effect of many of the changes, some of the genes were tested using the knockout collection. This led to the observation that Sed1 is an important contributor to the cell's resistance of CFG.

The most drastic effects of glyphosate-based herbicides on the cell, apart from the aromatic amino acid pathway were on the cell wall. The cell wall is the mode of entry into the cell, and a vital structure in maintaining the osmotic integrity of the cell. Changing the combinations or concentrations of adjuvants in an herbicide alter the mode of entry of the active component into the cell, while also affecting the cell wall and many proteins involved in the transport of substances in and out of the cell. In our previous study, we have shown that proteins such as Dip5, the aspartic and glutamic acid permease (Rong-Mullins et al. 2017a) are involved in import of some glyphosate into the cell. Along with Dip5, there could be other proteins that are being affected. The effect of pleiotropic drug response genes is more evident in rich media. PDR5 is the most polymorphic gene in yeast (Guan et al. 2010). Pdr5 shares 96\% amino acid similarity between S288c and YJM789, whereas Pdr5 is 99.7\% similar between S288c 
and RM11, which may have led to the masking of its contribution in this study. Sed1 is a GPI-cell wall protein that is highly expressed when the cells are in stationary phase and is a required protein for cells in this phase when they are under stress (Shimoi et al. 1998). SED1 is not very highly conserved across strains, and a deletion n RM11 led to a similarity of only $87.87 \%$ between the S288c and RM11 alleles. Like Pdr5, the role of Sed1 appears to be dealing with the effects of the additives in Cr41. This can be said, as knockouts of both these genes are very sensitive on YPD which requires higher levels of Cr41 to induce growth inhibition. The MAPK pathway along with Hog1 genes, co-regulate genes involved in maintaining the integrity of the cell and cell wall. Several Hog1 MAP kinase genes (FUS3, STE5, BMH2, and AFR1) contained gene duplications in the evolved strains. This pathway is activated under conditions of hyperosmotic stress, and is usually accompanied by differential expression of various GPI-cell wall proteins. The CFG as a whole could be inducing hyperosmotic stress, resulting in the activation of the Hog1 MAP kinase pathway which could also contribute to arrest in G1 phase (Escoté, et al. 2004). The G1 arrest could be due to a combination of effects such as nutrients starvation and cell wall damage.

In this study, we highlight the importance of studying different CFG to gain a better understanding of all the pathways affected on exposure to $\mathrm{Cr} 41$, and to recognize the various non-target adaptation mechanisms. Higher eukaryotes have proteins that have structural and functional similarity to those found in yeast. As this herbicide is used so extensively on produce that is used for human consumption, it is important that we understand the effects of different chemicals we are being exposed to. Recent studies have shown the presence of glyphosate and its metabolites in urine samples in humans (Mills et al. 2017b; Parvez et al. 2018) though the levels of glyphosate humans are exposed to through food consumption is much lower than those used in this study to treat yeast cells. Without analyzing the effects at the molecular level, it is difficult to predict if there will be any long-term effects. Not seeing any immediate effects in humans, is not necessarily an indication that there would not be any effects on long-term exposure. Although human cells don't have cell walls, the trascriptome and ILE 
data indicate many other highly conserved pathways in the cell are affected. Hence, it is crucial to study the effects of different CFG to be able to regulate the use of additives and surfactants.

\section{Materials and Methods}

\section{Variations in growth phenotype}

A semi-quantitative growth assay was carried out on exposure of AWRI1631, RM11, YJM789, and S288c, to different formulations of glyphosate-based herbicides. The media used for the study was nutrient rich (YPD) and nutrient minimal (YM) solid media. The rich media is comprised of yeast extract, peptone and dextrose; as for the minimal media, it consists of yeast-nitrogen base and $2 \%$ dextrose. The minimal media was supplemented with aromatic amino acids, namely $20 \mu \mathrm{g} / \mathrm{ml}$ tryptophan (W), 30 $\mu \mathrm{g} / \mathrm{ml}$ tyrosine $(\mathrm{Y})$ and $50 \mu \mathrm{g} / \mathrm{ml}$ phenylalanine $(\mathrm{F})$. It was also supplemented with $100 \mu \mathrm{g} / \mathrm{ml}$ aspartic (D) acid for certain conditions. This assay was performed as previously described in (Rong-Mullins et al. 2017a), as follows. These were exposed to the glyphosate-based herbicides and the concentration of glyphosate was standardized to $1.0 \%$ in rich and $0.15 \%$ in minimal media respectively. The different formulations used were, Compare and save (CAS), WeedPro (WP), Super concentrated (SC), Credit 41 (Cr41) and pure glyphosate as a control.

Quantitative growth analysis was carried out using a TECAN M200, automatic plate reader (Rong-Mullins et al. 2017b). The growth of cells in liquid media was measured every 1 hour at 600nm under shaking conditions. Along with Credit41 and pure glyphosate, the cells were treated with 5mM calcofluor white, to measure the progression of their growth over 50 hours.

\section{Gene expression analysis}

RNA was extracted from S288c and RM11 cells grown in minimal media with and without supplementing WYF. The cells were treated with $0.25 \%$ Credit 41 for 90 minutes with 5 replicates of each 
condition. The samples were then washed, and the total RNA was extracted using hot phenol method (Ausubel et al. 1995). Paired end cDNA libraries were built using the RNA extracted using Epicenre's ScriptSeq Yeast kit. The sequencing was performed using 76bp paired end reads on the Illumina HiSeq platform resulting in 5- 8.5 million read pairs.

Salmon v0.13.1 was used to estimate the transcript level abundance from the RNA seq-read data (Patro et al. 2017). To do so, indexing of the S288c reference transcriptome was first carried out. This index was used by Salmon against each sample to generate quant.sf files containing the length, abundance in terms of Transcripts Per Million (TPM) and estimated number of reads for each transcript(https://www.ncbi.nIm.nih.gov/geo/query/acc.cgi?acc=GSE135473 secure token: arkxmokwdlufjir). The differential expression of genes between the two strains and each condition was calculated using DESeq2 v1.8.1. DESeq2 was also used to generate a sample to sample distance map (Fig S3). clusterProfiler v3.10.0 was used to carry out KEGG Pathway Enrichment Analysis, to identify the pathways involved, based on the clustering of genes.

\section{Flow cytometry}

To measure the cell cycle arrest on Credit41 and pure glyphosate exposure, S288c and RM11 cells were subjected to flow cytometry after exposure. The cultures were started and allowed to grow to mid-log phase from an overnight culture. Once the cells reached mid-log phase, they were treated with $0.25 \%$ Credit41 and pure glyphosate, in triplicate with and without supplementing WYF in minimal media. Cells were collected at multiple timepoints ( 0 mins, 30 mins, 90 mins, 3 hrs and 6 hrs) over the next 6 hours. The cells were maintained in log-phase throughout the collections, by replenishing with fresh media as needed. The cells were harvested and fixed in $70 \%$ ethanol for 2 days (Haase and Reed 2002). The cells were then washed and treated with RNAse solution for $8-12$ hours at $37^{\circ} \mathrm{C}$. The RNAse solution comprised of a mixture of $2 \mathrm{mg} / \mathrm{ml}$ RNAse $\mathrm{A}, 50 \mathrm{mM}$ Tris $\mathrm{pH} 8.0$, and $15 \mathrm{mM}$ sodium chloride that 
had been boiled for 15 minutes and cooled to room temperature. The cells were then treated with protease solution $\left(5 \mathrm{mg} / \mathrm{ml}\right.$ pepsin and $4.5 \mu \mathrm{l} / \mathrm{ml}$ conc $\mathrm{HCl}$ ) for 20 minutes at $37^{\circ} \mathrm{C}$. The cells were then stored in $50 \mathrm{mM}$ Tris pH 7.5 at $4^{\circ} \mathrm{C}$, until the day of the analysis. Right before flow cytometry analysis, the cells were sonicated at low levels to separate cells and avoid large clumps of cells. 50ul of the sonicated mixture was transferred into $1 \mathrm{ml}$ of $1 \mathrm{uM}$ Sytox Green in $50 \mathrm{ml}$ Tris $\mathrm{pH}$ 7.5. The cells were analyzed on a LSRFortessa, using the FITC channel. The results were analyzed, and the percentage of cells in each stage of the cell cycle was estimated using FCS Express 6.0. The analysis was carried out using the multi-cycle DNA histogram and '+S order=1 model' was selected based on the lowest Chisquare value.

\section{Whole-genome sequencing of In-Lab evolutions (ILEs)}

In-lab evolutions were carried out by exposing cells to Credit41 over $\sim 500$ generations, until a resistant population was isolated. Two sensitive (S288c and YJM789), and two resistant (RM11 and AWRI1631) strains were used for this study. The cells were evolved in minimal media with $0.25 \% \mathrm{Cr} 41$, with and without supplementing WYF. The cells evolved in rich media were treated with $1.0 \% \mathrm{Cr} 41$. A control group was evolved in media in the absence of Credit41 to account for mutations that occur due to the procedure, rather than the Credit41 treatment. A single colony was used to start a saturated overnight culture, that was then used as starter culture to inoculate the different conditions in triplicate. Each passage was grown 2-3 days in media till saturation. After which $1 \%$ of the culture was transferred into fresh media. The cells were subjected to 6 passages and tested for resistant populations by plating 10-fold dilutions on solid media. The resistant populations were streaked on plates to isolate single colonies. The resistant colonies were selected based on size and shape. They were then passaged for 2 passages in media lacking Credit41, to ensure the resistance did not rely solely on epigenetic mechanisms. After which they were tested on $\mathrm{Cr} 41$ containing media and re-streaked for single colonies. 
The genomic DNA was then extracted using the ThermoFischer 96 well genomic DNA extraction kit, and the phenol chloroform extraction technique was also used (Hirt 1967).

The whole-genome sequencing data for all 4 strains were aligned to S288c reference sequence (release R64-2-1) by creating an index and using gatk-4.1.1.0 to carry out the alignment. The controls set up in the experiment, that were evolved in the absence of Credit41 were used to eliminate strain specific variations. Samtools was used to convert the SAM files to BAM files, from which duplicates were removed. Using gatk HaplotypeCaller, variant calling was carried out to identify all the SNPs and generate vcf files. Only non-synonymous SNPs were used for the analysis. The analysis was carried out at the gene level, where a gene present in the control strains containing a SNP were automatically filtered out from the experimental samples. Using the generated vcf files, PCA plots were developed using pcadapt as described in (Hugh et al. 2019) (https://data.mendeley.com/datasets/ts5syfw38r/draft?a=e2170360-459e-4a8c-b1b3-d89f4b530fdf) (doi 10.17632/ts5syfw38r).

\section{Acknowledgements}

We thank Angela Lee for the yeast knockout collection. We would like to thank Kathy Brundage and the WVU Flow Cytometry Core (Fortessa S10 grant: OD016165), along with Ryan Percifield and the Genomics Core Facility for their services. This work was supported by NSF MCB-1614573 and WVU Senate Research Grant program. R-14-003. 


\section{References}

Aguilar-Uscanga, B., and J. M. François, 2003 A study of the yeast cell wall composition and structure in response to growth conditions and mode of cultivation. Lett. Appl. Microbiol.

Amrhein, N., B. Deus, P. Gehrke, and H. C. Steinrücken, 1980 The Site of the Inhibition of the Shikimate Pathway by Glyphosate. 66: 830-834.

Ausubel, F. M., R. Brent, R. E. Kingston, D. D. Moore, J. G. Seidman et al., 1995 Short protocols in molecular biology, 1995.

Bertoli, C., J. M. Skotheim, and R. A. M. De Bruin, 2013 Control of cell cycle transcription during G1 and S phases. Nat. Rev. Mol. Cell Biol.

Borneman, A. R., A. H. Forgan, I. S. Pretorius, and P. J. Chambers, 2008 Comparative genome analysis of a Saccharomyces cerevisiae wine strain, in FEMS Yeast Research,.

Casper, A. M., P. Nghiem, M. F. Arlt, and T. W. Glover, 2002 ATR regulates fragile site stability. Cell.

Defarge, N., J. Spiroux de Vendômois, and G. E. Séralini, 2018 Toxicity of formulants and heavy metals in glyphosate-based herbicides and other pesticides. Toxicol. Reports.

Defarge, N., E. Takács, V. L. Lozano, R. Mesnage, J. S. de Vendômois et al., 2016 Co-formulants in glyphosate-based herbicides disrupt aromatase activity in human cells below toxic levels. Int. J. Environ. Res. Public Health.

Duke, S. O., 2018 The history and current status of glyphosate. Pest Manag. Sci.

Duke, S. O., and S. B. Powles, 2008 Glyphosate: A once-in-a-century herbicide, in Pest Management Science,

Dunham, M. J., H. Badrane, T. Ferea, J. Adams, P. O. Brown et al., 2002 Characteristic genome rearrangements in experimental evolution of Saccharomyces cerevisiae. Proc. Natl. Acad. Sci. 
Engel, S. R., F. S. Dietrich, D. G. Fisk, G. Binkley, R. Balakrishnan et al., 2014 The Reference Genome Sequence of Saccharomyces cerevisiae: Then and Now. G3 Genes|Genomes|Genetics 4: 389-398.

Escoté, X., M. Zapater, J. Clotet, and F. Posas, 2004 Hog1 mediates cell-cycle arrest in G1 phase by the dual targeting of Sic1. Nat. Cell Biol.

García, R., J. M. Rodríguez-Peña, C. Bermejo, C. Nombela, and J. Arroyo, 2009 The high osmotic response and cell wall integrity pathways cooperate to regulate transcriptional responses to zymolyase-induced cell wall stress in Saccharomyces cerevisiae. J. Biol. Chem.

Garfinkel, D. J., 2005 Genome evolution mediated by Ty elements in Saccharomyces. Cytogenet. Genome Res.

Gasch, A. P., P. T. Spellman, C. M. Kao, O. Carmel-Harel, M. B. Eisen et al., 2000 Genomic Expression Programs in the Response of Yeast Cells to Environmental Changes. Mol. Biol. Cell.

De Groot, P. W. J., A. F. Ram, and F. M. Klis, 2005 Features and functions of covalently linked proteins in fungal cell walls. Fungal Genet. Biol.

Guan, W., H. Jiang, X. Guo, E. Mancera, L. Xu et al., 2010 Antagonistic changes in sensitivity to antifungal drugs by mutations of an important $A B C$ transporter gene in a fungal pathogen. PLoS One 5: e11309.

Haase, S. B., and S. I. Reed, 2002 Improved Flow Cytometric Analysis of the Budding Yeast Cell Cycle. Cell Cycle 1: 117-121.

Haderlie, L. C., J. M. Widholm, and F. W. Slife, 1977 Effect of Glyphosate on Carrot and Tobacco Cells. Plant Physiol.

Hatakeyama, R., M. Kamiya, T. Takahara, and T. Maeda, 2010 Endocytosis of the aspartic acid/glutamic acid transporter Dip5 is triggered by substrate-dependent recruitment of the Rsp5 ubiquitin ligase via the arrestin-like protein Aly2. Mol. Cell. Biol. 30: 5598-5607.

Hereward, J. P., J. A. Werth, D. F. Thornby, M. Keenan, B. S. Chauhan et al., 2018 Gene 
expression in response to glyphosate treatment in fleabane (Conyza bonariensis) - glyphosate death response and candidate resistance genes. Pest Manag. Sci.

Hirt, B., 1967 Selective extraction of polyoma DNA from infected mouse cell cultures. J. Mol. Biol. 26: 365-9.

Jacques, M. T., J. Bornhorst, M. V. Soares, T. Schwerdtle, S. Garcia et al., 2019 Reprotoxicity of glyphosate-based formulation in Caenorhabditis elegans is not due to the active ingredient only. Environ. Pollut.

Klis, F. M., P. Mol, K. Hellingwerf, and S. Brul, 2002 Dynamics of cell wall structure in Saccharomyces cerevisiae. FEMS Microbiol. Rev.

Koller, V. J., M. Fürhacker, A. Nersesyan, M. Mišík, M. Eisenbauer et al., 2012 Cytotoxic and DNA-damaging properties of glyphosate and Roundup in human-derived buccal epithelial cells. Arch. Toxicol.

Lemoine, F. J., N. P. Degtyareva, K. Lobachev, and T. D. Petes, 2005 Chromosomal translocations in yeast induced by low levels of DNA polymerase: A model for chromosome fragile sites. Cell.

Liesche, J., M. Marek, and T. Günther-Pomorski, 2015 Cell wall staining with Trypan blue enables quantitative analysis of morphological changes in yeast cells. Front. Microbiol.

Luu, K., E. Bazin, and M. G. B. Blum, 2017 pcadapt: an R package to perform genome scans for selection based on principal component analysis, in Molecular Ecology Resources,.

McClintock, B., 1984 The significance of responses of the genome to challenge. Science (80-. ).

McCusker, J. H., K. V. Clemons, D. A. Stevens, and R. W. Davis, 1994 Genetic characterization of pathogenic Saccharomyces cerevisiae isolates. Genetics.

Mesnage, R., B. Bernay, and G. E. Séralini, 2013 Ethoxylated adjuvants of glyphosate-based herbicides are active principles of human cell toxicity. Toxicology.

Mills, P. J., I. Kania-Korwel, J. Fagan, L. K. McEvoy, G. A. Laughlin et al., 2017 Excretion of the 
Herbicide Glyphosate in Older Adults Between 1993 and 2016. JAMA 318: 1610.

Moretti, M. L., R. Alárcon-Reverte, S. Pearce, S. Morran, and B. D. Hanson, 2017 Transcription of putative tonoplast transporters in response to glyphosate and paraquat stress in Conyza bonariensis and Conyza canadensis and selection of reference genes for qRT-PCR. PLoS One.

Mortimer, R. K., and J. R. Johnston, 1986 Genealogy of principal strains of the yeast genetic stock center. Genetics.

Mortimer, R. K., P. Romano, G. Suzzi, and M. Polsinelli, 1994 Genome renewal: A new phenomenon revealed from a genetic study of 43 strains of Saccharomyces cerevisiae derived from natural fermentation of grape musts. Yeast.

Nørskov, N. P., S. K. Jensen, and M. T. Sørensen, 2019 Robust and highly sensitive micro liquid chromatography-tandem mass spectrometry method for analyses of polar pesticides (glyphosate, aminomethylphosfonic acid, $\mathrm{N}$-acetyl glyphosate and $\mathrm{N}$-acetyl aminomethylphosfonic acid) in multiple biological matrices. J. Chromatogr. A 360343.

Nuoffer, C., P. Jenö, A. Conzelmann, and H. Riezman, 1991 Determinants for glycophospholipid anchoring of the Saccharomyces cerevisiae GAS1 protein to the plasma membrane. Mol. Cell. Biol.

Orlean, P., 2012 Architecture and biosynthesis of the Saccharomyces cerevisiae cell wall. Genetics 192: 775-818.

Orlean, P., 1997 Biogenesis of Yeast Wall and Surface Components, in The Molecular and Cellular Biology of the Yeast Saccharomyces: Cell Cycle \& Cell Biology,

Pardo, M., L. Monteoliva, P. Vázquez, R. Martínez, G. Molero et al., 2004 PST1 and ECM33 encode two yeast cell surface GPI proteins important for cell wall integrity. Microbiology.

Parvez, S., R. R. Gerona, C. Proctor, M. Friesen, J. L. Ashby et al., 2018 Glyphosate exposure in pregnancy and shortened gestational length: a prospective Indiana birth cohort study. Environ. Heal. 17: 23. 
Patro, R., G. Duggal, M. I. Love, R. A. Irizarry, and C. Kingsford, 2017 Salmon provides fast and bias-aware quantification of transcript expression. Nat. Methods 14: 417-419.

Powles, S. B., and C. Preston, 2006 Evolved Glyphosate Resistance in Plants: Biochemical and Genetic Basis of Resistance. Weed Technol. 20: 282-289.

Powles, S. B., and Q. Yu, 2010 Evolution in Action: Plants Resistant to Herbicides. Annu. Rev. Plant Biol.

Roeder, G. S., and G. R. Fink, 1980 DNA rearrangements associated with a transposable element in yeast. Cell.

Roncero, C., and A. Duran, 1985 Effect of Calcofluor White and Congo red on fungal cell wall morphogenesis: In vivo activation of chitin polymerization. J. Bacteriol.

Rong-Mullins, X., A. Ravishankar, K. A. McNeal, Z. R. Lonergan, A. C. Biega et al., 2017a Genetic variation in Dip5, an amino acid permease, and Pdr5, a multiple drug transporter, regulates glyphosate resistance in S. cerevisiae. PLoS One 12:.

Rong-Mullins, X., M. J. Winans, J. B. Lee, Z. R. Lonergan, V. A. Pilolli et al., 2017b Proteomic and genetic analysis of S. cerevisiae response to soluble copper leads to improvement of antimicrobial function of cellulosic copper nanoparticles. Metallomics 9: 1304-1315.

Rupp, S., 1999 MAP kinase and cAMP filamentation signaling pathways converge on the unusually large promoter of the yeast FLO11 gene. EMBO J.

Shimazu, M., T. Itaya, P. Pongcharoen, T. Sekito, M. Kawano-Kawada et al., 2012 Vba5p, a novel plasma membrane protein involved in amino acid uptake and drug sensitivity in Saccharomyces cerevisiae. Biosci. Biotechnol. Biochem.

Shimoi, H., H. Kitagaki, H. Ohmori, Y. limura, and K. Ito, 1998 Sed1p is a major cell wall protein of Saccharomyces cerevisiae in the stationary phase and is involved in lytic enzyme resistance. J. Bacteriol.

Song, G., B. J. Dickins, J. Demeter, S. Engel, J. Gallagher et al., 2015 AGAPE (Automated Genome Analysis PipelinE) for pan-genome analysis of Saccharomyces cerevisiae. PLoS One 10: 
e0120671.

Stewart, G. G., and G. G. Stewart, 2018 The Structure and Function of the Yeast Cell Wall, Plasma Membrane and Periplasm, in Brewing and Distilling Yeasts,.

Sullivan, D. P., A. Georgiev, and A. K. Menon, 2009 Tritium suicide selection identifies proteins involved in the uptake and intracellular transport of sterols in Saccharomyces cerevisiae. Eukaryot. Cell.

Tawfik, O. W., C. J. Papasian, A. Y. Dixon, and L. M. Potter, 1989 Saccharomyces cerevisiae pneumonia in a patient with acquired immune deficiency syndrome. J Clin Microbiol 27: 1689-1691.

Török, T., R. K. Mortimer, P. Romano, G. Suzzi, and M. Polsinelli, 1996 Quest for wine yeasts--an old story revisited. J. Ind. Microbiol. 17: 303-313.

Wenger, J. W., K. Schwartz, and G. Sherlock, 2010 Bulk segregant analysis by high-throughput sequencing reveals a novel xylose utilization gene from Saccharomyces cerevisiae. PLoS Genet.

Werner-Washburne, M., E. Braun, G. C. Johnston, and R. A. Singer, 1993 Stationary phase in the yeast Saccharomyces cerevisiae. Microbiol. Rev.

Yuan, J. S., P. J. Tranel, and C. N. Stewart, 2007 Non-target-site herbicide resistance: a family business. Trends Plant Sci.

Zlotnik, H., M. Pilar Fernandez, B. Bowers, and E. Cabib, 1984 Saccharomyces cerevisiae mannoproteins form an external cell wall layer that determines wall porosity. J. Bacteriol. 
Table 1. Regions with CNVs flanked by fragile sites in the ILEs

\begin{tabular}{|c|c|c|c|c|c|c|c|}
\hline Chromosome & Name & Type & start & end & width & Sample & Copy Number \\
\hline chrll & YBLCdelta7 & Ty1 LTR & 197016 & 197320 & 305 & AWRI-YM & CN2 \\
\hline chrll & YBLWsigma1 & Ty3 LTR & 197714 & 198054 & 341 & YJM789-WYF & CN2 \\
\hline chrIV & YDLCdelta1 & Ty1 LTR & 434423 & 434739 & 317 & AWRI-YM & CN2 \\
\hline chrIV & YDRCdelta7 & Ty1 LTR & 645502 & 645835 & 334 & GSY147-WYF & CN2 \\
\hline chrIV & YDRCdelta8 & Ty1 LTR & 651086 & 651419 & 334 & GSY147-WYF & CN2 \\
\hline chrIV & YDRCsigma1 & Ty3 LTR & 651420 & 651503 & 84 & GSY147-YPD & CN2 \\
\hline chrIV & YDRCdelta9 & Ty1 LTR & 651682 & 651980 & 299 & GSY147-YM & CN3 \\
\hline chrIV & YDRWsigma2 & Ty3 LTR & 668096 & 668436 & 341 & GSY147-WYF & CN2 \\
\hline chrIV & YDRWdelta10 & Ty1 LTR & 668543 & 668784 & 242 & GSY147-WYF & CN2 \\
\hline chrV & YERCdelta23 & Ty1 LTR & 492695 & 492833 & 139 & GSY147-YM & CN2 \\
\hline chrVIII & YHRCdelta16 & Ty1 LTR & 549306 & 549637 & 332 & AWRI-YPD & CN2 \\
\hline chrIX & ICR1 & IncRNA & 393884 & 397082 & 3199 & GSY147-WYF & CN2 \\
\hline
\end{tabular}




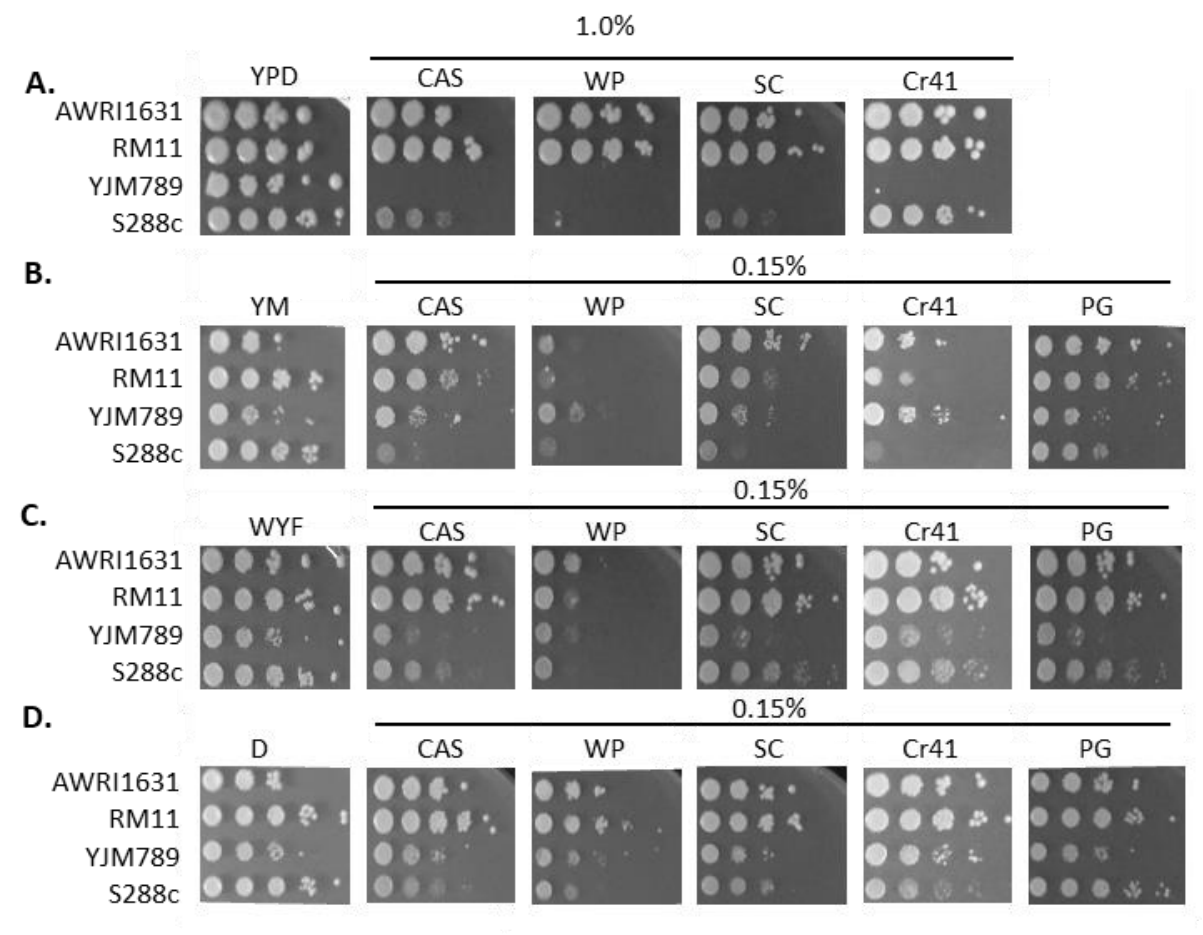

Fig1: Contribution of genetic variation of different yeast strains to glyphosate resistance. Serial dilutions of haploid AWRI1631, RM11, YJM789 and S288c were grown on the following media A. YPD with $1.0 \%$ of different formulations of glyphosate-based herbicides. B. YM with $0.15 \%$ of different formulations of glyphosate-based herbicides. C. YM+ aromatic amino acids (WYF) with $0.15 \%$ of different formulations of glyphosate-based herbicides and D. YM+ aspartic acid (D) with $0.15 \%$ of different formulations of glyphosate-based herbicides. Compare and save (CAS); WeedPro (WP); Super concentrated (SC); Credit 41 (Cr41) and pure glyphosate (PG). 
A.
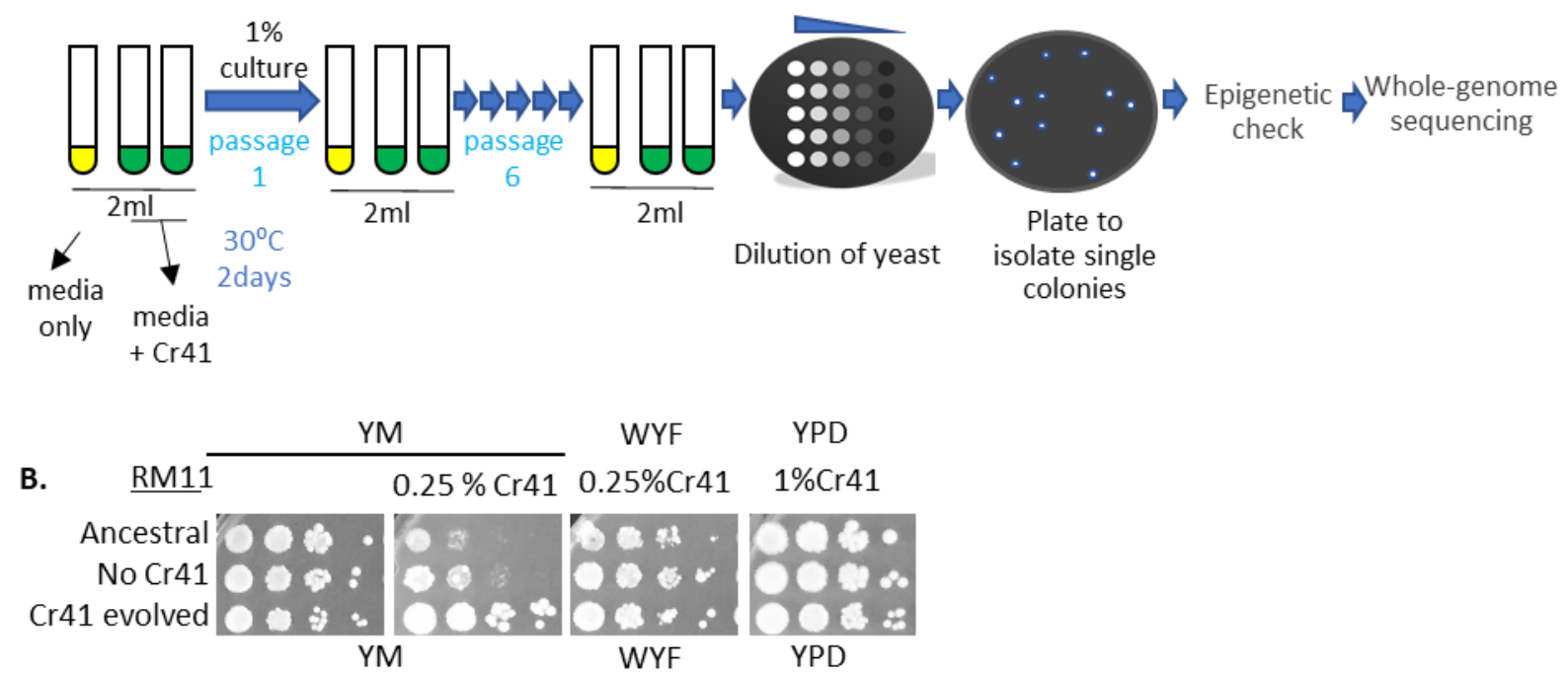

C. AWRI1631 $0.25 \% \mathrm{Cr} 41 \quad 0.25 \% \mathrm{Cr} 41 \quad 1 \% \mathrm{Cr} 41$

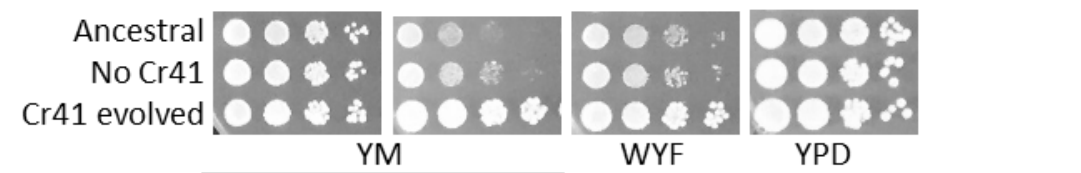

D. $\quad \underline{\mathrm{S} 288 \mathrm{c}} \quad 0.25 \% \mathrm{Cr} 41 \quad 0.25 \% \mathrm{Cr} 41 \quad 1 \% \mathrm{Cr} 41$

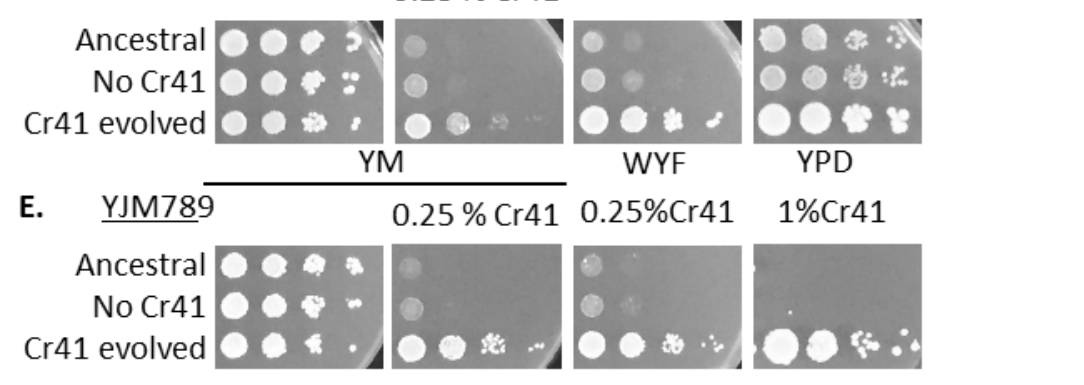

Figure 2: Selection of strains resistant to commercial formulations of glyphosate, Credit41 by In-lab evolutions (ILEs). A. Outline of In-lab evolutions (ILEs) methodology: The cells are grown in media with and without Credit 41 for 2 days at $30^{\circ} \mathrm{C}$ on the shaker. $1 \%$ of the culture was transferred to fresh media for 6 passages. Serial dilutions of the cultures of the sixth passage were plated on solid media. The resistant lines were plated on solid media to isolate single colonies. The genomic DNA was extracted and submitted for Illumina sequencing. B. Serial dilutions of haploid RM11, strain evolved without $\mathrm{Cr} 41$ and strain evolved with $\mathrm{Cr} 41$, on media with $\mathrm{Cr} 41$. C. Serial dilutions of haploid AWRI1631, strain evolved without $\mathrm{Cr} 41$ and strain evolved with $\mathrm{Cr} 41$ on media with $\mathrm{Cr} 41$. D. Serial dilutions of haploid S288c, strain evolved without $\mathrm{Cr} 41$ and strain evolved with $\mathrm{Cr} 41$ on media with $\mathrm{Cr} 41$. E. Serial dilutions of haploid YJM789, strain evolved without $\mathrm{Cr} 41$ and strain evolved with $\mathrm{Cr} 41$ on media with $\mathrm{Cr} 41$. 
A.

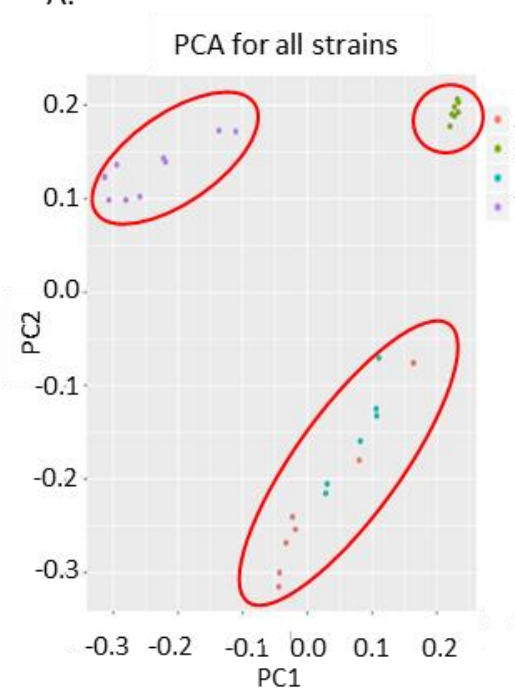

B.
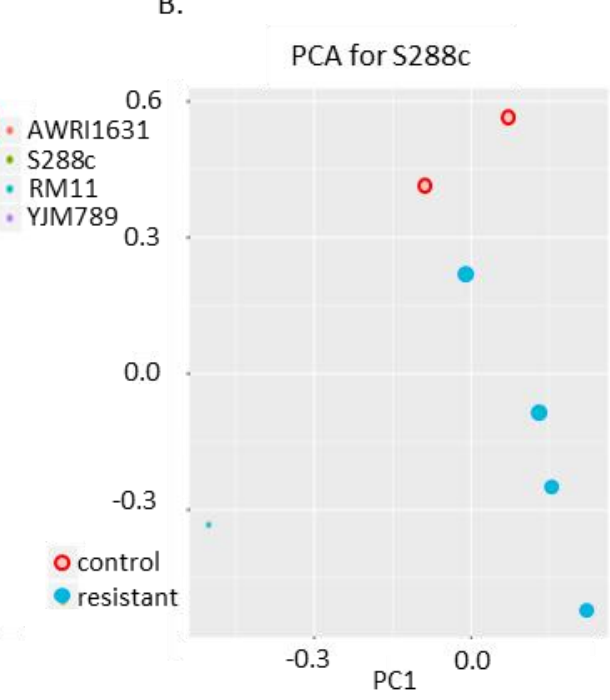

C.

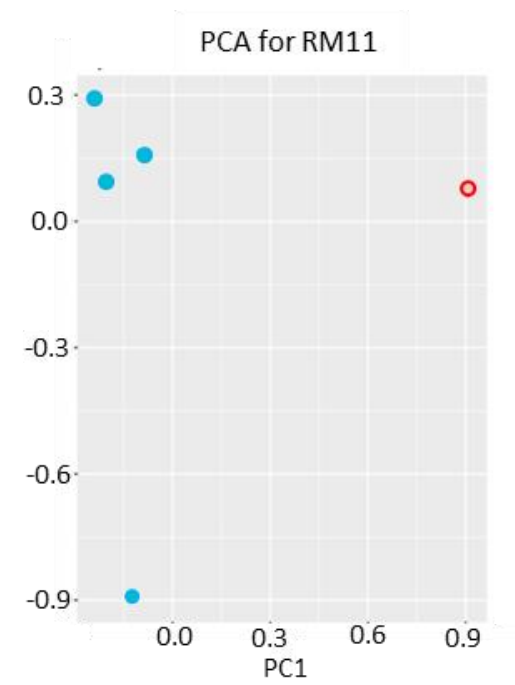

D.

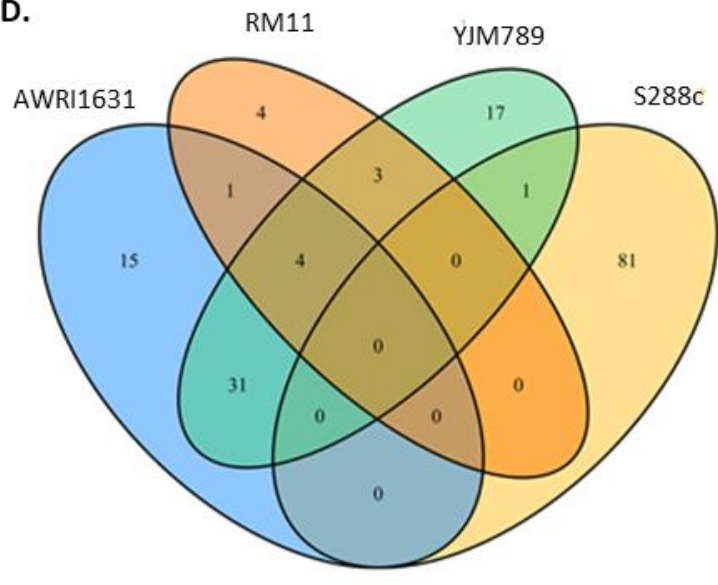

Figure 3: Whole-genome analysis of In-lab evolutions (ILEs). A. PCA of SNPs of various samples from all 4 strains, AWRI1631, S288c, RM11, and YJM789. B. PCA of SNPs identified among the S288c ILE samples C. PCA of SNPs identified among the RM11 ILE samples. D. Venn-diagram representing all the affected genes i.e., those that contained CNV, as well as those that accumulated SNPs, in each strain. 


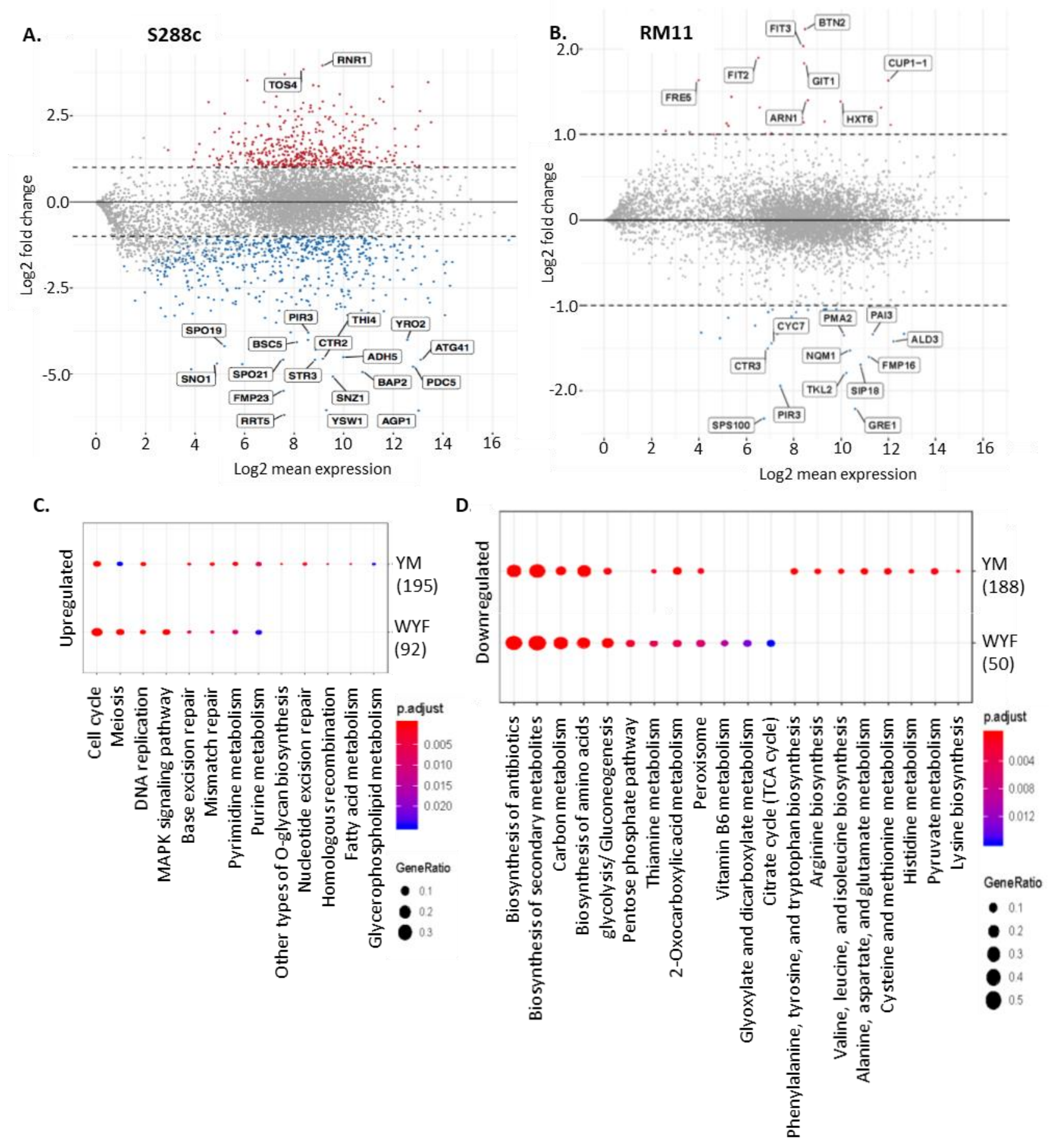

Fig 4 Gene expression analysis of S288c and RM11 on exposure to glyphosate (Credit41) in minimal media A. Dot-plot of significantly up and down-regulated genes in S288c and B. in RM11. C. KEGG Pathway Enrichment analysis of upregulated genes in S288c, identifying the different pathways involved. D. KEGG Pathway Enrichment analysis of downregulated genes in S288c, identifying the different pathways involved. 


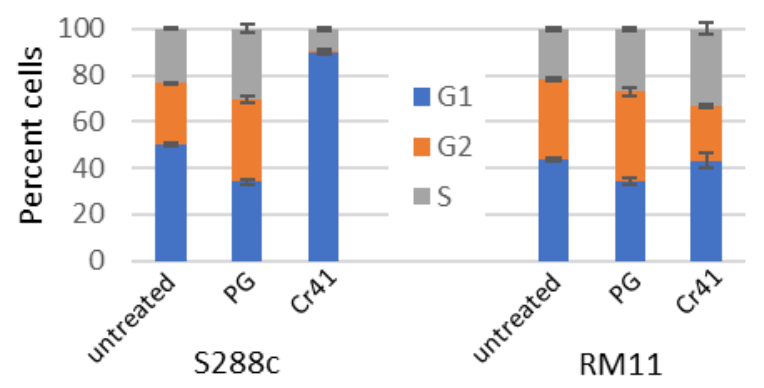

Figure 5. Cell cycle distribution on treatment with glyphosate and commercial glyphosate formulation Distribution of the cell cycle stages of S288c and RM11 cells on exposure to Credit41 (Cr41) and pure glyphosate (PG) for six hours. 


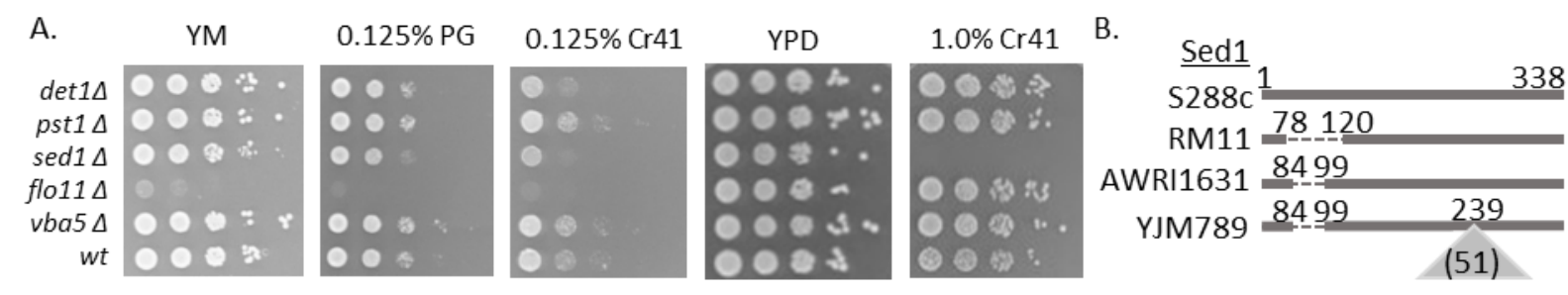

C.

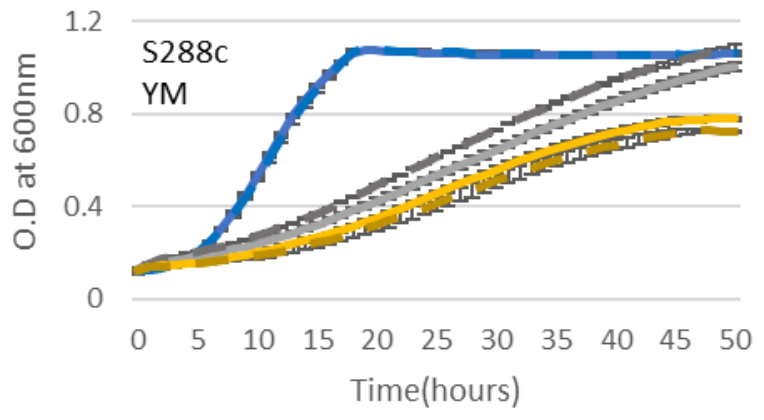

E.

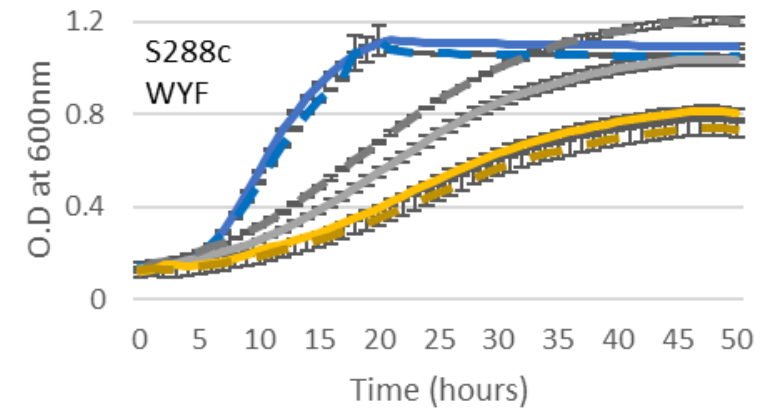

D.

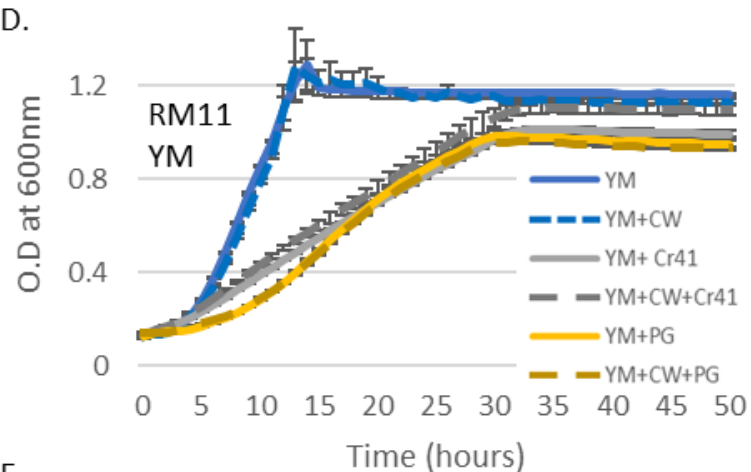

F.

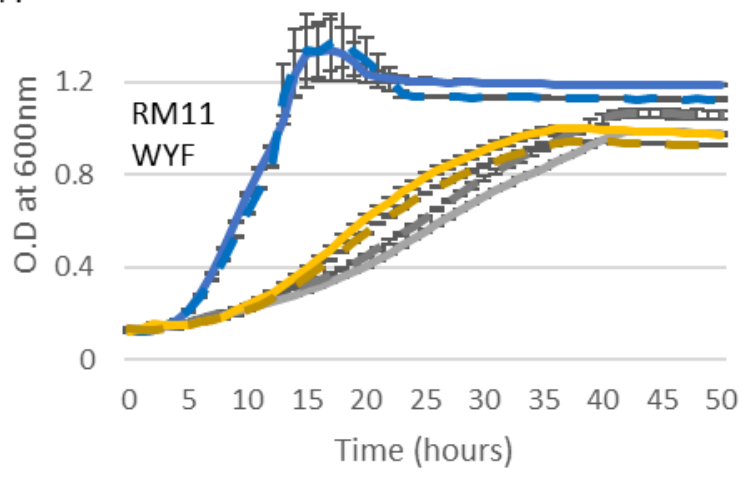

Fig 6: Growth assay on exposure to calcofluor white and glyphosate-based herbicides. A. Semiquantitative growth assay of cell wall mutants. Cells were grown for three days. B. Protein alignment of Sed1. Deletions in reference to the S288c are a dashed line and insertions are denoted by a triangle. Amino acid numbers are in reference to $\mathrm{S} 288 \mathrm{c}$ with the number of inserted amino acids in parentheses. Quantitative growth assays of cells grown in minimal media when exposure to calcofluor white (CW), Credit41 (Cr41) and pure glyphosate (PG). OD 600 was measure for 50 hours in an automatic plate reader. C. S288c in YM D. RM11 in YM. Yeast were grown in minimal media supplemented with tryptophan (W), tyrosine (Y), phenylalanine (F). E. S288c in WYF. F. RM11 in WYF. 


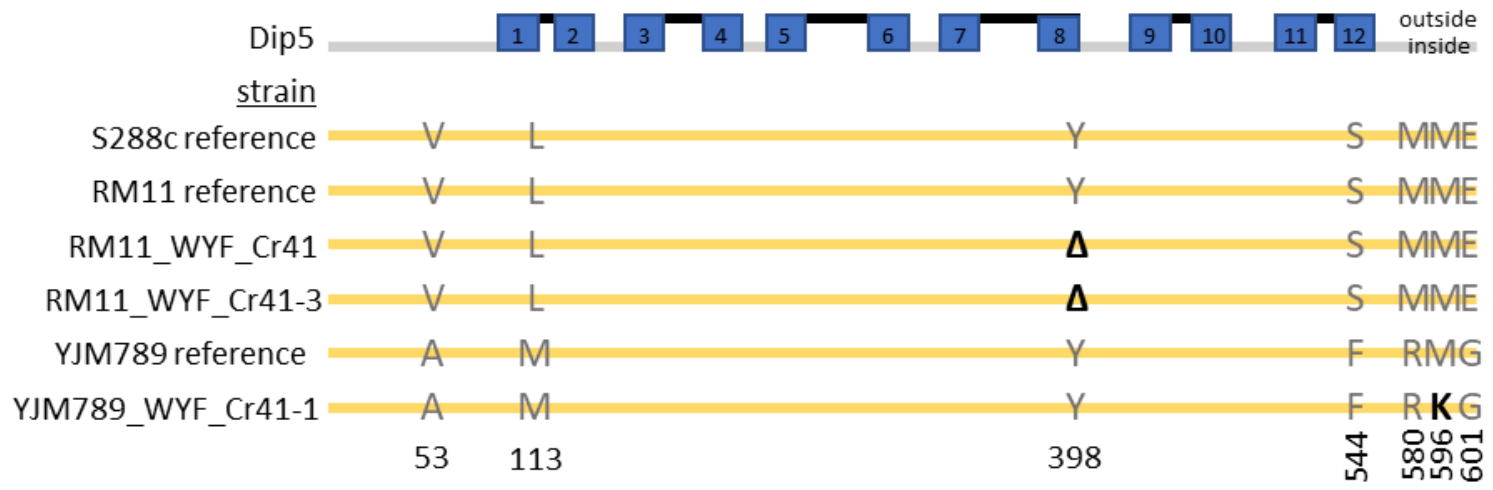

S1 Fig: Alignment of ILE strains containing mutations in DIP5. Three of the ILE strains contained mutations in the DIP5 gene. These were aligned to highlight all the polymorphisms in grey and mutations in bold with reference to the $\$ 288 \mathrm{c}$ and to each respective reference genome. The protein topology diagramed at the top is represented by black lines as the extracellular sequence and grey is the intercellular sequence. The blue boxes were predicted by TMHMM to be transmembrane domains (TM). The sequences for each TM of Dip5 ${ }^{\text {S288c }}$ are as follows TM1 91-113, TM2 120-142, TM3 157-179, TM4 200-219, TM5 234-253, TM6 286-308, TM7 323-345, TM8 376-398, TM9 423-445, TM10 455-477, TM11 500-522, and TM12 532-551. 
A.

\begin{tabular}{|c|c|c|}
\hline$\underline{\mathrm{S} 288 \mathrm{C}}$ & YPD & \\
\hline $\mathrm{NE}$ & $\mathrm{OO}^{\circ}$ & \\
\hline YM-cont & 00 क : & \\
\hline YM-2-Cr41 & 00 * & \\
\hline YM-3-Cr41 & $008:$ & . \\
\hline WYF-cont & 00 \% & $\bullet$ \\
\hline WYF-3-Cr41 & $00 \%:$ & . \\
\hline WYF-2-Cr41 & $00=$ & \\
\hline YPD-1-Cr41 & $008=$ & \\
\hline
\end{tabular}

C.

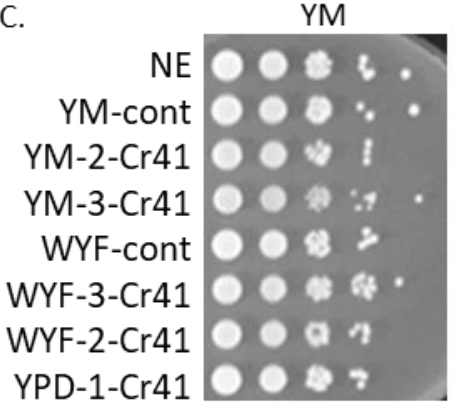

E.

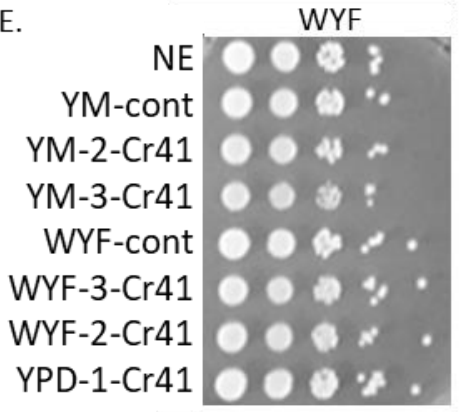

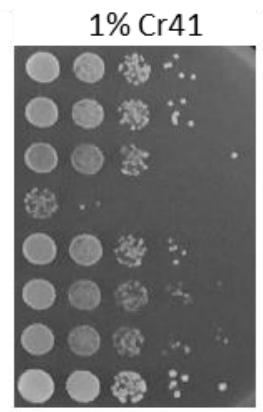

$0.25 \% \mathrm{Cr} 41$

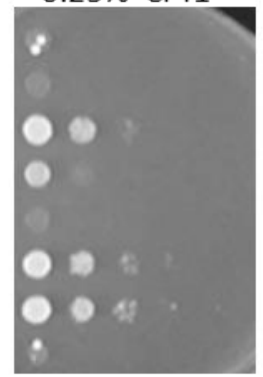

$0.25 \% \mathrm{Cr} 41$

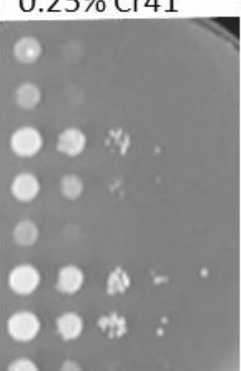

B.

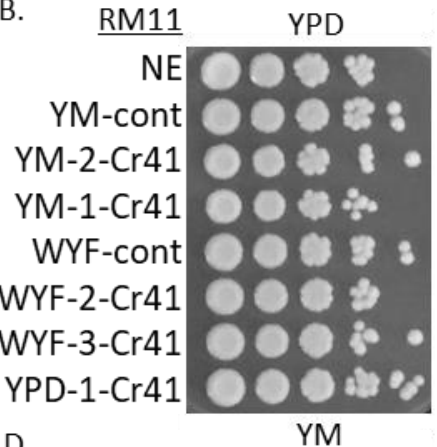

$D$.

YM

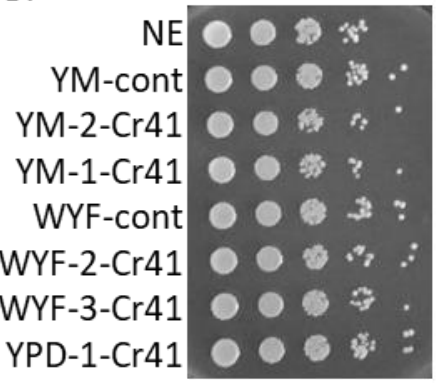

F. WYF

$\mathrm{NEO} O \mathrm{O}$ is

YM-cont $\mathrm{OO} O$ \&

YM-2-Cr410 0

YM-1-Cr41 $00 \div:$

WYF-cont $00 \%$ :

WYF-2-Cr41000 $\Rightarrow$

WYF-3-Cr41 000 a $\because$

YPD-1-Cr41 000 :

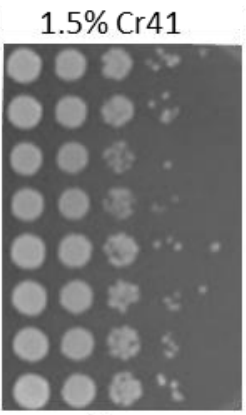

$0.25 \% \mathrm{Cr} 41$

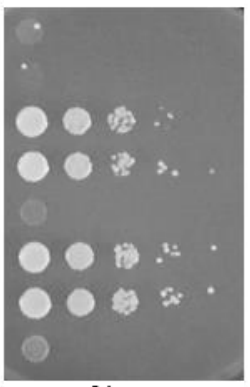

$0.25 \% \mathrm{Cr} 41$

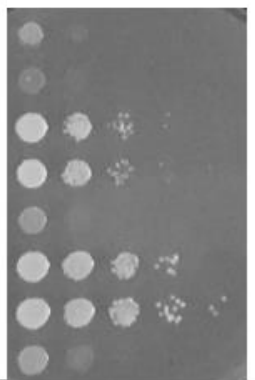

S2 Fig: Cross resistance was conferred in strains evolved in minimal media and WYF. A. Strains evolved under different conditions were tested for resistance in rich media with Cr41 for S288c and B. RM11. C. Strains evolved under different conditions were tested for resistance in minimal media with $\mathrm{Cr} 41$ in S288c and D. RM11. E. Strains evolved under different conditions were tested for resistance in minimal media supplemented with WYF and treated with $\mathrm{Cr} 41$ in S288c and F. RM11. 

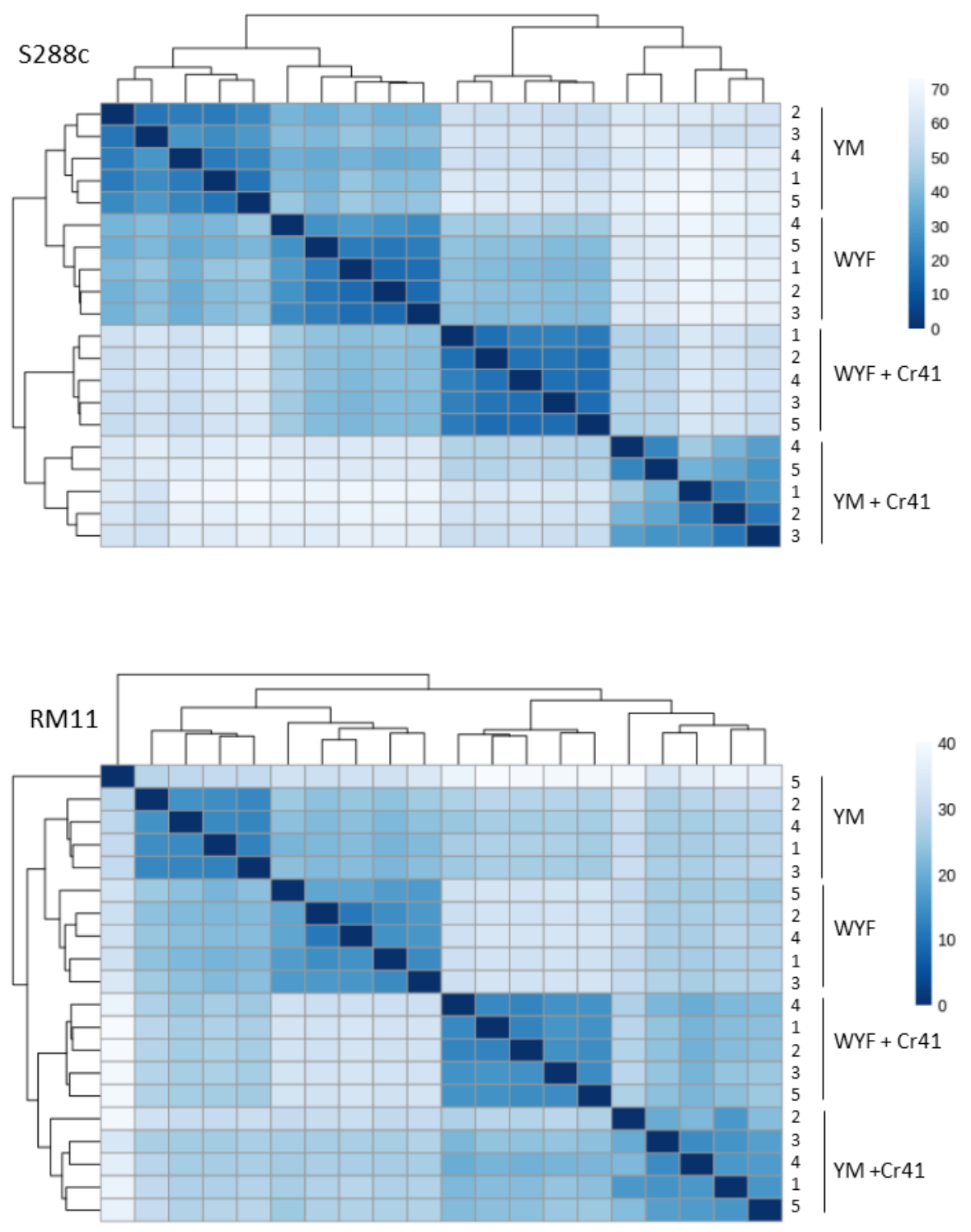

S3 Fig: Sample to sample distance estimation using DESeq2. The distance map shows clustering of biological replicates of RNAseq samples for A. S288c and B. RM11. This describes that the variation observed is based on $\mathrm{Cr} 41$ treatment and that the biological replicates can be used to collapse the data between the replicates. 
A. 120

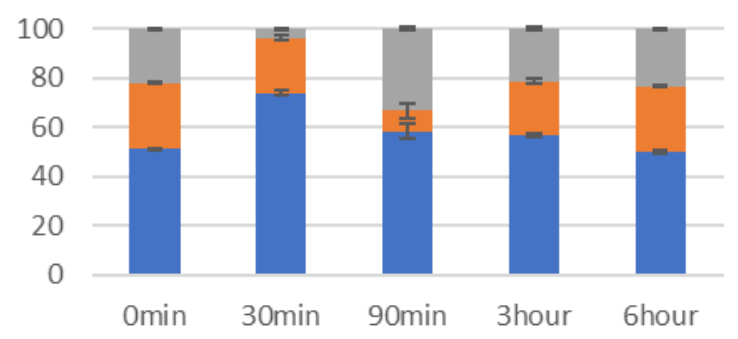

- G1 G2 as S288c-untreated

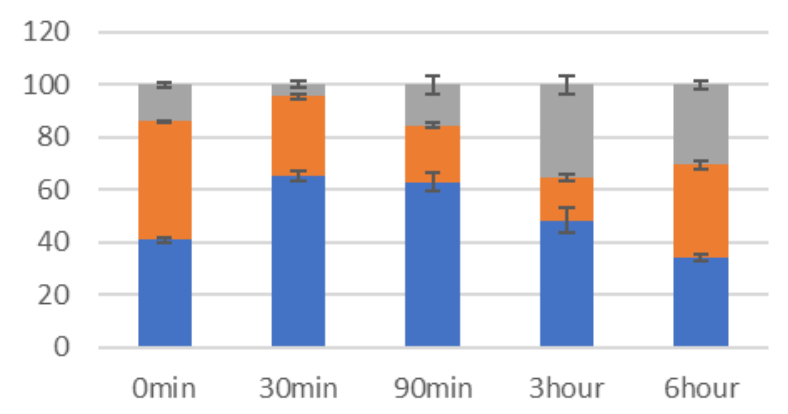

- G1 G2 GS S288c- PG treated

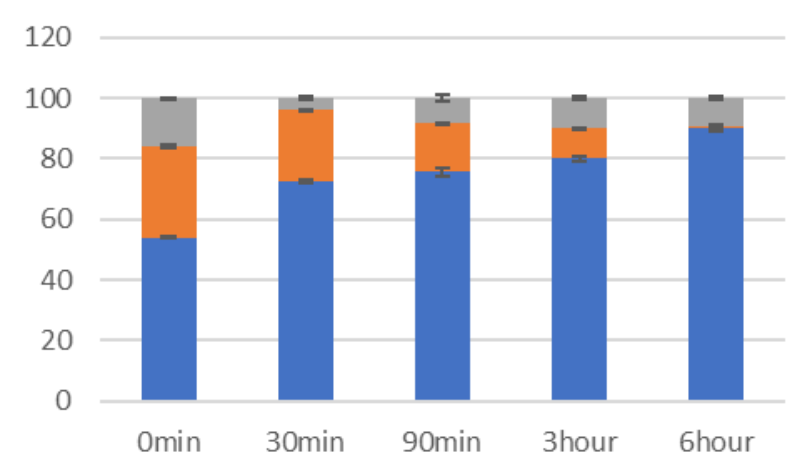

- G1 G2 G S288c- Cr41 treated
B. 120

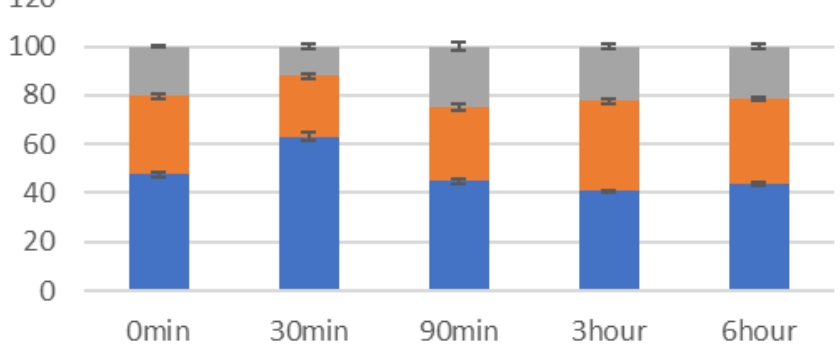

a 1 G2 $\square \mathrm{S}$ RM11- untreated

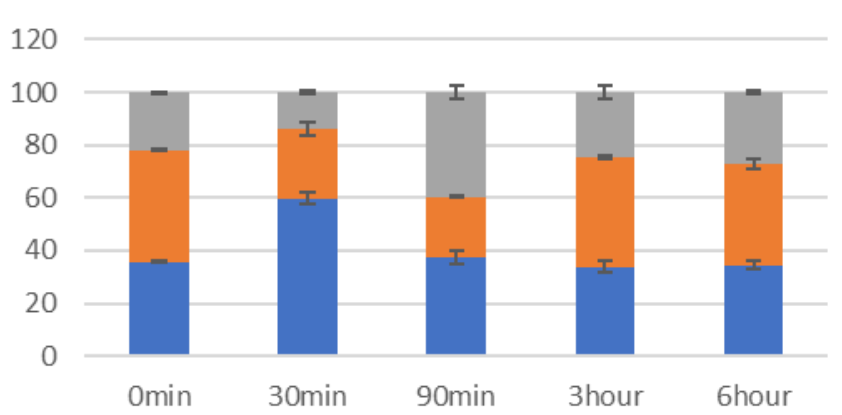

- G1 G2 $\square \mathrm{S}$ RM11-PG treated

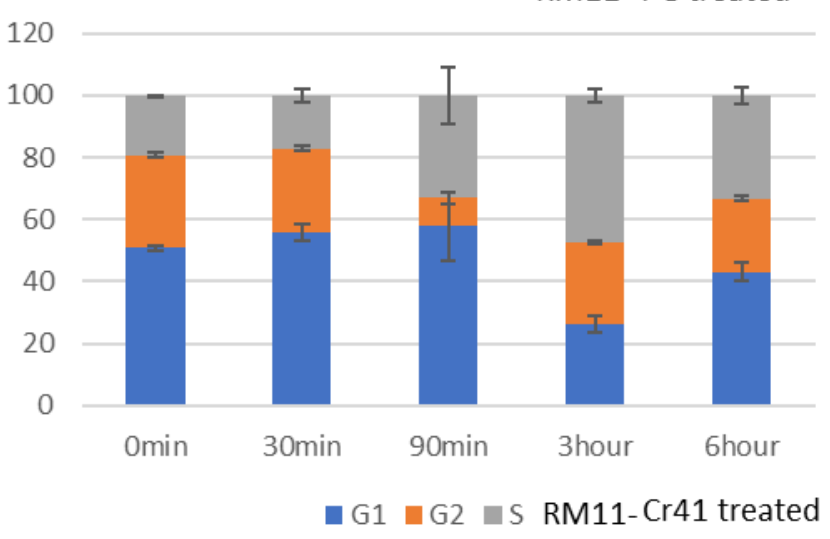

\section{S4 Fig: Flow cytometry of S288c strains show arrest in G1 phase of the cell cycle on treatment with}

$\mathrm{Cr} 41$. A. Cell cycle distribution of $\mathrm{S} 288 \mathrm{c}$ shows arrest in case of treatment with $\mathrm{Cr} 41$ but no arrest when treated with pure glyphosate B. Cell cycle distribution of RM11 shows no effect on treatment with $\mathrm{Cr} 41$. 


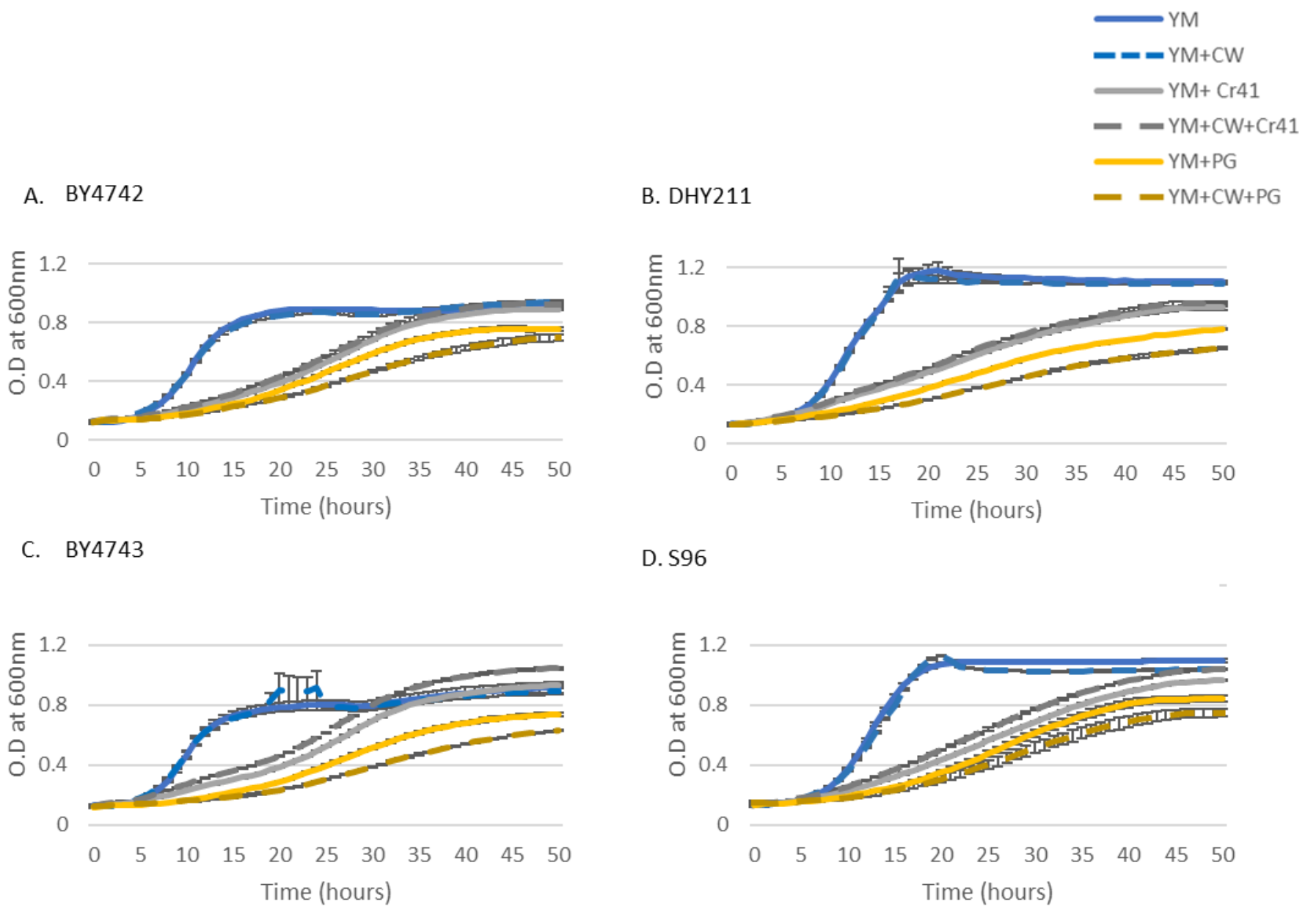

S5 Fig: Pure glyphosate inhibits growth to a greater extent than Cr41 only in strains closely related to S288c. S288c related cells were grown in minimal media, their growth was measured on exposure to calcofluor white (CW), Credit41 (Cr41) and pure glyphosate (PG) A. BY4742 B. DHY211 C. BY4743 D. S96 


\section{Supplemental Online resources}

Please use the links below to access the supplemental tables

https://drive.google.com/open?id=1-4b0W06MQG3SnAu1t-Tz0GaoTuZ4HIRY 


\title{
CHAPTER 5: MITOCHONDRIAL METABOLISM IS CRUCIAL IN RESPONSE AND RESISTANCE MECHANISMS OF SACCHAROMYCES CEREVISIAE ON EXPOSURE TO A GLYPHOSATE-BASED HERBICIDE
}

\author{
Paper submitted to Journal of Environmental Pollution \\ Apoorva Ravishankar, Jonathan R. Cumming, and Jennifer E.G. Gallagher*
}

\begin{abstract}
Glyphosate-based herbicides, the most extensively used herbicides in the world, are available in numerous forms of commercial formulations with varying additives and adjuvants. Here, we study the effects of one such formulation, Credit41, in two genetically diverse yeast strains. A quantitative trait loci (QTL) analysis between a sensitive laboratory strain and a resistant strain linked mitochondrial function to Credit41 resistance. Two genes encoding mitochondrial proteins identified through the QTL analysis were HFA1, a gene that encodes a mitochondrial acetyl CoA carboxylase, and $A A C 3$, which encodes a mitochondrial inner membrane ATP/ADP translocator. Further analysis of previously studied whole genome sequencing data of four yeast strains showed that, although each strain uses varying routes to attain resistance, most of them had duplications of mitochondrial proteins. One of the most well-studied functions of the mitochondria is the assembly of Fe-S clusters. In the current study, the expression of iron transporters in the transcriptome increased in cells resistant to Credit41. The levels of iron within the cell also increased in cells exposed to Credit41, but not pure glyphosate. Hence, the additives in commercial formulations have a significant contribution to the negative effects of these commercial formulations.
\end{abstract}


Keywords: Saccharomyces cerevisiae, glyphosate, mitochondria, iron-levels, QTL analysis

\section{Introduction}

The ubiquitous use of glyphosate-based herbicides around the world has led to extensive research of the effects of glyphosate on plants, bacteria and higher eukaryotes. Decades of research has been carried out on herbicide-resistance, mainly focusing on glyphosate. However, it is only in the past few years that the potential importance of the study of commercial formulations of these herbicides, along with their additives has been acknowledged. The aromatic amino acid pathway is the canonical target of glyphosate. The additives, although claimed to be neutral (Cox and Surgan 2006), when combined have a synergistic effect. Evaluating genetic variation in glyphosate-based herbicide resistance is one approach to address the toxic effects of these additives. The variation in response lies not only in the genetic make-up of the organism itself, but also in the variation in components and the concentrations of the adjuvants and surfactants added to the herbicides with the same active ingredient. Recent studies have shown that the toxicity of commercial formulations of glyphosate (CFG) is not due to the principal ingredient, but the additives in the various formulations (Jacques et al. 2019; Mesnage et al. 2015).

Saccharomyces cerevisiae is used extensively in the toxicogenomics study of various chemicals. Its benefits include a unique combination of highly conserved mechanisms of adaptation, tolerance and resistance related to higher eukaryotes (Parsons et al. 2003; dos Santos et al. 2012), along with the broad spectrum of variation that lies within the Saccharomyces species at the genome level. The environment from which the strains are isolated has a significant 
influence on this genetic variation between the strains (Landry et al. 2006; Lee et al. 2019;

Smith and Kruglyak 2008). The two strains used throughout this study were S288c and RM11.

RM11 is a Californian vineyard strain that was isolated from a vineyard in 1993 (Mortimer et al. 1994). As glyphosate-based herbicides were introduced in the 1970s it is likely that this strain was exposed to this stressor, and has subsequently developed adaptation mechanisms, as it is resistant to most CFG. S288c is a laboratory strain that that is sensitive to CFG (Rong-Mullins et al. 2017a).

In this study, the variation in phenotype and genotype between RM11 and S288c on Credit41 (Cr41) exposure was used to perform quantitative trait loci (QTL) analysis. The QTL was used to identify loci that contribute to differential $\mathrm{Cr} 41$ phenotypes. We found loci containing genes encoding mitochondrial proteins involved in mitochondrial fatty acid biosynthesis.

Transcriptome analysis of these strains revealed increased levels of metal transporters that are crucial in the transport of iron and copper in the resistant strain. The whole genome sequencing data of cells that adapted to $\mathrm{Cr} 41$ exposure were analyzed to find that numerous genes encoding mitochondrial proteins underwent duplication. These data imply that the mitochondria contribute to the resistant phenotype of cells exposed to this environmental stressor. Iron is an essential cofactor for numerous reactions within the cell. It is found mainly in cytochromes and iron-sulfur (Fe-S) clusters. It is the mitochondria that are mainly responsible for the biosynthesis of Fe-S clusters and its transport to the cytosol (Foury and Talibi 2001; Mühlenhoff and Lill 2000). This led to the further investigation of the varying levels of metal import into the cell in the presence of $\mathrm{Cr} 41$, and the differences exhibited in comparison to pure glyphosate. A marginally significant increase in iron levels in resistant cells (RM11) was 
found with Credit41 exposure, which may contribute to its resistant phenotype. The increased levels of iron transport were specific to the commercial formulation, $\mathrm{Cr} 41$, and not pure glyphosate, indicating that the effects observed were not a result of the principal component, but the additives and their collaborative effect.

\section{Materials and Methods}

\section{Variations in growth phenotype}

S288c and RM11 mutants of the candidate genes in the QTL were used to carry out a semiquantitative growth assay on exposure to $0.15 \% \mathrm{Cr} 41$. The solid media used for the study was minimal media (YM), minimal media supplemented with aromatic amino acids (tryptophan (W), tyrosine (Y), phenylalanine (F)) and minimal media supplemented with aspartic acid (D). The minimal media contains yeast-nitrogen base and $2 \%$ dextrose. The minimal media was supplemented with aromatic amino acids, $20 \mu \mathrm{g} / \mathrm{ml} \mathrm{W}, 30 \mu \mathrm{g} / \mathrm{ml} \mathrm{Y}$ and $50 \mu \mathrm{g} / \mathrm{ml} \mathrm{F}$. It was also supplemented with $100 \mu \mathrm{g} / \mathrm{ml} \mathrm{D}$ for certain conditions. This assay was performed as previously described in (Rong-Mullins, Ravishankar et al. 2017). The assay was repeated with the RM11 mutants at a higher concentration of $0.275 \% \mathrm{Cr} 41$, as RM11 is tolerant to much higher concentrations of $\mathrm{Cr} 41$. Double mutants in the RM11 background of the candidate genes were also tested in this manner for sensitivity to $\mathrm{Cr} 41$ exposure.

\section{Genetic Linkage Mapping}

Quantitative trait loci (QTL) analysis was performed using a recombinant segregant collection constructed from a cross between S288c and RM11 (Bloom et al. 2013). The yeast were grown 
in two conditions, minimal media supplemented with $0.25 \%$ Credit41 (Cr41) and minimal media supplemented with WYF and $0.25 \%$ Credit41 (Rong-Mullins, Ravishankar et al. 2017). The QTL analysis was performed using R-qtl as described in (Bloom et al. 2013).

\section{Gene expression analysis}

RNA was extracted from 5 biological replicates of S288c and RM11 cells exposed to Cr41 for 90 minutes in minimal media and media supplemented with WYF (Ravishankar et al. 2019). Salmon v0.13.1 was used to estimate transcript levels followed by DESeq2 v1.8.1 to carry out differential expression analysis. ClusterProfiler v3.10.0 was then used to carry out KEGG pathway enrichment analysis.

(https://www.ncbi.nlm.nih.gov/geo/query/acc.cgi?acc=GSE135473 secure token: arkxmokwdlufjir).

\section{Whole-genome sequencing of In-Lab evolutions (ILEs)}

Four strains (S288c, YJM789, AWRI1631 and RM11) were exposed to Cr41 for 6 passages in rich and minimal media. The genomic DNA was extracted and sequenced (Ravishankar et al. 2019) (https://data.mendeley.com/datasets/ts5syfw38r/draft?a=e2170360-459e-4a8c-b1b3d89f4b530fdf) (doi 10.17632/ts5syfw38r). To analyze and represent the commonalities between the mitochondrial proteins and the genes altered in the ILEs, Venny 2.0 was used

(Oliveros, J.C. (2007-2015) Venny. An interactive tool for comparing lists with Venn's diagrams. https://bioinfogp.cnb.csic.es/tools/venny/index.html)

\section{Element Analysis using ICP}


S288c and RM11 cells were grown in minimal media with and without $0.25 \% \mathrm{Cr} 41$ or pure glyphosate (PG) treatment. Five replicates of each sample were analyzed. The analysis was carried out as described in Pupo et al. (2019). Appropriate dilutions were made to measure the different amounts of each element being tested and normalized accordingly. The 10 OD units of cells collected were resuspended in $5 \mathrm{ml}$ to carryout extraction using hydrogen peroxide and concentrated nitric acid. It was then further diluted 2-fold to measure potassium, magnesium and phosphorous. For this study we measured the levels of iron, zinc, phosphorous, magnesium, calcium and potassium. The Agilent (5110 ICP, Agilent, Santa Clara, CA, USA) was used to measure the amounts of each element at the following wavelengths: Ca: 393.366, Fe: 238.204, Mg: 279.553, Na: 589.592, P: 213.618, and Zn: 206.200

\section{Results}

\section{Genetic linkage analysis of $\mathrm{Cr} 41$ response reveals involvement of mitochondrial genes}

Genetic variation between different yeast strains leads to variation in their tolerance to commercial formulations of glyphosate (Rong-Mullins, Ravishankar et al. 2017). To map the genes that contribute to their differential abilities in combating growth inhibition caused by CFG, a recombinant segregant mapping collection from S288c (sensitive strain) and RM11 (resistant strain) were used (Bloom et al. 2013). The segregant collection was tested in minimal media (YM) to identify loci contributing to the CFG resistance of RM11. The collection was also tested in YM supplemented with the aromatic amino acids (WYF). For the sake of this study, we chose to use $\mathrm{Cr} 41$ (a representative CFG chosen for this study), an herbicide with glyphosate as its sole active ingredient. This was chosen based on the similarity of extent of growth inhibition 
of various yeast strains to the majority of the CFG tested in conditions with and without supplementation of the aromatic amino acids (WYF) (Ravishankar, Pupo and Gallagher 2019).

Carrying out QTL analysis in minimal media supplemented with WYF (tryptophan, tyrosine and phenylalanine), allowed the analysis of the effects caused by not only the principal ingredient, glyphosate, but also the other inert additives in the commercial formulation of this herbicide. In the previous study (Ravishankar, Pupo, and Gallagher 2019), S288c was found to be sensitive to pure glyphosate but rescued on supplementing WYF. The addition of WYF when phenotyping, minimizes the glyphosate sensitivity and isolates the effects of the additives. The sensitivity of S288c to Cr41 treatment of the S288c x RM11 hybrid was narrowed down to two loci in minimal media and three loci in media supplemented with WYF (Fig 1A and B). Within these regions, mutants from the S288c knockout collection were screened to identify the loci likely responsible for the variation in growth (data not shown). The loci identified on $\mathrm{Cr} 41$ treatment in YM were on chromosomes 5 and 13, which were traced back to TIR1 (Fig 1C) and HFA1 (Fig 1D), respectively. On chromosome 13, HFA1 was the common locus mapped in both media conditions. The QTL analysis carried out in minimal media supplemented with WYF gave rise to two more loci on chromosomes 2 and 6 . The chromosome 2 region that was mapped contained two genes linked to Cr41 resistance, they were, $A A C 3$ (Fig 1F) and MUD1 (Fig 1E). The peak on chromosome 6 was found to be linked to PTR3.

The QTL analysis in minimal media was traced back to two candidate genes, one of which is a cell wall mannoprotein and the other is involved in mitochondrial fatty acid synthesis. Tir1 is a serine-alanine-rich cell wall mannoprotein (Marguet, Guo, and Lauquin 1988) that also 
belongs to a group of heme-repressed anaerobic genes (Cohen 2001; Sertil et al. 1997). Hfa1 is a $250 \mathrm{kDa}$ protein required for mitochondrial function. It is a specific acetyl-CoA carboxylase that plays a crucial role in the production of malonyl-CoA that serves as a substrate for intraorganellar fatty acid synthesis (Hoja et al. 2004).

The genetic linkage analysis of cells supplemented with WYF, also identified three other genes along with HFA1, i.e., AAC3, MUD1 and PTR3. AAC3, like HFA1, codes for a mitochondrial protein. Aac3 acts as an inner membrane ATP/ADP translocator (Kolarov et al. 1990), while MUD1 encodes a U1 snRNP, a protein that plays a key role in 5' splice site selection (Liao et al. 1993). The final candidate gene was PTR3, which is a component of the Ssy1p-Ptr3p-Ssy5p plasma membrane amino acid sensor system that sends signals regarding the amino acid levels in the immediate environment that help the cell in regulation (Forsberg et al. 2001).

\section{Testing effects of $\mathrm{Cr} 41$ on growth phenotype of haploid mutants}

Deletion mutants were used to evaluate the genes identified in the QTL analysis using a semi-quantitative growth assay. The BY4741 (an S288c derivative) knockout collection was used to test all the S288c knockout mutants within these intervals. Knockouts of the genes identified in the QTL analysis were made in RM11, the resistant strain. The RM11 and S288c mutants were tested on minimal media, media supplemented with WYF and media supplemented with aspartic acid (D) (Fig 2). The haploid cells were tested in the absence and presence of $0.15 \%$ Cr41 and $0.15 \%$ PG (pure glyphosate). In minimal media, all RM11 mutants were resistant to Cr41 as well as PG. To ensure that the resistance seen in RM11 mutants was not due to its natural ability to tolerate higher amounts of glyphosate, the assay was carried out with $0.275 \%$ 
Cr41 and PG for these strains, resulting in the same observation (Fig S1). Wild-type and all S288c mutants ( $h f a 1 \Delta, a a c 3 \Delta$, and tir $1 \Delta)$, with an exception of mud1 $1 \Delta$, were found to be sensitive to $\mathrm{Cr} 41$ but resistant to PG. This indicates that the sensitivity of S288c was specific to the additives in the commercial herbicide formulations and not specific to glyphosate alone. It was interesting to find that the deletion of MUD1 in S288c led to a resistant phenotype. S288c ptr3 $\triangle$ was not tested due to its synthetic lethality with LEU2 (Nigavekar and Cannon 2002;

Sadhu et al. 2018). S288c is auxotrophic for LEU2, preventing the knocking out of PTR3. Supplementing the media with WYF and D only led to a mild alleviation of growth inhibition in the S288c mutants, and not a rescue of the growth phenotype observed on $\mathrm{Cr} 41$ treatment. This indicates that, in the QTL analysis, the RM11 alleles of HFA1, AAC3, and TIR1 contribute to the tolerance of $\mathrm{Cr} 41$ treatment. The contribution of each of these genes could be attributed to the numerous SNPs between the S288c and RM11 alleles of these genes (Fig S2).

\section{Testing growth phenotype of double mutants of the QTL candidate genes provide better understanding of its role in $\mathrm{Cr} 41$ tolerance}

The mutants of all the QTL candidates tested in RM11, the resistant strain, continued to be resistant to $\mathrm{Cr} 41$. In order to gain a better understanding of the interactions in the pathways and genes involved in maintaining this resistant phenotype, double mutants of these candidates were exposed to $\mathrm{Cr} 41$ (Fig 3). In rich media, YPD the cells were grown in the presence and absence of $1.25 \% \mathrm{Cr} 41$; all of the mutant strains tested showed no change in $\mathrm{Cr} 41$ resistance, with an exception of the $h f a 1 \Delta$ tir1 $\Delta$ double mutant (Fig 3A). In minimal media, all the mutants tested were found to be sensitive (Fig 3B). On supplementing the media with WYF 
and aspartic acid, only aac3 $\Delta$ tir $1 \Delta$ was rescued (Fig 3C and D). Alleviation of growth inhibition was not seen in the case of aac3 $h f a 1 \Delta$ and $h f a 1 \Delta$ tir $1 \Delta$, which were found to be sensitive to Cr41 exposure.

\section{Transcriptome analysis reveals effects of $\mathrm{Cr} 41$ on carbon metabolism}

Transcriptome analysis was carried out to determine if the candidate genes from the QTL were differentially regulated on exposure to Cr41 (Ravishankar et al. 2019). Fructose and mannose metabolism were the only pathways up-regulated based on KEGG pathway analysis (Fig 4A). The KEGG pathway analysis showed that the pentose phosphate pathway, carbon metabolism and biosynthesis of secondary metabolites were significantly down-regulated (Fig 4B). The mitochondrial genes located within peaks in the QTL analysis of the S288c x RM11 segregants play a role in carbon metabolism. The pentose phosphate pathway has a crucial role in the generation of NADPH, providing the reducing power that is required during amino acid biosynthesis (Minard et al. 1998; Miosga and Zimmermann 1996). Ribose-5-phosphate is one of the main products of the pentose phosphate pathway and is required for the production of various secondary metabolites and amino acids such as tryptophan, tyrosine, and histidine (Kondo et al. 2004). The pentose phosphate pathway and glycolysis are coupled reactions that are a significant portion of metabolism in a cell. During glycolysis, glucose is converted into pyruvate which can then be converted into acetyl CoA in the mitochondria. The acetyl CoA can then be converted into citrates and other intermediate compounds that fuel the tricarboxylic acid cycle (TCA). Hence, the Cr41 influence on mitochondrial genes can further hinder the

process of carbon metabolism, and in turn, the production of many secondary metabolites. 


\section{Cr41-resistant ILE (in-lab evolution) strains showed duplication of multiple mitochondrial genes}

Saccharomyces cerevisiae shows great genetic diversity across the species (Song et al. 2015). Previously, using this diversity, four genetically diverse strains, two sensitive (YJM789 and S288c) and two resistant (AWRI1631 and RM11) strains were exposed to Cr41 for a prolonged period of time to determine the numerous routes they could take to attain resistance (Ravishankar et al. 2019). The strains were evolved for $~ 300$ generations, and Cr41 resistant yeast were identified. Single colonies of the resistant yeast were isolated, which were then sequenced. The whole genome sequencing data was analyzed to identify genes containing SNPS and CNV (copy number variation) with respect to $\mathrm{Cr} 41$ treatment. A recent study identified 901 proteins in the yeast cell as high-confidence mitochondrial proteins (Morgenstern et al. 2017). The ILE analysis identified that 259 genes either contained SNPs or underwent CNV. These genes were termed as "affected genes". Further analysis revealed that 32 of these 259 ILE genes encoded proteins that were a part of the mitochondrial proteome (Fig 5A). S288c contained modifications in 13 genes, which was the largest number of mitochondrial genes in comparison to all the other strains (Fig 5B). AWRI1631 contained four modified genes, of which DTD1 was the only unique gene; the other three were shared with YJM789. Dtd1 is a DTyrosine-tRNA deacylase that plays a role in the alteration of the cell's protein synthesis machinery (Soutourina et al. 2000). The three genes found in YJM789 ILEs were ACH1, PET9 and $A B F 2$. Ach1, similar to that of Hfa1, has CoA transferase activity, although it is mainly involved in the conversion of succinyl CoA to acetate (Buu et al. 2003). PET9 is the paralog of AAC3 which was one of the genes identified in the QTL analysis. Pet9 is the major ADP/ATP carrier of the 
mitochondrial inner membrane that imports cytosolic ADP into mitochondria in exchange for ATP (Chen 2004). Due to the genetic variation that lies across the species, strains use different routes to attain resistance to a certain stressor. In some cases, there could be similarity to a large extent with respect to the routes taken, as in the case of YJM789 and AWRI1631, while some strains can adapt a completely different route, such as S288c. In all the strains analyzed, numerous genes were duplicated in the Cr41 evolved strains; however, most of them were found to contain at least a few genes that encode mitochondrial proteins.

\section{Increased uptake of iron upon $\mathrm{Cr} 41$ exposure in the resistant strain}

Transcriptome analysis showed up-regulation of the metal transporters FIT2, FIT3, CUP1-1, and FRE5 in the resistant strain, RM11. Based on the functions of these genes, the cell concentrations of iron, zinc, calcium, magnesium, potassium and phosphorous were tested. The levels of metals within the cells treated with glyphosate varied between $\mathrm{Cr} 41$ and PG exposure

(Fig 6 and Fig S3). In Cr41-treated RM11, an increase in iron levels was observed (p-value=0.04)

(Fig 6A). Measuring these iron levels in a population of cells and not single cells or its subcellular compartments could explain the observed variation, if only subpopulations of heterogeneous cells are adapting to the stress in this manner. Due to the coupled uptake of iron and zinc, the levels of zinc were measured. Additionally, zinc increased ( $p$-value $=0.006)$ only in the case of PG exposure in RM11 (Fig 6). The presence of Fe-S clusters are critical for numerous functions within the cell acting as cofactors for enzymes involved in pathways such as the TCA cycle (Lill et al. 2015; Melber and Winge 2018). Fe-S intermediates originate in the mitochondria and move to the cytoplasm for assembly of Fe-S clusters (Pandey et al. 2019). Increased flux of iron 
by RM11, the resistant strain, likely attributes to an increase in Fe-S clusters needed to satisfy the requirement of cofactors in the various carbon metabolism processes.

\section{Discussion}

Commercial formulations of glyphosate contain glyphosate as their principal component and various additives that act to increase herbicide activity and application characteristics. Different herbicides use different adjuvants and surfactants in varying concentrations to enhance postemergence herbicide performance, increase absorption into plant tissue, act as a buffering and wetting agent, decrease photodegradation of the herbicide, etc. Although individually inert, these chemicals in unison, and in the presence of the active ingredient, have numerous effects on the functioning of cells (Ravishankar et al. 2019). Based on their genetic makeup, strains use varying routes to attain tolerance or resistance to these products.

Quantitative trait loci (QTL) analysis uses the underlying variation between two strains to identify loci that contribute to a specific phenotype. In this study the phenotype analyzed was sensitivity or resistance of cells exposed to $\mathrm{Cr} 41$ in terms of the growth phenotype. Growth inhibition of BY4741 (derived from S288c) on exposure to Cr41, can be slightly alleviated with supplementation of aromatic amino acids or aspartic acid, but a complete rescue is not observed. A complete rescue of BY4741 cells is observed upon supplementation of WYF and D when exposed to pure glyphosate. RM11 is a vineyard strain that does not show growth inhibition on exposure to Cr41. QTL analysis of this segregant collection on exposure to $\mathrm{Cr} 41$ 
identified loci that mainly established an underlying role of mitochondria in the maintenance of the cell's functionality on exposure to $\mathrm{Cr} 41$.

Mitochondria play a crucial role in the maintenance of numerous activities in a cell that range from ATP synthesis to metabolism of amino acids, lipids and heme (Galluzzi et al. 2012; Lill 2009; Shutt and McBride 2013). Although metabolism and ATP synthesis are the main functions of the mitochondria, it is lesser known for its danger signaling mechanism through retrograde response (Galluzzi et al. 2012; Parikh et al. 1987). When mitochondria are affected by a stress, they release a retrograde signal that results in the nuclear localization of Rtg1/3 complex (Sekito et al. 2000). This complex is responsible for the regulation of expression of various enzymes involved in mitochondrial metabolism, peroxisomal biogenesis, and stress response pathways (Chelstowska and Butow 1995). The activation of this complex depends on the phosphorylation of Rtg3, which is yet to be understood. RTG3 was down-regulated by 1.5 -fold in S288c cells exposed to Cr41. Low levels of ATP play an important role in the interaction of Rtg2 and Mks1, which may have resulted in the lack of activation of retrograde response in S288c cells, as a greater amount of the herbicide may be entering these cells. The RM11 allele of MKS1 was also identified in the QTL (data not shown), but was not included due to the high frequency of its appearance in numerous stress-induced phenotypes.

Fe-S clusters are inorganic cofactors for various enzymes, making them crucial for amino acid biosynthesis and the tricarboxylic acid cycle (Beinert et al. 1997). The primary role of Fe-S clusters is in the electron transport chain; however, they also function in sensing the environmental conditions and in turn, regulate gene expression (Waldron et al. 2009). The 
assembly of these Fe-S clusters mainly takes place in the mitochondria. Some of these Fe-S intermediates are then transported to the cytoplasm to serve as cofactors in diverse reactions. In cases of stress, the mitochondria are performing multiple functions to maintain homeostasis within the cell. To assemble sufficient levels of Fe-S clusters, cells such as RM11 import larger amounts of iron by upregulating iron transporters on exposure to $\mathrm{Cr} 41$.

A mitochondrial acetyl CoA carboxylase, $\mathrm{Hfa} 1$ is involved in the degradation of fatty acids to produce acetyl CoA, which is a reversible reaction in situations where de novo fatty acid synthesis is carried out (Kurat et al. 2006). The yeast plasma membrane is comprised of a lipid bilayer with various mannoproteins like Tir1, which control the entry and exit of environmental toxins such as herbicides. Hence, all the effects in fatty acid synthesis, ATP transport to the cytosol, etc. direct back to the importance of a functionally sound mitochondria to tolerate the effect of chemicals, such as commercial formulations of glyphosate. The involvement of mitochondria in the resistance of human cells to other formulations of glyphosate, such as RoundUp, have been shown in recent studies (Hao et al. 2019). With increasing evidence in higher eukaryotes, that toxicity of glyphosate-based herbicides is not limited to their active ingredient (Jacques et al. 2019). Subsequently, it is becoming more important to study the effects of these commercial formulations as a whole, with a goal of understanding their effect on different cellular components of the cell.

\section{Conclusion}


The genetic variation in herbicide resistance between the sensitive laboratory yeast strain S288c and resistant vineyard isolate RM11 was exploited by undertaking a QTL analysis and identifying loci contributing to resistance to Credit41, a commercial formulation containing glyphosate. Among the candidate genes identified, AAC3 and HFA1 were notable and highlighted the potential role of mitochondrial function in resistance to Credit41. Cells adapted to Credit41 exposure increased the copy number of PET9, a paralog of $A A C 3$, among numerous other mitochondrial proteins. The transcriptome analysis of Credit41-exposed cells also pointed out the involvement of mitochondria in resisting the effects of Credit41, as evidenced by upregulation of FIT2 and FIT3, which in turn increased the iron uptake crucial for Fe-S production. Changes across the genome and transcriptome in yeast cells exposed to Credit41 emphasize the need to thoroughly test the collaborative effects of active ingredients and additives in commercial formulations of glyphosate-based herbicides before use.

\section{Acknowledgements}

We are indebted to Amaury Pupo for his help and guidance with the data analysis. We thank the Kruglyak lab for the S288c x RM11 collection and Angela Lee for the yeast knockout collection. We also thank the Genomics Core facility for their services, that was supported by National Institutes of Health (WV-INBRE grant: P20 GM103434) and National Institutes of Health (NIGMS Grant: U54 GM-104942). This work was supported by National Science Foundation (grant number MCB-1614573) and WVU PSCoR. We also thank students from the Genomics Capstone (BIO320) at WVU who piloted the ILEs and Cell and Molecular Biology Lab (BIO311). 


\section{References}

Beinert, Helmut, Richard H. Holm, and Eckard Münck. 1997. "Iron-Sulfur Clusters: Nature's Modular, Multipurpose Structures." Science 277(5326): 653-59.

Bloom, Joshua S. et al. 2013. "Finding the Sources of Missing Heritability in a Yeast Cross." Nature.

Bloom, Joshua S., Kotenko, Iulia, Sadhu, Meru J., Treusch, Sebastian., Albert, Frank W., Kruglyak, Leonid. 2015. “Genetic Interactions Contribute Less than Additive Effects to Quantitative Trait Variation in Yeast." Nature Communications 6.

Buu, Leh Miauh, Yee Chun Chen, and Fang Jen S. Lee. 2003. "Functional Characterization and Localization of Acetyl-CoA Hydrolase, Ach1p, in Saccharomyces Cerevisiae." Journal of Biological Chemistry 278(19): 17203-9.

Chelstowska, A., and R. A. Butow. 1995. "RTG Genes in Yeast That Function in Communication between Mitochondria and the Nucleus Are Also Required for Expression of Genes Encoding Peroxisomal Proteins." Journal of Biological Chemistry 270(30): 18141-46.

Chen, Xin Jie. 2004. "Sal1p, a Calcium-Dependent Carrier Protein That Suppresses an Essential Cellular Function Associated with the Aac2 Isoform of ADP/ATP Translocase in Saccharomyces Cerevisiae." Genetics 167(2): 607-17.

Cohen, B. D. 2001. "Induction and Repression of DAN1 and the Family of Anaerobic Mannoprotein Genes in Saccharomyces Cerevisiae Occurs through a Complex Array of Regulatory Sites." Nucleic Acids Research 29(3): 799-808.

Forsberg, Hanna et al. 2001. "The Role of the Yeast Plasma Membrane SPS Nutrient Sensor in the Metabolic Response to Extracellular Amino Acids." Molecular Microbiology. 
Foury, Françoise, and Driss Talibi. 2001. "Mitochondrial Control of Iron Homeostasis. A Genome Wide Analysis of Gene Expression in a Yeast Frataxin-Deficient Strain." Journal of Biological Chemistry 276(11): 7762-68.

Galluzzi, Lorenzo, Oliver Kepp, and Guido Kroemer. 2012. “Mitochondria: Master Regulators of Danger Signalling." Nature Reviews Molecular Cell Biology 13(12): 780-88.

Hao, Youwu et al. 2019. "Roundup ${ }^{\circledR}$ Confers Cytotoxicity through DNA Damage and Mitochondria-Associated Apoptosis Induction." Environmental Pollution 252: 917-23.

Hoja, Ursula et al. 2004. "HFA1 Encoding an Organelle-Specific Acetyl-CoA Carboxylase Controls Mitochondrial Fatty Acid Synthesis in Saccharomyces Cerevisiae." Journal of Biological Chemistry 279(21): 21779-86.

Jacques, Mauricio Tavares et al. 2019. “Reprotoxicity of Glyphosate-Based Formulation in Caenorhabditis Elegans Is Not Due to the Active Ingredient Only." Environmental Pollution.

Kolarov, Jordan, Nadežda Kolarova, and Nathan Nelson. 1990. "A Third ADP/ATP Translocator Gene in Yeast." Journal of Biological Chemistry 265(21): 12711-16.

Kondo, Hiroki et al. 2004. "Pyridoxine Biosynthesis in Yeast: Participation of Ribose 5-Phosphate Ketol-Isomerase." Biochemical Journal 379(1): 65-70.

Kurat, Christoph F. et al. 2006. “Obese Yeast: Triglyceride Lipolysis Is Functionally Conserved from Mammals to Yeast." Journal of Biological Chemistry 281(1): 491-500.

Landry, Christian R., Julia Oh, Daniel L. Hartl, and Duccio Cavalieri. 2006. “Genome-Wide Scan Reveals That Genetic Variation for Transcriptional Plasticity in Yeast Is Biased towards MultiCopy and Dispensable Genes." Gene 366(2): 343-51.

Lee, Jonathan T., Alessandro L.V. Coradini, Amy Shen, and Ian M. Ehrenreich. 2019. "Layers of Cryptic Genetic Variation Underlie a Yeast Complex Trait." Genetics 211(4): 1469-82. 
Liao, X. C., J. Tang, and M. Rosbash. 1993. “An Enhancer Screen Identifies a Gene That Encodes the Yeast U1 SnRNP A Protein: Implications for SnRNP Protein Function in Pre-MRNA Splicing." Genes and Development 7(3): 419-28.

Lill, Roland. 2009. "Function and Biogenesis of Iron-Sulphur Proteins." Nature 460(7257): 83138.

Lill, Roland., Dutkiewicz, Rafal., Freibert, Sven A., Heidenreich, Torsten., Mascarenhas, Judita., Netz, Daili J., Paul, Viktoria D., Pierik, Antonio J., Richter, Nadine., Stümpfig, Martin., Srinivasan, Vasundara., Stehling, Oliver., Mühlenhoff, Ulrich. 2015. "The Role of Mitochondria and the CIA Machinery in the Maturation of Cytosolic and Nuclear Iron-Sulfur Proteins." European Journal of Cell Biology 94(7-9): 280-91. http://www.ncbi.nlm.nih.gov/pubmed/26099175 (September 1, 2019).

Marguet, D., X. J. Guo, and G. J.M. Lauquin. 1988. "Yeast Gene SRP1 (Serine-Rich Protein). Intragenic Repeat Structure and Identification of a Family of SRP1-Related DNA Sequences." Journal of Molecular Biology 202(3): 455-70.

Melber, Andrew, and Dennis R. Winge. 2018. "Steps Toward Understanding Mitochondrial Fe/S Cluster Biogenesis." In Methods in Enzymology, Academic Press Inc., 265-92.

Mesnage, R., N. Defarge, J. Spiroux de Vendômois, and G. E. Séralini. 2015. “Potential Toxic Effects of Glyphosate and Its Commercial Formulations below Regulatory Limits." Food and Chemical Toxicology 84: 133-53.

Minard, K I et al. 1998. "Sources of NADPH and Expression of Mammalian NADP+-Specific Isocitrate Dehydrogenases in Saccharomyces Cerevisiae." The Journal of biological chemistry 273(47): 31486-93. http://www.ncbi.nlm.nih.gov/pubmed/9813062 (September 1, 2019).

Miosga, T, and F K Zimmermann. 1996. "Cloning and Characterization of the First Two Genes of the Non-Oxidative Part of the Saccharomyces Cerevisiae Pentose-Phosphate Pathway." Current genetics 30(5): 404-9. http://www.ncbi.nlm.nih.gov/pubmed/8929392 (September 1, 2019). 
Morgenstern, Marcel et al. 2017. “Definition of a High-Confidence Mitochondrial Proteome at Quantitative Scale." Cell Reports 19(13): 2836-52.

Mortimer, Robert K., Patrizia Romano, Giovanna Suzzi, and Mario Polsinelli. 1994. “Genome Renewal: A New Phenomenon Revealed from a Genetic Study of 43 Strains of Saccharomyces Cerevisiae Derived from Natural Fermentation of Grape Musts." Yeast.

Mühlenhoff, Ulrich, and Roland Lill. 2000. "Biogenesis of Iron-Sulfur Proteins in Eukaryotes: A Novel Task of Mitochondria That Is Inherited from Bacteria." Biochimica et Biophysica Acta Bioenergetics 1459(2-3): 370-82.

Nigavekar, Shraddha S., and John F. Cannon. 2002. "Characterization of Genes That Are Synthetically Lethal with Ade3 or Leu2 in Saccharomyces Cerevisiae." Yeast 19(2): 115-22.

Pandey, Ashutosh K, Jayashree Pain, Andrew Dancis, and Debkumar Pain. 2019. “Mitochondria Export Iron-Sulfur and Sulfur Intermediates to the Cytoplasm for Iron-Sulfur Cluster Assembly and TRNA Thiolation in Yeast." Journal of Biological Chemistry 294(24): 9489-9502.

http://www.jbc.org/cgi/doi/10.1074/jbc.RA119.008600 (September 1, 2019).

Parikh, V S et al. 1987. "The Mitochondrial Genotype Can Influence Nuclear Gene Expression in Yeast." Science (New York, N.Y.) 235(4788): 576-80.

http://www.ncbi.nlm.nih.gov/pubmed/3027892 (September 9, 2019).

Parsons, Ainslie B, Ron Geyer, Timothy R Hughes, and Charles Boone. 2003. "Yeast Genomics and Proteomics in Drug Discovery and Target Validation." Progress in cell cycle research 5: 15966. http://www.ncbi.nlm.nih.gov/pubmed/14593709 (September 15, 2019).

Pupo, Amaury et al. 2019. "MCHM Acts as a Hydrotrope, Altering the Balance of Metals in Yeast." Biological Trace Element Research. http://link.springer.com/10.1007/s12011-01901850-z (August 27, 2019). 
Ravishankar, Apoorva, Amaury Pupo, and Jennifer E.G. Gallagher. 2019. “Thickening of the Cell Wall Increases the Resistance of S. Cerevisiae to Commercial Formulations of Glyphosate." bioRxiv: 760694.

Rong-Mullins, Xiaoqing et al. 2017. "Genetic Variation in Dip5, an Amino Acid Permease, and Pdr5, a Multiple Drug Transporter, Regulates Glyphosate Resistance in S. Cerevisiae." PLoS ONE 12(11).

Sadhu, Meru J. et al. 2018. "Highly Parallel Genome Variant Engineering with CRISPR-Cas9." Nature Genetics 50(4): 510-14.

dos Santos, Sandra C., Miguel Cacho Teixeira, Tânia R. Cabrito, and Isabel Sá-Correia. 2012. "Yeast Toxicogenomics: Genome-Wide Responses to Chemical Stresses with Impact in Environmental Health, Pharmacology, and Biotechnology." Frontiers in Genetics 3(APR).

Sekito, Takayuki, Janet Thornton, and Ronald A. Butow. 2000. "Mitochondria-to-Nuclear Signaling Is Regulated by the Subcellular Localization of the Transcription Factors Rtg1p and Rtg3p." Molecular Biology of the Cell 11(6): 2103-15.

Sertil, O, B D Cohen, K J Davies, and C V Lowry. 1997. "The DAN1 Gene of S. Cerevisiae Is Regulated in Parallel with the Hypoxic Genes, but by a Different Mechanism." Gene 192(2): 199-205. http://www.ncbi.nlm.nih.gov/pubmed/9224891 (September 1, 2019).

Shutt, Timothy E, and Heidi M McBride. 2013. "Staying Cool in Difficult Times: Mitochondrial Dynamics, Quality Control and the Stress Response." Biochimica et biophysica acta 1833(2): 417-24. http://www.ncbi.nlm.nih.gov/pubmed/22683990 (September 9, 2019).

Smith, Erin N., and Leonid Kruglyak. 2008. "Gene-Environment Interaction in Yeast Gene Expression." PLoS Biology 6(4): 810-24.

Song, Giltae et al. 2015. "AGAPE (Automated Genome Analysis PipelinE) for Pan-Genome Analysis of Saccharomyces Cerevisiae." PloS one 10(3): e0120671. 
Soutourina, Julie, Sylvain Blanquet, and Pierre Plateau. 2000. “D-Tyrosyl-TRNA(Tyr) Metabolism in Saccharomyces Cerevisiae." Journal of Biological Chemistry 275(16): 11626-30.

Waldron, Kevin J., Julian C. Rutherford, Dianne Ford, and Nigel J. Robinson. 2009.

“Metalloproteins and Metal Sensing." Nature 460(7257): 823-30. 
Table 1: Mitochondrial proteome genes that underwent CNV

\begin{tabular}{|c|c|c|c|}
\hline S288c & YJM789 & AWRI1631 & RM11 \\
\hline RSM10 & ACH1 & ACH1 & no CNVs \\
\hline VMS1 & PET9 & PET9 & \\
\hline TP11 & ABF2 & ABF2 & \\
\hline TGL2 & & DTD1 & \\
\hline RRG1 & & & \\
\hline FMP16 & & & \\
\hline SED1 & & & \\
\hline PET100 & & & \\
\hline MRX14 & & & \\
\hline MRPL1 & & & \\
\hline COX26 & & & \\
\hline TRM1 & & & \\
\hline ECM18 & & & \\
\hline
\end{tabular}


A.

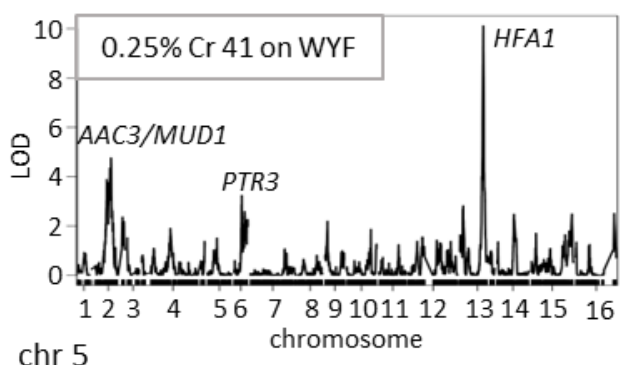

C. $\underline{\operatorname{chr} 5}$

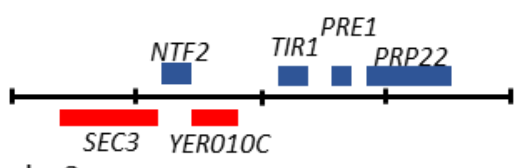

E. $\underline{\operatorname{chr} 2}$

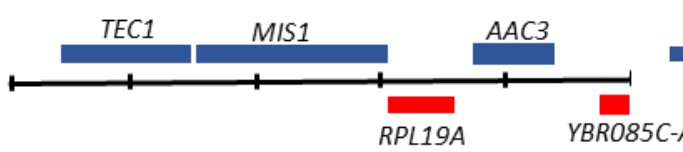

B.

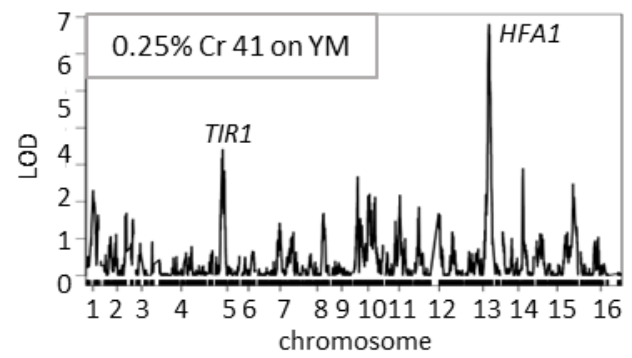

D. $\underline{\operatorname{chr} 13}$

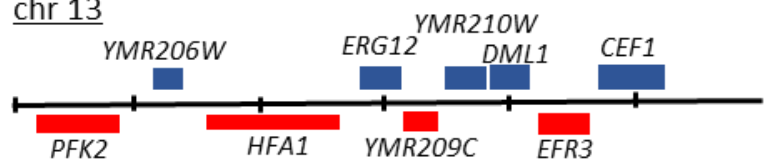

F. $\underline{\operatorname{chr} 2}$

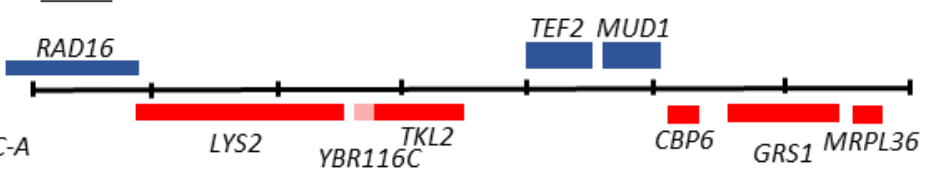

Fig 1: Genetic linkage analysis of glyphosate (Credit41) sensitivity in minimal media A. Genetic linkage of sensitivity of S288c to $0.25 \%$ Credit41 in YM+ WYF. LOD score (y-axis) was mapped across the yeast genome ( $\mathrm{x}$-axis) with chromosomes numbered left to right. B. Genetic linkage of sensitivity of $\mathrm{S} 288 \mathrm{c}$ to $0.25 \%$ glyphosate in YM. LOD score ( $y$-axis) was mapped across the yeast genome (x-axis) with chromosomes numbered left to right. C-F. The genomic loci under each peak identified in the QTL. Genes encoded on the top and bottom strands are in blue and red respectively on the following chromosomes. 
A.

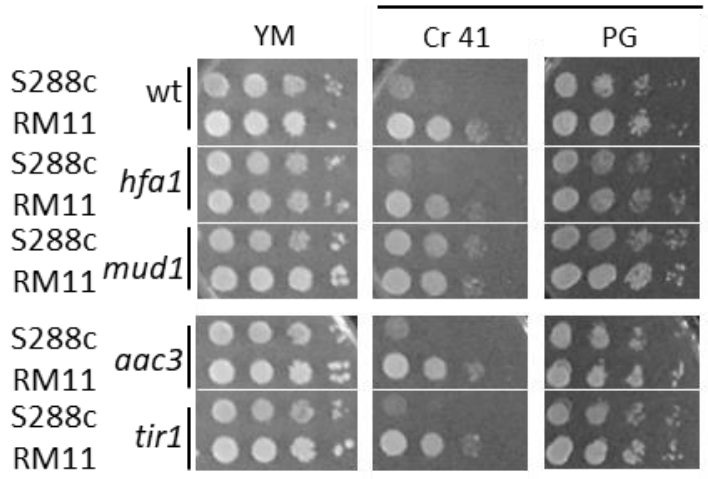

B.

RM11

WYF

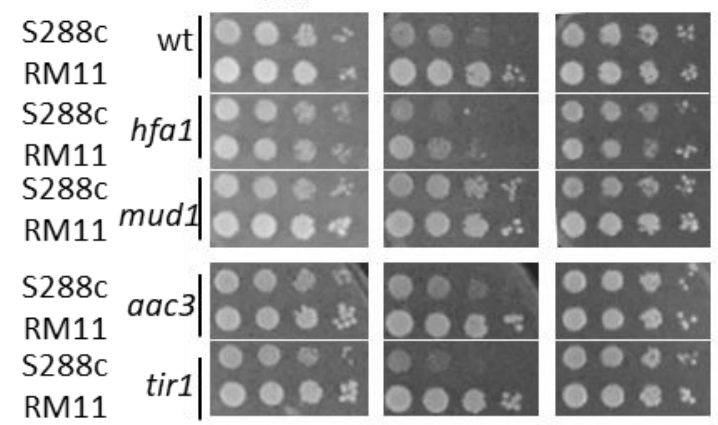

C.

RM11

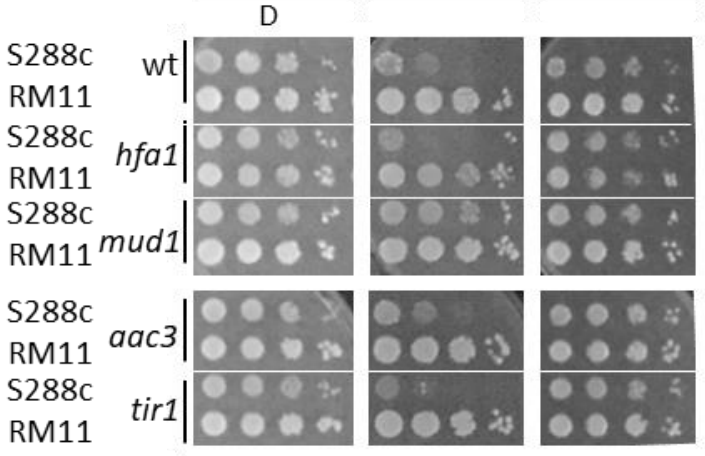

Figure 2: Growth of RM11 and S288c (BY4742) haploid mutants on exposure to Credit 41 (Cr41). Serial dilutions of RM11 and S288c wt, hfa1, mud1, aac3 and tir1 were grown on the following media in the presence and absence of $\mathrm{Cr} 41$ and pure glyphosate (PG). A. Minimal media with $0.15 \%$ glyphosate B. YM supplemented with aromatic amino acids with $0.15 \%$ glyphosate C. YM with aspartic acid with $0.15 \%$ glyphosate. 


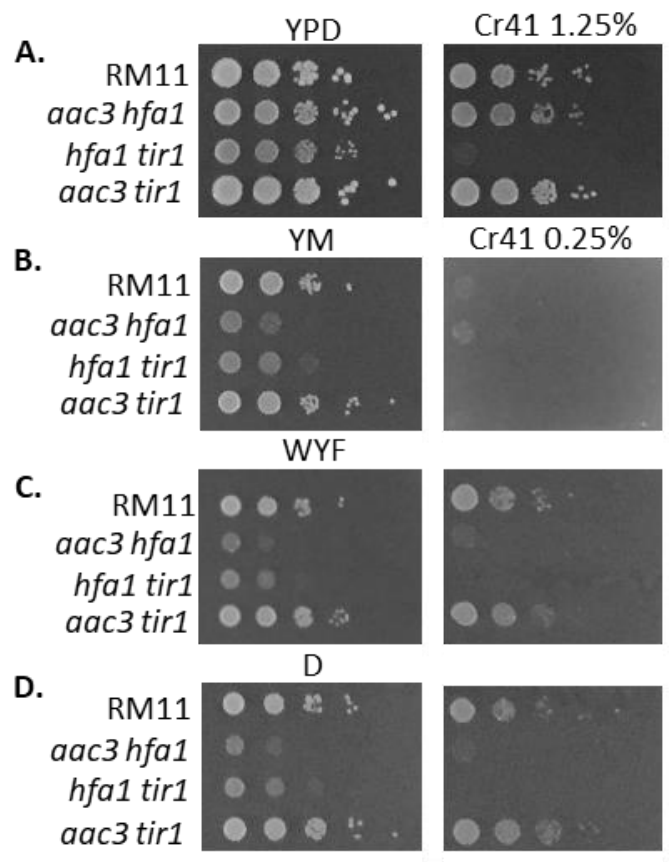

Figure 3: Growth of RM11 double knockouts on exposure to Credit 41 (Cr41). Serial dilutions of RM11 wt, aac3 hfa1, hfa1 tir1, and aac3 tir1 were grown on the following media in the presence and absence of Credit 41. A. Rich media, YPD with 1.25\% Cr41. B. Minimal media, YM with $0.25 \% \mathrm{Cr} 41$. C. YM with aromatic amino acids i.e., tryptophan (W), tyrosine (Y), phenylalanine (F) with $0.25 \% \mathrm{Cr} 41$. D. YM with aspartic acid (D) with $0.25 \% \mathrm{Cr} 41$. 

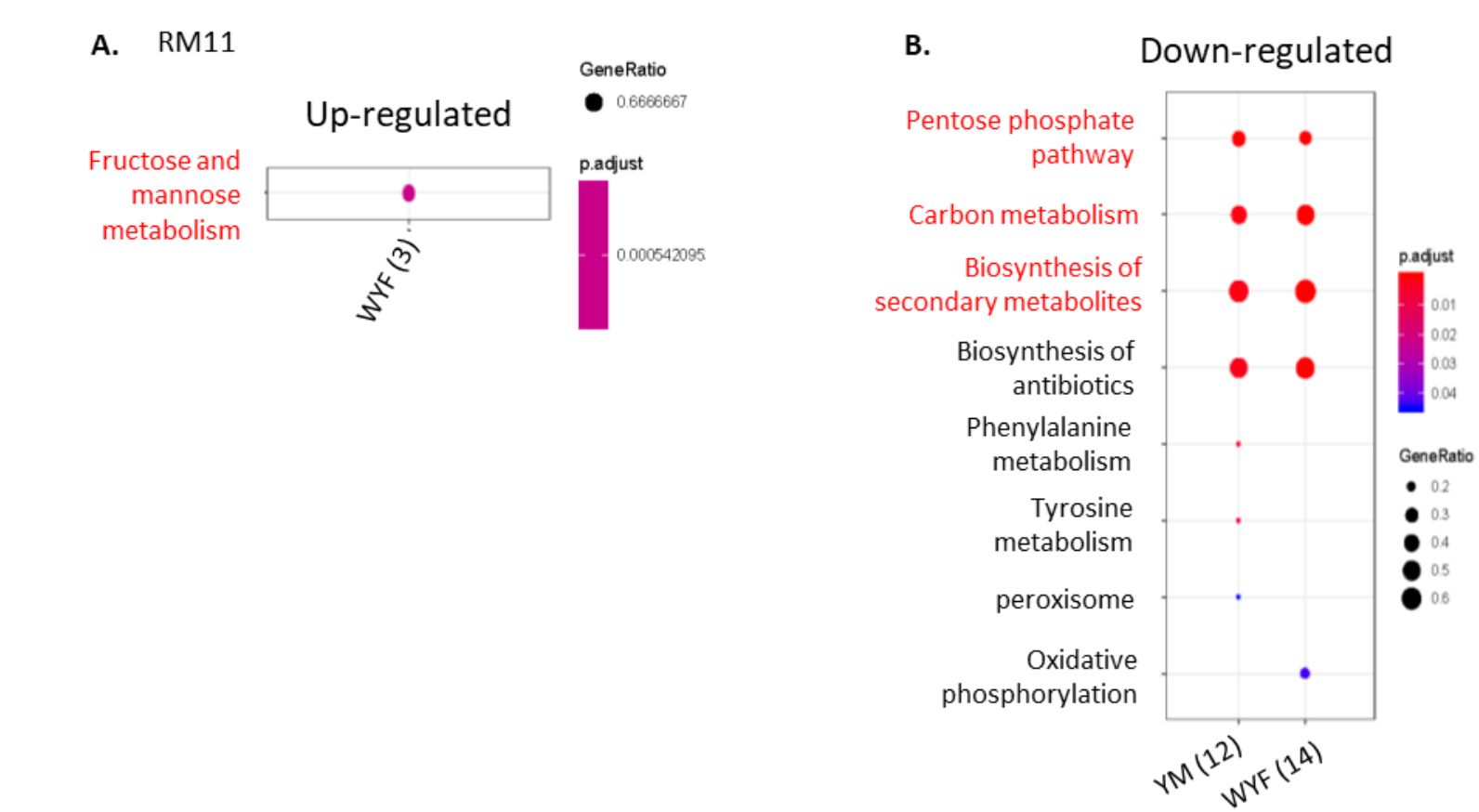

Fig 4: Gene expression analysis of RM11 on exposure to glyphosate (Credit41) in minimal media A. KEGG Pathway Enrichment analysis of up-regulated genes, identifying the different pathways involved and their extent of involvement. B. KEGG Pathway Enrichment analysis of down-regulated genes, identifying the different pathways involved and their extent of involvement 
A.

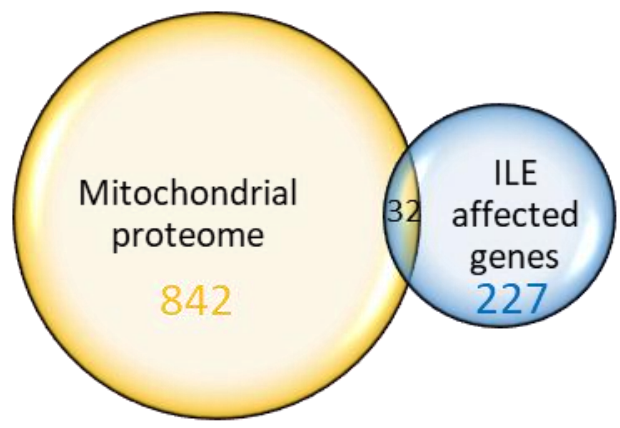

B.

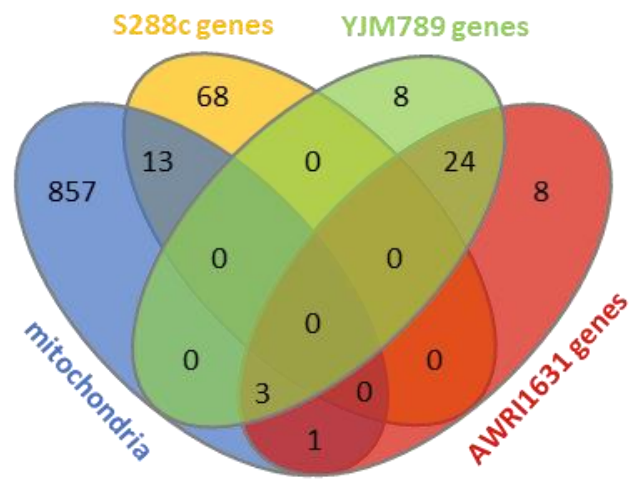

Fig 5: Analysis of genes affected in the in-lab evolutions and their relatedness to the mitochondria A. Venn-diagram representing genes common between the mitochondrial proteome and genes in the in-lab evolutions containing CNVs or SNPs. B. Venn-diagram representing commonalities between genes that underwent CNV in S288c, YJM789, AWRI1631 and the mitochondrial proteome 
A.

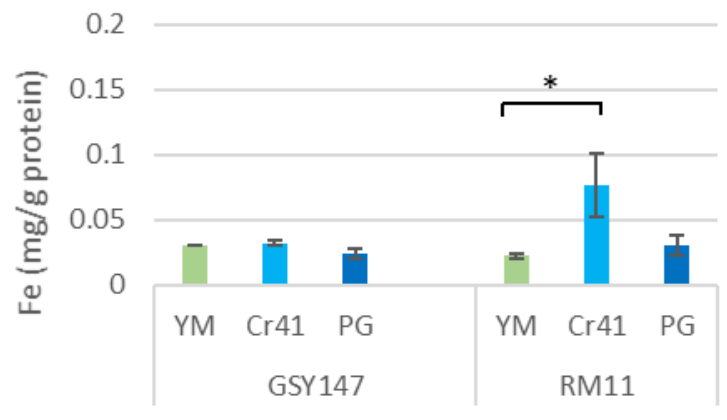

B.

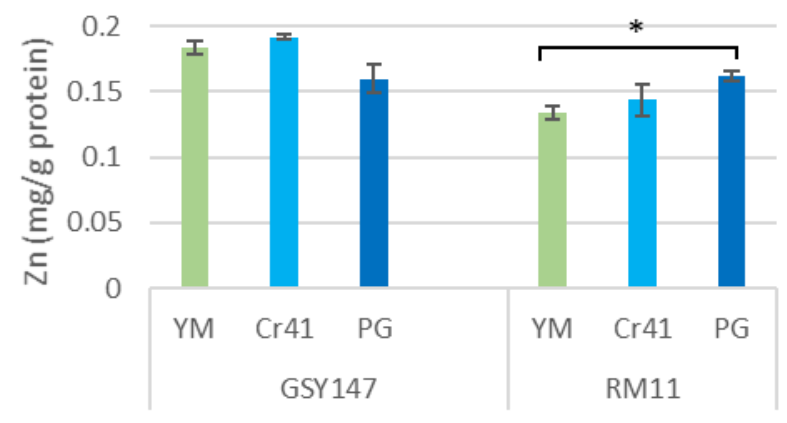

Fig 6: Change in metal amounts on glyphosate exposure. Concentration of metals was measured within the cells grown in minimal media with exposure to Credit41 ( $\mathrm{Cr} 41)$ or pure glyphosate (PG) for 90 minutes using ICP (Inductively Coupled Plasma) analysis. A. Levels of Iron within the cell. B. Levels of Zinc within the cells. 


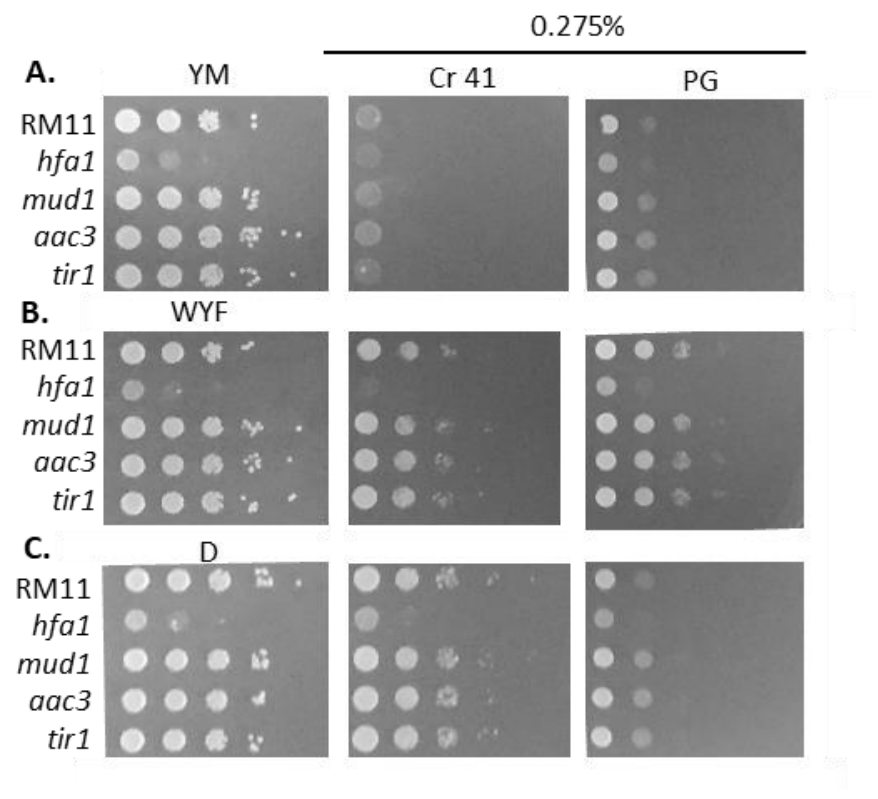

Figure S1: Growth of RM11 knockouts on exposure to Credit $41(\mathrm{Cr} 41)$ and pure glyphosate (PG). Serial dilutions of RM11, hfa1, mud1, aac3, and tir1 were grown on the following media in the presence of Credit41 and pure glyphosate A. YM with $0.275 \%$ Credit41 and pure glyphosate B. YM + aromatic amino acids with $0.275 \%$ Credit41 and pure glyphosate C. YM + aspartic acid with $0.275 \%$ Credit41 and pure glyphosate 


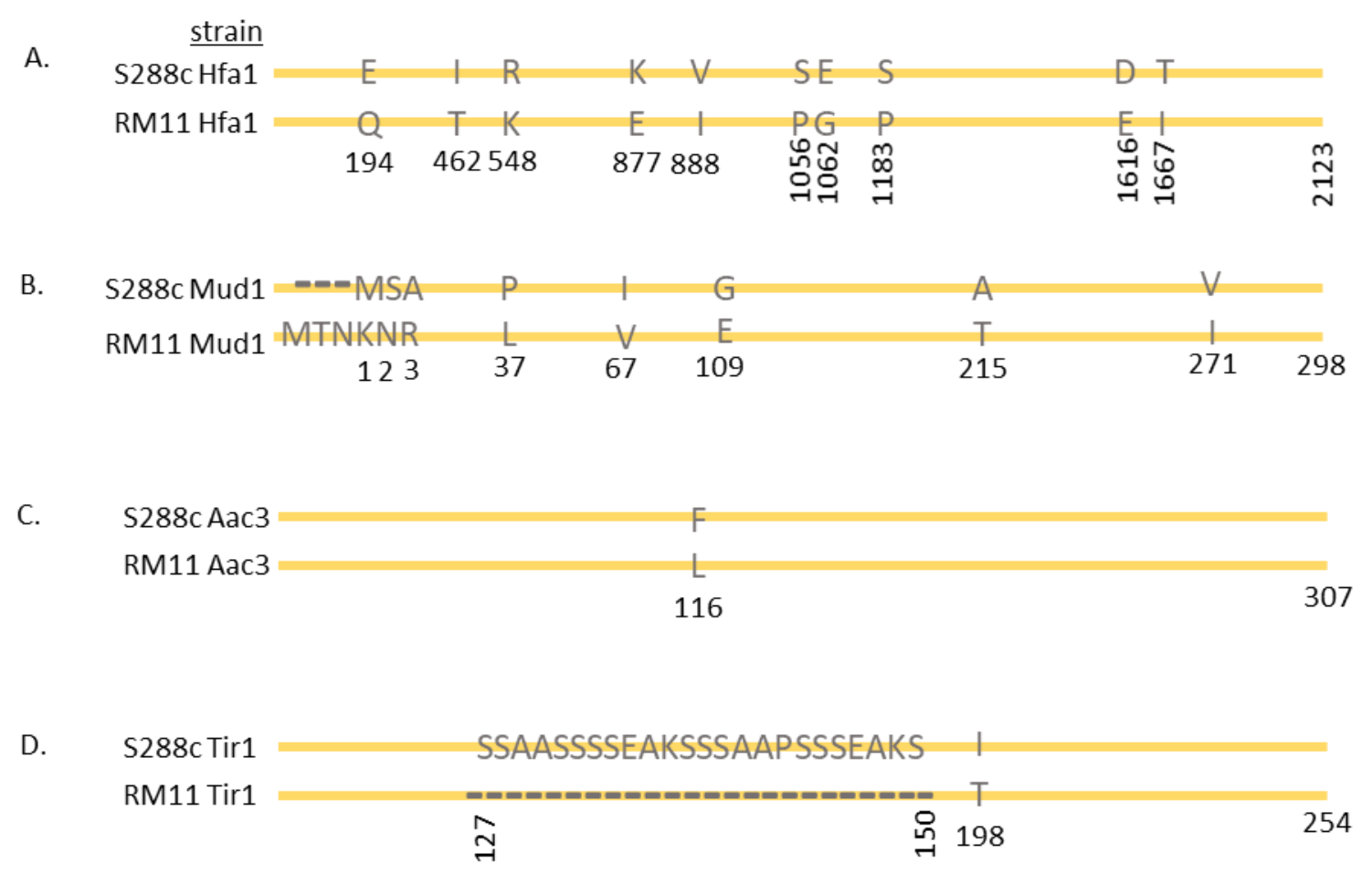

Figure S2: Protein alignment of QTL candidate genes. An alignment of the protein sequences of the genes identified in the QTL were carried out between S288c and RM11. The alignment highlights mutations in the amino acid sequence in grey. The hyphens represent a deletion of that amino acid. 

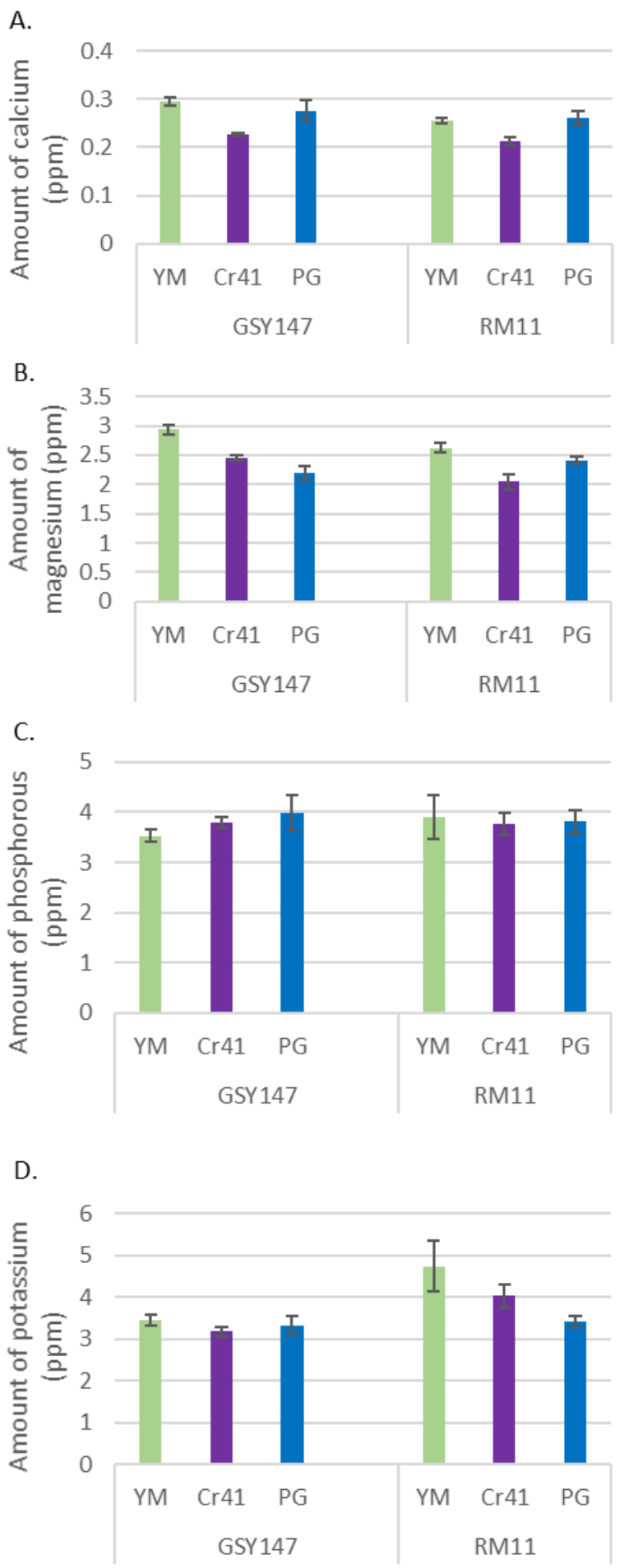

Figure S3: Change in concentrations of metals on glyphosate exposure. Concentration of metals was measured within the cells grown in minimal media with exposure to Credit41 (Cr41) or pure glyphosate (PG) for 90 minutes using ICP (Inductively Coupled Plasma) analysis. A. Levels of calcium within the cell B. magnesium C. phosphorous D. potassium 


\section{CHAPTER 6: CONCLUSION}

Since the introduction of RoundUp by Monsanto in 1970s, numerous commercial formulations of glyphosate-based herbicides (CFG) have been introduced. As reviewed in Chapter 2, all these formulations have glyphosate as their active ingredient, but with varying adjuvants and surfactants as additives. Although these additives were labeled inert based on individual testing, it has been shown that they can have a synergistic effect. As the mixtures of additives and their respective concentration is proprietary information, the manufacturers are not obligated to provide any further information on its components. This makes it tedious to decipher and estimate all their effects at the cellular level.

The aromatic amino acid biosynthetic pathway, commonly known as the shikimate pathway was the sole, known target of glyphosate. Chapter 3 showed that variation within $A R O 1$, the yeast ortholog of EPSPS (5-enolpyruvylshikimate-3-phosphate synthase), was not entirely responsible for resistance to a commercial formulation of glyphosate, Credit41. A QTL analysis revealed the contribution of a pleiotropic drug marker such as Pdr5, as well as, an amino acid permease, Dip5, in displaying a resistant phenotype on exposure to Credit41. Dip5 is mainly responsible for the import of aspartic aid and glutamic acid into the cell. The structural similarity between aspartic and glutamic acid with glyphosate, and the down-regulation of Dip5 either by mutation or with supplementation of excess aspartic acid, point to Dip5 as the transporter of glyphosate into the cell. Among the tested strains, some alleles of Dip5 imported higher amounts of glyphosate into the cell, making the strain more sensitive. Pdr5 was one of the pleiotropic drug markers that was involved in the active pumping out of glyphosate from 
the cell. Hence, in this chapter we identified two crucial routes of import and export of glyphosate on exposure to Credit41.

To identify the targets of the CFG that lie outside the shikimate pathway, further study was carried out in agricultural isolates that had been exposed to glyphosate-based herbicides prior to isolation. The first step was to establish if all the formulations had similar effects on the growth phenotype of different yeast strains. Chapter 4 comprised a growth assay showing variation among different formulations; hence, a formulation that affected cells in a manner similar to a subset of other formulations, was chosen for the rest of the study. Genes that were differentially expressed in sensitive and resistant strains, to combat the effects of stress, specifically in response to Credit41 exposure, were identified. The variations at the genome level were also deciphered in cells that were evolved in media containing Credit41. Both studies highlighted the involvement of the cell wall, with respect to Credit41-resistance. The addition of calcofluor white, which thickens cells walls, showed that the cell wall, specifically Sed1, significantly contributed to the resistance of the cell to Credit41.

In Chapter 5, a QTL analysis was carried out between a sensitive, laboratory strain and a resistant, vineyard strain. The loci contributing to resistance, contained candidate genes such as AAC3 and HFA1 among others. These genes illustrated the involvement of mitochondrial proteins in response and resistance mechanisms pertaining to Credit41-tolerance. The whole genome sequencing data showed an increase in copy number of PET9, an AAC3 paralog, among other mitochondrial genes, making it evident that mitochondrial function was intertwined with Credit41 resistance. On parsing the previously carried out gene-expression analysis, an upregulation of genes involved in iron-uptake, such as FIT2 and FIT3 were found in the resistant 
strains. The mitochondria play a vital role in metabolism of secondary metabolites and Fe-S production, both of which could indirectly aid in Credit41 resistance. The mitochondria are functionally ubiquitous, in terms of its interdependence with so many other pathways. Dysfunctional mitochondria impact the cells ability to resist environmental stressors such as exposure to Credit41.

The studies carried out in the past few decades have been instrumental in shedding light on the health implications that these commercial formulations may have (Folmar et al. 1979; Silva et al. 2002). As the concept of synergistic effects of herbicides came to light, more studies were carried out with commercial formulations to test these effects on multiple pathways and cell types. Some of the effects found were damage to the rat liver and kidneys (Olorunsogo et al. 1979; Jayasumana et al. 2014), disruption of endocrine-signaling pathways (Gasnier et al. 2009; Romano et al. 2012; Thongprakaisang et al. 2013), a link to non-Hodgkin's lymphoma , and even alteration of the digestive microbiome (Shehata et al. 2013; Krüger et al. 2014). Two of the most controversial reports in the recent past were that of the US EPA, stating glyphosate is not carcinogenic to humans, and that of the International Agency for Research on Cancer (IARC), declaring that it probably is carcinogenic to humans. The main reason for this discrepancy is that the EPA mainly relied on unpublished regulatory studies that were mostly negative and used data from studies carried out on technical glyphosate; however, IARC relied primarily peer-reviewed studies that were $70 \%$ positive. The studies considered by the IARC did not focus on pure glyphosate, but on glyphosate-based herbicides and AMPA assays (Benbrook 2019). To regulate the additives and their concentrations, to an extent such that it is safe for 
humans and animals, extensive research is an absolute requirement to decipher the exact components of these herbicides and their biological fate and consequences. 


\section{References}

Folmar, L. C., H. O. Sanders, and A. M. Julin, 1979 Toxicity of the herbicide glyphosate and several of its formulations to fish and aquatic invertebrates. Arch. Environ. Contam. Toxicol.

Gasnier, C., C. Dumont, N. Benachour, E. Clair, M. C. Chagnon et al., 2009 Glyphosate-based herbicides are toxic and endocrine disruptors in human cell lines. Toxicology 262: 184-191.

Guyton, K. Z., D. Loomis, Y. Grosse, F. El Ghissassi, L. Benbrahim-Tallaa et al., 2015 Carcinogenicity of tetrachlorvinphos, parathion, malathion, diazinon, and glyphosate. Lancet Oncol. 16: 490-491.

Jayasumana, C., S. Gunatilake, and P. Senanayake, 2014 Glyphosate, hard water and nephrotoxic metals: Are they the culprits behind the epidemic of chronic kidney disease of unknown etiology in Sri Lanka? Int. J. Environ. Res. Public Health 11: 2125-2147.

Krüger, M., W. Schrödl, and I. Pedersen, 2014 Detection of Glyphosate in Malformed Piglets. J. Environ. Anal. Toxicol.

Myers, J. P., M. N. Antoniou, B. Blumberg, L. Carroll, T. Colborn et al., 2016 Concerns over use of glyphosate-based herbicides and risks associated with exposures: A consensus statement. Environ. Heal. A Glob. Access Sci. Source 15:.

Olorunsogo, O. O., E. A. Bababunmi, and O. Bassir, 1979 Effect of glyphosate on rat liver mitochondria in vivo. Bull. Environ. Contam. Toxicol. 22: 357-364.

Romano, M. A., P. Wisniewski, P. Viau, R. M. Romano, D. A. Campos et al., 2012 Glyphosate impairs male offspring reproductive development by disrupting gonadotropin expression. Arch. Toxicol. 86: 663-673.

Shehata, A. A., W. Schrödl, A. A. Aldin, H. M. Hafez, and M. Krüger, 2013 The effect of glyphosate on potential pathogens and beneficial members of poultry microbiota in vitro. Curr. Microbiol. 66: 350-358.

Silva, E., N. Rajapakse, and A. Kortenkamp, 2002 Something from "nothing" - Eight weak 
estrogenic chemicals combined at concentrations below NOECs produce significant mixture effects. Environ. Sci. Technol. 36: 1751-1756.

Thongprakaisang, S., A. Thiantanawat, N. Rangkadilok, T. Suriyo, and J. Satayavivad, 2013 Glyphosate induces human breast cancer cells growth via estrogen receptors. Food Chem. Toxicol. 59: 129-136. 